\title{
Weak solutions for unidirectional gradient flows: existence, uniqueness, and convergence of time discretization schemes
}

\author{
Masato Kimura and Matteo Negriø
}

\begin{abstract}
We consider the gradient flow of a quadratic non-autonomous energy under monotonicity constraints. First, we provide a notion of weak solution, inspired by the theory of curves of maximal slope, and then we prove existence (employing time-discrete schemes with different implementations of the constraint), uniqueness, power and energy identity, comparison principle and continuous dependence. As a by-product, we show that the energy identity gives a selection criterion for the (nonunique) evolutions obtained by other notions of solutions. Finally, we show that for autonomous energies the evolution obtained with the monotonicity constraint actually coincides with the evolution obtained by replacing the constraint with a fixed obstacle, given by the initial datum.
\end{abstract}

Mathematics Subject Classification. Primary 35K86, Secondary 49 J40.

Keywords. Gradient flow, Unilateral constraint.

\section{Introduction}

There are many physical phenomena, including fatigue, damage, and fracture of materials, see e.g. $[4,8,20-22,26]$ which are irreversible and grow in one direction following an energy gradient structure; modeling and analysis of such phenomena by nonlinear differential equations is a challenging and interesting problem. The following prototype problem is often considered as a mathematical model of such unidirectional gradient systems

$$
\left\{\begin{array}{l}
\dot{u}(t, x)=[\Delta u(t, x)+f(t, x)]_{+} \quad(t, x) \in(0, T) \times \Omega \\
u(0)=u_{0}
\end{array}\right.
$$

This work was supported by: Japan Society for the Promotion of Science (JP20KK0058), Japan Society for the Promotion of Science (JP20H01812), INdAM Istituto Nazionale di Alta Matematica. 
where $\dot{u}:=\partial_{t} u$ and $\Delta u$ is the Laplacian. The set $\Omega$ is a bounded domain in $\mathbb{R}^{d}$ and $[\cdot]_{+}$denotes the positive part, i.e., $[a]_{+}=\max \{a, 0\}$ for $a \in \mathbb{R}$. This is important as a model that features both unidirectional growth and energy gradient structure. The former is given by the positive part, which enforces monotonicity of $u$ in time; as far as the latter, note that the term $-\Delta u(t, x)-f(t, x)$ is the $L^{2}$-gradient of the energy

$$
\int_{\Omega} \frac{1}{2}|\nabla u(t, x)|^{2}-f(t, x) u(t, x) d x,
$$

under homogeneous Dirichlet or Neumann boundary condition. Note also that (1.1) is a dissipative system, since

$$
\begin{aligned}
\frac{d}{d t} & \left(\int_{\Omega} \frac{1}{2}|\nabla u(t, x)|^{2}-f(t, x) u(t, x) d x\right) \\
& =\int_{\Omega} \nabla u(t, x) \cdot \nabla \dot{u}(t, x)-f(t, x) \dot{u}(t, x)-\dot{f}(t, x) u(t, x) d x \\
& =-\int_{\Omega} \dot{u}(t, x)(\Delta u(t, x)+f(t, x)) d x-\int_{\Omega} \dot{f}(t, x) u(t, x) d x \\
& =-\int_{\Omega}|\dot{u}(t, x)|^{2} d x-\int_{\Omega} \dot{f}(t, x) u(t, x) d x ;
\end{aligned}
$$

the first term is the dissipation while the second plays the role of the power of external forces (see also Remark 2.9).

Due to the strong nonlinearity of (1.1), induced by the positive part, care must be taken in its mathematical handling. For instance, using the monotone graph $\alpha^{-1}$ with $\alpha(s):=[s]_{+}$, the parabolic equation in (1.1) can be written in the form of the doubly nonlinear evolution equation

$$
\alpha^{-1}(\dot{u}(t, x)) \ni \Delta u(t, x)+f(t, x) .
$$

However, in this case, $\alpha^{-1}$ is multivalued and its derivative is unbounded, so the standard theory of doubly nonlinear evolution equation [9] cannot be applied. Still, this theory is the technical background of the first existence and uniqueness result for (1.1), obtained in [7] assuming that $\Delta u_{0} \in L^{2}$ and $f \in A C\left(0, T ; L^{2}\right)$. An alternative proof of existence and uniqueness, based on minimizing movements, follows as a by product of the general results of [18] assuming $f \in A C\left(0, T ; L^{2}\right)$ and $u_{0} \in H^{1}$ (the original result [18] is actually stated for $f$ independent of time). More recently, in [2], the authors succeeded in directly constructing a strong solution, using a backward Euler scheme and assuming that $u_{0} \in H^{2}$, that $f \in L^{2}\left(0, T ; L^{2}\right)$, and that there exists $f^{\star} \in L^{2}$ satisfying $f(t, x) \leq f^{*}(x)$ for a.e. $(t, x) \in(0, T) \times \Omega$.

Our main goal is to give a new definition of weak solution for (1.1) and to show existence and uniqueness of the solution, assuming only $f \in L^{2}\left(0, T ; L^{2}\right)$ and $u_{0} \in H^{1}$. Our approach relies on the constrained gradient structure of (1.1). We employ techniques from the theory of curves of maximal slope $[5,13$, $14,24]$ which gives a new definition of weak solutions for gradient flows based on the energy (or power) inequality and on the notion of slope. 
More precisely, we consider an energy $\mathcal{F}:[0, T] \times H_{0}^{1} \rightarrow \mathbb{R}$ of the form

$$
\mathcal{F}(t, u)=\frac{1}{2} a(u, u)-\langle f(t), u\rangle,
$$

where $a(\cdot, \cdot)$ is a coercive, continuous bi-linear form in $H_{0}^{1}, f$ belongs to $L^{2}\left(0, T ; L^{2}\right)$, and $\langle\cdot, \cdot\rangle$ denotes the $L^{2}$-scalar product. Introducing the operator $A: H_{0}^{1} \rightarrow H^{-1}$ defined by $a(u, v)=-(A u, v)$ the Fréchet differential of $\mathcal{F}(t, \cdot)$ clearly reads

$$
d \mathcal{F}(t, u)[v]=a(u, v)-\langle f(t), v\rangle=-(A u+f(t), v),
$$

where $(\cdot, \cdot)$ denotes the duality between $H_{0}^{1}$ and $H^{-1}$. If $A u \in L^{2}$ we can write

$$
-d \mathcal{F}(t, u)[v]=\langle A u+f(t), v\rangle=\left\langle-\nabla_{L^{2}} \mathcal{F}(t, u), v\right\rangle .
$$

In particular, the positive part $[A u(t)+f(t)]_{+}$turns out to be the $L^{2}$-projection of $-\nabla_{L^{2}} \mathcal{F}(t, u)$ on the cone of positive functions. Therefore, a natural formulation for the unilateral gradient flow is

$$
\left\{\begin{array}{l}
\dot{u}(t)=[A u(t)+f(t)]_{+} \\
u(0)=u_{0} .
\end{array}\right.
$$

Technically, if $u(t) \notin H^{2}$ the above PDE makes sense whenever $A u(t)+f(t)$ is a locally finite Radon measure and $[A u(t)+f(t)]_{+}$is its positive part in the sense of Hahn decomposition (see Proposition 2.5).

Before proceeding with the content of our work, we provide, for the reader's convenience, an introductory presentation of unconstrained $L^{2}$-gradient flows in the framework of curves of maximal slope [5]. For sake of simplicity, we assume that $f \in A C\left(0, T ; L^{2}\right)$. Let $u \in H^{1}\left(0, T ; L^{2}\right)$ with $A u \in L^{2}\left(0, T ; L^{2}\right)$, then $u$ solves the parabolic problem

$$
\dot{u}(t)=A u(t)+f(t) \text { a.e. in }(0, T),
$$

if and only if $t \mapsto \mathcal{F}(t, u(t))$ is absolutely continuous and

$$
\dot{\mathcal{F}}(t, u(t)) \leq-\frac{1}{2}\|\dot{u}(t)\|_{L^{2}}^{2}-\frac{1}{2}\left\|\nabla_{L^{2}} \mathcal{F}(t, u(t))\right\|_{L^{2}}^{2}-\langle\dot{f}(t), u(t)\rangle,
$$

a.e. in $(0, T)$. Let us give a short proof of the equivalence between (1.3) and (1.4). If $\dot{u}(t)=-\nabla_{L^{2}} \mathcal{F}(t, u(t))$ a.e. in $(0, T)$ by [10, Lemma 3.3] the energy $t \mapsto \mathcal{F}(t, u(t))$ is absolutely continuous and by the chain rule

$$
\dot{\mathcal{F}}(t, u(t))=\left\langle\nabla_{L^{2}} \mathcal{F}(t, u(t)), \dot{u}(t)\right\rangle-\langle\dot{f}(t), u(t)\rangle \text { a.e. in }(0, T) .
$$

As $\dot{u}(t)=-\nabla_{L^{2}} \mathcal{F}(t, u(t))$ we have

$$
\left\langle\nabla_{L^{2}} \mathcal{F}(t, u(t)), \dot{u}(t)\right\rangle=-\frac{1}{2}\|\dot{u}(t)\|_{L^{2}}^{2}-\frac{1}{2}\left\|\nabla_{L^{2}} \mathcal{F}(t, u(t))\right\|_{L^{2}}^{2}
$$

and (1.4) follows. Conversely, by the chain rule and by Cauchy inequality

$$
\begin{aligned}
\dot{\mathcal{F}}(t, u(t)) & =\left\langle\nabla_{L^{2}} \mathcal{F}(t, u(t)), \dot{u}(t)\right\rangle-\langle\dot{f}(t), u(t)\rangle \\
& \geq-\left\|\nabla_{L^{2}} \mathcal{F}(t, u(t))\right\|_{L^{2}}\|\dot{u}(t)\|_{L^{2}}-\langle\dot{f}(t), u(t)\rangle \\
& \geq-\frac{1}{2}\|\dot{u}(t)\|_{L^{2}}^{2}-\frac{1}{2}\left\|\nabla_{L^{2}} \mathcal{F}(t, u(t))\right\|_{L^{2}}^{2}-\langle\dot{f}(t), u(t)\rangle .
\end{aligned}
$$

By (1.4) the above inequalities turn into equalities. In particular 


$$
\begin{aligned}
\left\langle\nabla_{L^{2}} \mathcal{F}(t, u(t)), \dot{u}(t)\right\rangle & =-\left\|\nabla_{L^{2}} \mathcal{F}(t, u(t))\right\|_{L^{2}}\|\dot{u}(t)\|_{L^{2}} \\
& =-\frac{1}{2}\|\dot{u}(t)\|_{L^{2}}^{2}-\frac{1}{2}\left\|\nabla_{L^{2}} \mathcal{F}(t, u(t))\right\|_{L^{2}}^{2} .
\end{aligned}
$$

By Cauchy inequality these identities imply $\dot{u}(t)=-\nabla_{L^{2}} \mathcal{F}(t, u(t))=A u(t)+$ $f(t)$.

Now, let us turn to our unilateral gradient flow, introducing the notion of weak solution. For sake of clarity, we start assuming again $f \in A C\left(0, T ; L^{2}\right)$. In this case, $u \in H^{1}\left(0, T ; L^{2}\right)$ is a unilateral gradient flow if the energy $t \mapsto$ $\mathcal{F}(t, u(t))$ is absolutely continuous and for a.e. $t \in(0, T)$ the following power estimate holds

$$
\dot{\mathcal{F}}(t, u(t)) \leq-\frac{1}{2}|\dot{u}(t)|_{L_{+}^{2}}^{2}-\frac{1}{2}|\partial \mathcal{F}|_{L_{+}^{2}}^{2}(t, u(t))-\langle\dot{f}(t), u(t)\rangle
$$

Here $|\dot{u}|_{L_{+}^{2}}$ and $|\partial \mathcal{F}|_{L_{+}^{2}}(t, u)$ denote respectively a "singular distance", which takes into account the unilateral constraint, and the unilateral slope respectively defined by

$$
|v|_{L_{+}^{2}}=\left\{\begin{array}{ll}
\|v\|_{L^{2}} & \text { if } v \geq 0, \\
+\infty & \text { otherwise, }
\end{array} \quad|\partial \mathcal{F}|_{L_{+}^{2}}(t, u)=\limsup _{|v-u|_{L_{+}^{2}} \rightarrow 0} \frac{[\mathcal{F}(t, v)-\mathcal{F}(t, u)]_{-}}{|v-u|_{L_{+}^{2}}} .\right.
$$

If $|\partial \mathcal{F}|_{L_{+}^{2}}(t, u)$ is finite then $A u+f(t)$ is a Radon measure with positive part in $L^{2}$ and $|\partial \mathcal{F}|_{L_{+}^{2}}(t, u)=\left\|[A u+f(t)]_{+}\right\|_{L^{2}}$ (see Corollary 3.8). Therefore, inequality (1.5) reads, in analogy with (1.4),

$$
\dot{\mathcal{F}}(t, u(t)) \leq-\frac{1}{2}\|\dot{u}(t)\|_{L^{2}}^{2}-\frac{1}{2}\left\|[A u(t)+f(t)]_{+}\right\|_{L_{+}^{2}}^{2}(t, u(t))-\langle\dot{f}(t), u(t)\rangle .
$$

Note that in the above inequality we cannot write the identity $A u(t)+f(t)=$ $-\nabla_{L^{2}} \mathcal{F}(t, u(t))$ since in general $A u(t) \notin L^{2}$ and thus the $L^{2}$-gradient is not well defined.

When $f \in L^{2}\left(0, T ; L^{2}\right)$ the power balance inequality (1.5) does not make sense since the time derivative of $f$ is not available. However, the same role is played by the power balance

$$
\dot{\mathcal{E}}(u(t)) \leq-\frac{1}{2}|\dot{u}(t)|_{L_{+}^{2}}^{2}-\frac{1}{2}|\partial \mathcal{F}|_{L_{+}^{2}}^{2}(t, u(t))+\langle f(t), \dot{u}(t)\rangle .
$$

where $\mathcal{E}(u)=\frac{1}{2} a(u, u)$. To be precise, in Definition 2.2 we will employ the time-integral formulation of (1.6) which is more convenient in the proofs, i.e.,

$$
\mathcal{E}\left(u\left(t^{*}\right)\right) \leq \mathcal{E}\left(u_{0}\right)-\frac{1}{2} \int_{0}^{t^{*}}|\dot{u}(t)|_{L_{+}^{2}}^{2}+|\partial \mathcal{F}|_{L_{+}^{2}}^{2}(t, u(t)) d t+\int_{0}^{t^{*}}\langle f(t), \dot{u}(t)\rangle d t
$$

for every $t^{*} \in(0, T)$.

As we will see, for any $f \in L^{2}\left(0, T ; L^{2}\right)$ and any initial datum $u_{0} \in H_{0}^{1}$ there exists a unique solution $u \in H^{1}\left(0, T ; L^{2}\right)$ satisfying (1.6) (see Theorem 2.3). For the solution $u$ the power estimate (1.6) actually holds as equality. Moreover, (see Theorem 2.5) $u$ solves also the parabolic equation

$$
\dot{u}(t)=[A u(t)+f(t)]_{+} \text {a.e. in }(0, T) .
$$

Noteworthy, the above parabolic equation is not equivalent to (1.6), in particular it may have many solutions (an explicit example is given in Sect. 5.2). In 
other terms, (1.6) provides a criterion to select a unique solution among those of the parabolic equation.

Now, let us describe in detail the structure and the content of the article. Sections and results are organized according to the time regularity of the datum $f$, which plays an important role (both in the analysis and in the applications). First of all we consider the most general case, i.e., $f \in L^{2}\left(0, T ; L^{2}\right)$, which occupies most of the paper. We prove existence and uniqueness of a solution in the sense of (1.6). Existence is obtained by time discretization, employing three different incremental problems of interest in the applications $[3,17,26]$. Let $t_{n, k}=k \tau_{n}$ be a uniform discretization of the interval $[0, T]$ with $\tau_{n}=T / n$. In the first scheme, given $u_{n, k}$ at time $t_{n, k}$, the configuration $u_{n, k+1}$ at time $t_{n, k+1}$ is simply given by

$$
u_{n, k+1} \in \operatorname{argmin}\left\{\mathcal{F}\left(t_{n, k+1}, u\right)+\frac{1}{2 \tau_{n}}\left|u-u_{n, k}\right|_{L_{+}^{2}}^{2}: u \in H_{0}^{1}\right\} .
$$

In the second scheme, we employ instead an a posteriori truncation, i.e., given $u_{n, k}$ we define $u_{n, k+1}$ by

$$
\left\{\begin{array}{l}
\tilde{u}_{n, k+1} \in \operatorname{argmin}\left\{\mathcal{F}\left(t_{n, k+1}, u\right)+\frac{1}{2 \tau_{n}}\left\|u-u_{n, k}\right\|_{L^{2}}^{2}: u \in H_{0}^{1}\right\} \\
u_{n, k+1}=\max \left\{\tilde{u}_{n, k+1}, u_{n, k}\right\} .
\end{array}\right.
$$

The fact that the first minimization is unconstrained makes this scheme very convenient in the numerical implementation [3,26], on the other hand the analysis is slightly more involved. Last, we consider a penalty method, i.e., given $u_{n, k}$ we get $u_{n, k+1}$ by solving,

$$
u_{n, k+1} \in \operatorname{argmin}\left\{\mathcal{F}\left(t_{n, k+1}, u\right)+\frac{1}{2 \tau_{n}}\left|u-u_{n, k}\right|_{L_{\alpha_{n}}^{2}}^{2}: u \in H_{0}^{1}\right\},
$$

where $\alpha_{n} \rightarrow+\infty$ and

$$
|v|_{L_{\alpha}^{2}}^{2}=\int_{\Omega} \psi_{\alpha}(v) d x \quad \text { and } \quad \psi_{\alpha}(v)= \begin{cases}v^{2} & \text { if } v \geq 0 \\ \alpha v^{2} & \text { if } v<0\end{cases}
$$

Each scheme defines a sequence of discrete solutions $u_{n}$ (depending on $\tau_{n}$ ) which enjoys suitable compactness properties and which converges (weakly and up to subsequences) to a solution of (1.6); a posteriori we actually prove that the whole sequence converges.

Note that for $f \in L^{2}\left(0, T ; L^{2}\right)$ the time regularity of solutions is rather low, since in general $u \in H^{1}\left(0, T ; L^{2}\right)$. As a consequence, uniqueness does not follow from classical tools, we use instead a contradiction argument [19] based on energy balance and convexity. For the same reason, the energy identity does not follow by the chain rule, which would require at least $u \in H_{l o c}^{1}\left(0, T ; H^{1}\right)$, rather, it is proved employing a measure theory argument, employed also in $[12,22]$.

In the second case we consider $f \in A C\left(0, T ; L^{2}\right)$. Existence and uniqueness is obviously contained in the previous one, however, from a theoretical point of view, it is interesting to know that in this case solutions are of class $H_{l o c}^{1}\left(0, T ; H_{0}^{1}\right) \cap W_{l o c}^{1, \infty}\left(0, T ; L^{2}\right)$; as a consequence, a better representation holds and few issues, due to the lack of time regularity, are avoided. 
Lastly, when $f$ is independent of time, besides recovering the classical result of [18], we prove a (rather surprising) property: the unique solution of (1.6) turns out to coincide with the unique solution of the unconstrained $L^{2}$ gradient flow for the functional $\tilde{\mathcal{F}}(u)=\mathcal{F}(u)+I_{+}\left(u-u_{0}\right)$, in other terms, the monotonicity constraint can be replaced by a fixed obstacle, given by the initial datum $u_{0}$; this property, however, does not hold when $f$ depends on time.

To complete our analysis, we prove a comparison principle and a (nonquantitative) continuous dependence property for solutions of (1.6); moreover, for the interested reader, we provide in the appendix further properties, representations and remarks on the unilateral slope.

\section{Setting and statement of the main results}

Let us consider an open, bounded domain $\Omega \subset \mathbb{R}^{d}$. Throughout the paper we employ the shorthand notation $L^{2}$ for $L^{2}(\Omega)$ and similarly for other functional spaces. We use the notation $\langle\cdot, \cdot\rangle$ for the scalar product in $L^{2}$, while $(\cdot, \cdot)$ denotes the duality between $H^{-1}$ and $H_{0}^{1}$. We consider a coercive, continuous and symmetric bi-linear form $a(\cdot, \cdot)$ in $H_{0}^{1} \times H_{0}^{1}$ given by

$$
a(u, v)=\int_{\Omega} \nabla u(x) \cdot B(x) \nabla v(x)+b(x) u(x) v(x) d x
$$

where $B=B^{T} \in L^{\infty}\left(\Omega, \mathbb{R}^{d \times d}\right)$ and $b \in L^{\infty}(\Omega)$; in order to guarantee coercivity we assume that $B$ is positive definite (i.e., there exists $C_{B}>0$ such that $\xi \cdot B(x) \xi \geq C_{B}|\xi|^{2}$, for every $\xi \in \mathbb{R}^{d}$ and a.e. $x \in \Omega$ ) and that $C_{\Omega}$ ess-inf $\{b\}+C_{B}>0$, where $C_{\Omega}$ is the Poincaré constant of $\Omega$. Let $A$ : $H_{0}^{1} \rightarrow H^{-1}$ be the associated operator, given by $(A u, v)=-a(u, v)$. Accordingly, we introduce the stored energy $\mathcal{E}: H_{0}^{1} \rightarrow \mathbb{R}$ given by

$$
\mathcal{E}(u)=\frac{1}{2} a(u, u)=\frac{1}{2} \int_{\Omega} \nabla u \cdot B \nabla u+b u^{2} d x .
$$

In (2.1) and in the sequel we drop, whenever possible, the dependence on $x \in \Omega$. Clearly $a^{1 / 2}(u, u)$ is the energy-norm which is equivalent to the standard norm in $H_{0}^{1}$.

Let $[0, T]$ be a time interval and let $f \in L^{2}\left(0, T ; L^{2}\right)$. Let us choose a representative of $f$ (defined for every $t \in[0, T]$ ) and consider the free energy $\mathcal{F}:[0, T] \times L^{2} \rightarrow \mathbb{R} \cup\{+\infty\}$ given by

$$
\mathcal{F}(t, u)= \begin{cases}\mathcal{E}(u)-\langle f(t), u\rangle & \text { for } u \in H_{0}^{1}, \\ +\infty & \text { otherwise }\end{cases}
$$

(in the sequel we will see that the evolution is actually independent of the choice of the representative). Clearly, for $u, v \in H_{0}^{1}$ and $t \in[0, T]$ the Fréchet differential of $\mathcal{F}(t, \cdot)$ reads

$$
d \mathcal{F}(t, u)[v]=a(u, v)-\langle f(t), v\rangle=-(A u+f(t), v) .
$$


In particular, if $A u \in L^{2}$

$$
-d \mathcal{F}(t, u)[z]=\langle A u+f(t), z\rangle=\left\langle-\nabla_{L^{2}} \mathcal{F}(t, u), z\right\rangle .
$$

Here, following the theory of curves of maximal slope [5] we provide first a notion of unilateral slope. To this end, for $w \in L^{2}$ let us introduce the following notation

$$
|w|_{L_{+}^{2}}= \begin{cases}\|w\|_{L^{2}} & \text { if } w \geq 0 \\ +\infty & \text { otherwise. }\end{cases}
$$

Accordingly, we will say that $v \rightarrow u$ in $L_{+}^{2}$ when $|v-u|_{L_{+}^{2}} \rightarrow 0$, i.e. when $v \geq u$ and $v \rightarrow u$ in $L^{2}$. Finally, let us introduce the unilateral $L^{2}$-slope, defined as follows.

Definition 2.1. (Unilateral slope) For $u \in H_{0}^{1}$ let

$$
|\partial \mathcal{F}|_{L_{+}^{2}}(t, u)=\limsup _{v \rightarrow u} \frac{[\mathcal{F}(t, v)-\mathcal{F}(t, u)]_{-}}{|v-u|_{L_{+}^{2}}},
$$

where $[\cdot]_{-}$denotes negative part and $v \rightarrow u$ in $L_{+}^{2}$. For convenience, set $|\partial \mathcal{F}|_{L_{+}^{2}}(t, u)=+\infty$ if $u \notin H_{0}^{1}$.

In Sect. 3 we will see that $|\partial \mathcal{F}|_{L_{+}^{2}}(t, u)=\sup \left\{-d \mathcal{F}(t, u)[z]: z \in H_{0}^{1},|z|_{L_{+}^{2}} \leq 1\right\}=\left\|[A u+f(t)]_{+}\right\|_{L^{2}}$, whenever $A u+f(t)$ is a locally finite Radon measure with positive part in $L^{2}$. For equivalent ways of writing the slope, in terms of a "singular metric" and in terms of a "unilateral subdifferential", see Appendix A and B.

\subsection{The case $f \in L^{2}\left(0, T ; L^{2}\right)$}

Definition 2.2. An evolution $u \in L^{\infty}\left(0, T ; H_{0}^{1}\right) \cap H^{1}\left(0, T ; L^{2}\right)$ is a unilateral gradient flow for the energy $\mathcal{F}$ if for every $0 \leq t^{*} \leq T$ it holds

$$
\begin{aligned}
\mathcal{E}\left(u\left(t^{*}\right)\right) \leq & \mathcal{E}(u(0))-\frac{1}{2} \int_{0}^{t^{*}}|\dot{u}(t)|_{L_{+}^{2}}^{2}+|\partial \mathcal{F}|_{L_{+}^{2}}^{2}(t, u(t)) d t \\
& +\int_{0}^{t^{*}}\langle f(t), \dot{u}(t)\rangle d t .
\end{aligned}
$$

Upon identifying $u \in H^{1}\left(0, T ; L^{2}\right)$ with its $A C$ representative, for every $0 \leq t_{1} \leq t_{2} \leq T$ we can write

$$
u\left(t_{2}\right)=u\left(t_{1}\right)+\int_{t_{1}}^{t_{2}} \dot{u}(t) d t
$$

where the identity holds in $L^{2}$ (and thus a.e. in $\Omega$ ). If $\dot{u}(t) \geq 0$ (a.e. in $\Omega$ ) for a.e. $t \in(0, T)$ then $u\left(t_{2}\right) \geq u\left(t_{1}\right)$ (a.e. in $\Omega$ ) for every $0 \leq t_{1} \leq t_{2} \leq T$, and thus we will say that $u$ is monotone in $[0, T]$. The fact that the evolution $u$, appearing in Definition 2.2 , is monotone in $[0, T]$ is implicitly written in $(2.5)$; indeed for $t^{*}=T(2.5)$ implies that

$$
\frac{1}{2} \int_{0}^{T}|\dot{u}(t)|_{L_{+}^{2}}^{2} d t \leq \mathcal{E}(u(0))+\|f\|_{L^{2}\left(0, T ; L^{2}\right)}\|\dot{u}\|_{L^{2}\left(0, T ; L^{2}\right)}<+\infty,
$$


then $|\dot{u}(t)|_{L_{+}^{2}}<+\infty$ for a.e. $t \in(0, T)$, i.e., $\dot{u}(t) \geq 0$ for a.e. $t \in(0, T)$.

Note that (2.5) is independent of the choice of the representative of the datum $f$.

The next theorem contains the main result: existence, uniqueness, and power identity.

Theorem 2.3. Given $u_{0} \in H_{0}^{1}$ there exists a unique unilateral gradient flow $u$ for $\mathcal{F}$ with $u(0)=u_{0}$. Moreover, the energy $t \mapsto \mathcal{E}(u(t))$ is absolutely continuous in $[0, T]$ and for a.e. $t \in(0, T)$ it holds

$$
\begin{aligned}
\dot{\mathcal{E}}(u(t)) & =-|\dot{u}(t)|_{L_{+}^{2}}|\partial \mathcal{F}|_{L_{+}^{2}}(t, u(t))+\langle f(t), \dot{u}(t)\rangle \\
& =-\frac{1}{2}|\dot{u}(t)|_{L_{+}^{2}}^{2}-\frac{1}{2}|\partial \mathcal{F}|_{L_{+}^{2}}^{2}(t, u(t))+\langle f(t), \dot{u}(t)\rangle .
\end{aligned}
$$

Note that the above power identity implies that $|\dot{u}(t)|_{L_{+}^{2}}=|\partial \mathcal{F}|_{L_{+}^{2}}(t, u(t))$.

The proof of the above theorem is split in different sections: existence is proved by means of three different time-discrete schemes, contained in Sects. 4.1, 4.2, and 4.3, while energy identity and uniqueness are proved in Sect. 4.4 .

In order to better understand and justify the above definition of solution we state here the following results, dealing respectively with strong and weak solutions of the parabolic problem $\dot{u}(t)=[A u(t)+f(t)]_{+}$.

Theorem 2.4. Let $u_{0} \in H_{0}^{1}$. Let $u \in H^{1}\left(0, T ; L^{2}\right)$ with $u(0)=u_{0}$ and $A u(t)+$ $f(t) \in L^{2}$ for a.e. $t \in(0, T)$. Then $u$ is a unilateral gradient flow in the sense of Definition 2.2 if and only if it solves the parabolic problem

$$
\left\{\begin{array}{l}
\dot{u}(t)=[A u(t)+f(t)]_{+} \quad \text { in } L^{2} \text { for a.e. } t \in(0, T), \\
u(0)=u_{0},
\end{array}\right.
$$

Theorem 2.5. Let $u$ be the unilateral gradient flow provided by Theorem 2.3, then $u$ solves the parabolic partial differential equation

$$
\left\{\begin{array}{l}
\dot{u}(t)=[A u(t)+f(t)]_{+} \quad \text { in } L^{2} \text { for a.e. } t \in(0, T), \\
u(0)=u_{0},
\end{array}\right.
$$

where $A u(t)+f(t)$ is a (locally finite) Radon measure and $[A u(t)+f(t)]_{+} \in L^{2}$ is its positive part.

Remark 2.6. As far as Theorem 2.4, note that in general $A u(t)+f(t) \in H^{-1} \backslash$ $L^{2}$ for a.e. $t \in(0, T)$; an explicit example is given in Sect. 5.1. In particular, if $\Psi: L^{2} \rightarrow[0,+\infty]$ is given by $\Psi(v)=\frac{1}{2}\|v\|_{L^{2}}^{2}+I_{+}(v)$ (where $I_{+}$is the indicator function of the set $\{v \geq 0\})$ it is not always possible to re-write (2.7) in the form

$$
\left\{\begin{array}{l}
\partial \Psi(\dot{u}(t)) \ni A u(t)+f(t) \quad \text { in } L^{2} \text { for a.e. } t \in(0, T), \\
u(0)=u_{0},
\end{array}\right.
$$

since $\partial \Psi \subset L^{2}$ and thus $A u(t)+f(t)$ should be in $L^{2}$. The latter equation, in the form

$$
\dot{u}(t)+\partial I_{+}(\dot{u}(t))-A u(t)-f(t) \ni 0,
$$


is adopted e.g. in $[1,2]$ under stronger regularity on the data (e.g. if $u_{0} \in H^{2}$ and $f$ is suitably controlled) which ensure $A u(t)+f(t)$ in $L^{2}$ for a.e. $t \in(0, T)$, see e.g. [2, Theorem 2.6].

Remark 2.7. In Sect.5.1 we will see that solutions of (2.8) are not unique. Lack of uniqueness is essentially due to the unilateral constraint, since the solution of the unconstrained gradient flow for $\mathcal{F}$ would be unique (see e.g. $[10])$.

Remark 2.8. Due to the lack of time regularity of the datum $f$, in general the energy $t \mapsto \mathcal{F}(t, u(t))$ is not absolutely continuous, for this reason in (2.6) we employ the derivative of the energy $t \mapsto \mathcal{E}(u(t))$. The absolute continuity of the energy $t \mapsto \mathcal{F}(t, u(t))$ is recovered under more restrictive assumptions of the datum $f$, for instance when $f \in A C\left(0, T ; L^{2}\right)$, see Proposition 2.12. Finally, note that (2.6) can be equivalently written in integral form as: for every $0 \leq t^{*} \leq T$ it holds

$$
\begin{aligned}
\mathcal{E}\left(u\left(t^{*}\right)\right)= & \mathcal{E}(u(0))-\frac{1}{2} \int_{0}^{t^{*}}|\dot{u}(t)|_{L_{+}^{2}}^{2}+|\partial \mathcal{F}|_{L_{+}^{2}}^{2}(t, u(t)) d t \\
& +\int_{0}^{t^{*}}\langle f(t), \dot{u}(t)\rangle d t
\end{aligned}
$$

therefore the energy inequality (2.5) turns into an equality.

Remark 2.9. From the physical point of view solutions in the sense of Definition 2.2 could be equivalently characterized as a solutions to the parabolic problem (2.8) satisfying the energy identity

$$
\mathcal{E}\left(u\left(t^{*}\right)\right)=\mathcal{E}\left(u_{0}\right)-\int_{0}^{t^{*}} \mathcal{D}(\dot{u}(t)) d t+\int_{0}^{t^{*}} \mathcal{P}_{\text {ext }}(t, \dot{u}(t)) d t,
$$

where

$$
\mathcal{D}(\dot{u}(t))=|\dot{u}(t)|_{L_{+}^{2}}^{2} \quad \text { and } \quad \mathcal{P}_{\text {ext }}(t, \dot{u}(t))=\langle f(t), \dot{u}(t)\rangle
$$

denote respectively the dissipation and the power of external forces.

Unilateral gradient flows, in the sense of Definition 2.2, enjoy comparison principle and continuous dependence; on the contrary, by lack of uniqueness, solutions of (2.8) do not satisfy them.

Proposition 2.10. Let $u$ denote the unilateral gradient flow with initial condition $u_{0} \in H_{0}^{1}$ and data $f \in L^{2}\left(0, T ; L^{2}\right)$. Let $v$ denote the unilateral gradient flow with initial condition $v_{0} \in H_{0}^{1}$ and data $g \in L^{2}\left(0, T ; L^{2}\right)$. If $u_{0} \leq v_{0}$ and $f \leq g$ then $u \leq v$ in $[0, T]$.

Proposition 2.11. Let $f^{m} \rightarrow f$ in $L^{2}\left(0, T ; L^{2}\right)$ and $u_{0}^{m} \rightarrow u_{0}$ in $H_{0}^{1}$; let $u^{m}$ and $u$ be the corresponding unilateral gradient flows. Then $u^{m} \rightarrow u$ in $H^{1}\left(0, T ; L^{2}\right)$ and $u^{m}(t) \rightarrow u(t)$ in $H_{0}^{1}$ for every $t \in[0, T]$. 


\subsection{The case $f \in A C\left(0, T ; L^{2}\right)$}

If $f \in A C\left(0, T ; L^{2}\right)$ the results of Theorems 2.3 and 2.5 can be improved.

Proposition 2.12. Let $f \in A C\left(0, T ; L^{2}\right)$ and let $u$ be a unilateral gradient flow in the sense of Definition 2.2, then the energy $t \mapsto \mathcal{F}(t, u(t))$ is absolutely continuous in $[0, T]$ and

$$
\dot{\mathcal{F}}(t, u(t)) \leq-\frac{1}{2}|\dot{u}(t)|_{L_{+}^{2}}^{2}-\frac{1}{2}|\partial \mathcal{F}|_{L_{+}^{2}}^{2}(t, u(t))-\langle\dot{f}(t), u(t)\rangle .
$$

Moreover, following [18], the unique solution u of Theorem 2.3 is also the unique solution in $H^{1}\left(0, T ; L^{2}\right) \cap H_{l o c}^{1}\left(0, T ; H_{0}^{1}\right)$ of

$$
\left\{\begin{array}{l}
\partial \Phi(\dot{u}(t)) \ni A u(t)+f(t) \quad \text { in } H^{-1} \text { for a.e. } t \in(0, T), \\
u(0)=u_{0},
\end{array}\right.
$$

where $\Phi: H_{0}^{1} \rightarrow[0,+\infty]$ is defined by $\Phi(u)=\frac{1}{2}\|u\|_{L^{2}}^{2}+I_{+}(u)$ and $\partial \Phi(u) \subset$ $H^{-1}$ denotes its subdifferential. Finally, the solution $u$ belongs also to the space $W_{l o c}^{1, \infty}\left(0, T ; L^{2}\right)$.

Inequality (2.12) provides, in the non-autonomous case, a notion of curve of maximal (unilateral) slope corresponding to [5, Definition 1.3.2]. Moreover, as a consequence of Proposition 2.12, for every $0 \leq t^{*} \leq T$ the energy identity reads

$$
\begin{aligned}
\mathcal{F}\left(t^{*}, u\left(t^{*}\right)\right)= & \mathcal{F}(0, u(0))-\frac{1}{2} \int_{0}^{t^{*}}|\dot{u}(t)|_{L_{+}^{2}}^{2}+|\partial \mathcal{F}|_{L_{+}^{2}}^{2}(t, u(t)) d t \\
& -\int_{0}^{t^{*}}\langle\dot{f}(t), u(t)\rangle d t .
\end{aligned}
$$

\subsection{The case $f$ independent of time}

The existence and uniqueness of solutions when $f$ is independent of time has been already treated in the literature, see e.g. [18]; however, in this case we show that the constraint on $\dot{u}$ can be replaced by a fixed obstacle, as a consequence we provide a further and simple characterization (2.15) of solutions, in the spirit of the recent [1, Remark 5.3] but with a different proof.

Proposition 2.13. Let $f \in L^{2}$ and $\mathcal{F}(u)=\frac{1}{2} a(u, u)-\langle f, u\rangle$ (both independent of time). Given $u_{0} \in H_{0}^{1}$, let $u \in H^{1}\left(0, T ; L^{2}\right) \cap L^{\infty}\left(0, T ; H_{0}^{1}\right)$ be the unilateral gradient flow for $\mathcal{F}$ with initial datum $u_{0}$.

Then, $u$ turns out to be the (unconstrained) $L^{2}$-gradient flow for the functional $\tilde{\mathcal{F}}(u)=\mathcal{F}(u)+I_{+}\left(u-u_{0}\right)$. Moreover, $u$ is also the unique solution of the following parabolic obstacle problem:

$$
\begin{cases}\dot{u}(t)-A u(t)-f \geq 0 & \text { in } H^{-1} \text { for a.e. } t \in(0, T) \\ \left(u(t)-u_{0}, \dot{u}(t)-A u(t)-f\right)=0 & \text { for a.e. } t \in(0, T) \\ u(0)=u_{0}, \quad u(t) \geq u_{0} & \text { for a.e. } t \in(0, T) .\end{cases}
$$

As we will see in Sect. 7 the above characterization does not hold when the datum $f$ depends on time. 


\subsection{Generalizations and applications}

To conclude this section, let us mention that several of the above results can be extended or partially extended to more general functionals, with few modifications in the definitions and in proofs. The choice of quadratic functionals is motivated by sake of simplicity and by the fact that quadratic, or separately quadratic, energies are mostly used in applications.

As far as generalizations, let us mention the following. Let $p \in(1,+\infty)$ such that $W^{1, p} \subset L^{2}$ (by Sobolev embedding), let $f \in L^{2}\left(0, T ; L^{2}\right)$ and consider $w: \mathbb{R} \rightarrow[0,+\infty)$ to be $\lambda$-convex, i.e. (see [5, Definition 2.4.1]),

$$
w\left(s z_{1}+(1-s) z_{0}\right) \leq s w\left(z_{1}\right)+(1-s) w\left(z_{0}\right)-\frac{1}{2} \lambda s(1-s)\left(z_{1}-z_{0}\right)^{2},
$$

for some $\lambda<0$ and for every $z_{0}, z_{1} \in \mathbb{R}$ and $s \in(0,1)$. The double-well potential $w(z)=z^{2}(z-1)^{2}$, appearing in the Allen-Cahn equation, is a prototype $\lambda$-convex functions for $\lambda \leq \min _{z} w^{\prime \prime}(z)$. Under these assumptions, we can define the stored energy $\mathcal{E}$ and the free energy $\mathcal{F}$ respectively given by

$$
\mathcal{E}(u)=\int_{\Omega}|\nabla u|^{p}+w(u) d x, \quad \mathcal{F}(t, u)=\mathcal{E}(u)-\langle f(t), u\rangle .
$$

In this case, adapting the arguments of the following sections, it is not difficult to see that for $u_{0} \in W_{0}^{1, p}$ there exists an evolution $u \in H^{1}\left(0, T ; L^{2}\right) \cap$ $L^{\infty}\left(0, T ; W^{1, p}\right)$ which satisfies the energy identity $(2.10)$. However, in this weak setting, uniqueness is still open since the arguments of Sect. 4.4 do not apply.

As far as applications, let us mention the phase-field approach to damage and fracture, based on the "Ambrosio-Tortorelli" energy [6]

$$
\mathcal{F}(t, u, v)=\frac{1}{2} \int_{\Omega}|\nabla v|^{2}+v^{2} d x+\int_{\Omega}\left((v-1)^{2}+\eta\right)|\nabla u+\nabla g(t)|^{2} d x .
$$

In this context the unilateral gradient flow takes the form $[3,8,21]$

$$
\dot{v}(t)=[\Delta v(t)-v(t)-F(t, v(t))]_{+}
$$

where $F(t, v)=(v-1)|\nabla u(t, v)+\nabla g(t)|^{2}$ and

$$
u(t, v) \in \operatorname{argmin}\left\{\int_{\Omega}\left((v-1)^{2}+\eta\right)|\nabla u+\nabla g(t)|^{2} d x: u \in H_{0}^{1}\right\} .
$$

Here the monotonicity constraint models irreversibility, with $v=0$ corresponding to the sound material and $v=1$ to the fully damaged or fully cracked material. We remark that, beside the non-linearity, the regularity of the forcing term $t \mapsto F(t, v(t))$ is usually quite low.

\section{Energy and unilateral slope}

To the purpose of this section, fix $t \in[0, T]$ and let $f(t) \in L^{2}$. If $u \in H_{0}^{1}$ the differential of $\mathcal{F}(t, \cdot)$ restricted to $H_{0}^{1}$ is

$$
d \mathcal{F}(t, u)[z]=a(u, z)-\langle f(t), z\rangle=-(A u+f(t), z) \quad \text { for } z \in H_{0}^{1} .
$$


Lemma 3.1. If $u \in H_{0}^{1}$ then

$$
\begin{aligned}
|\partial \mathcal{F}|_{L_{+}^{2}}(t, u) & =\sup \left\{-d \mathcal{F}(t, u)[z]: z \in H_{0}^{1},|z|_{L_{+}^{2}} \leq 1\right\} \\
& =\sup \left\{-d \mathcal{F}(t, u)[z]: z \in H_{0}^{1}, z \geq 0,\|z\|_{L^{2}} \leq 1\right\} .
\end{aligned}
$$

Proof. Denote $S=\sup \left\{-d \mathcal{F}(t, u)[z]: z \in H_{0}^{1},|z|_{L_{+}^{2}} \leq 1\right\}$. Since $z=0$ is an admissible variation, it is clear that $S \in[0,+\infty]$.

Given $z \neq 0$ as in (3.2) let $v_{s}=u+s z$ for $s \geq 0$. Then, being $[r]_{-} \geq-r$ for $r \in \mathbb{R}$

$$
\begin{aligned}
|\partial \mathcal{F}|_{L_{+}^{2}}(t, u) & \geq \limsup _{s \rightarrow 0} \frac{\left[\mathcal{F}\left(t, v_{s}\right)-\mathcal{F}(t, u)\right]_{-}}{\left\|v_{s}-u\right\|_{L^{2}}} \\
& \geq \limsup _{s \rightarrow 0} \frac{\mathcal{F}(t, u)-\mathcal{F}(t, u+s z)}{s}=-d \mathcal{F}(t, u)[z] .
\end{aligned}
$$

Taking the supremum on the right hand side we get $|\partial \mathcal{F}|_{L_{+}^{2}}(t, u) \geq S$.

Let us prove that $|\partial \mathcal{F}|_{L_{+}^{2}}(t, u) \leq S$. If $|\partial \mathcal{F}|_{L_{+}^{2}}(t, u)=0$ there is nothing to prove. Otherwise, let $v_{n} \rightarrow u$ with $v_{n} \geq u$ such that $0<|\partial \mathcal{F}|_{L_{+}^{2}}(t, u)=$ $\lim _{n \rightarrow+\infty}\left[\mathcal{F}\left(t, v_{n}\right)-\mathcal{F}(t, u)\right]_{-} /\left\|v_{n}-u\right\|_{L^{2}}$. Hence, $\mathcal{F}\left(t, v_{n}\right)-\mathcal{F}(t, u)<0$ for $n \gg 1$. By convexity, $\mathcal{F}\left(t, v_{n}\right) \geq \mathcal{F}(t, u)+d \mathcal{F}(t, u)\left[v_{n}-u\right]$ and then, for $n \gg 1$,

$$
\begin{aligned}
\frac{\left[\mathcal{F}\left(t, v_{n}\right)-\mathcal{F}(t, u)\right]_{-}}{\left\|v_{n}-u\right\|_{L^{2}}} & =\frac{\mathcal{F}(t, u)-\mathcal{F}\left(t, v_{n}\right)}{\left\|v_{n}-u\right\|_{L^{2}}} \\
& \leq \frac{-d \mathcal{F}(t, u)\left[v_{n}-u\right]}{\left\|v_{n}-u\right\|_{L^{2}}}=-d \mathcal{F}(t, u)\left[\xi_{n}\right] \leq S
\end{aligned}
$$

where $\xi_{n}=\left(v_{n}-u\right) /\left\|v_{n}-u\right\|_{L^{2}}$ belongs to $H_{0}^{1}, \xi_{n} \geq 0$ and $\left\|\xi_{n}\right\|_{L^{2}} \leq 1$.

As $u \mapsto-d \mathcal{F}(t, u)[z]$ is linear, taking the supremum in (3.2) yields also the following Corollary.

Corollary 3.2. The map $u \mapsto|\partial \mathcal{F}|_{L_{+}^{2}}(t, u)$ is convex in $H_{0}^{1}$.

Remark 3.3. If $t \mapsto u(t)$ is measurable from $[0, T]$ to $H_{0}^{1}$ then the map $t \mapsto$ $|\partial \mathcal{F}|_{L_{+}^{2}}(t, u(t))$ is measurable. Indeed, given $z \in H_{0}^{1}$ with $z \geq 0$ and $\|z\|_{L^{2}} \leq 1$ the map

$$
t \mapsto-d \mathcal{F}(t, u(t))[z]=-\int_{\Omega} \nabla u(t) \cdot B \nabla z+b u(t) z d x+\int_{\Omega} f(t) z d x
$$

is measurable. Taking a dense countable subset $\left\{z_{n}\right\}$ of $\left\{z \in H_{0}^{1}: z \geq 0\right.$ and $\left.\|z\|_{L^{2}} \leq 1\right\}$ yields

$$
|\partial \mathcal{F}|_{L_{+}^{2}}(t, u(t))=\sup _{n \in \mathbb{N}}\left\{-d \mathcal{F}(t, u(t))\left[z_{n}\right]\right\}
$$

where the supremum is taken pointwise in $[0, T]$. Measurability follows.

Corollary 3.4. Let $f_{n}(t) \rightarrow f(t)$ in $L^{2}$ and consider the energies

$$
\mathcal{F}_{n}(t, u)=\frac{1}{2} a(u, u)-\left\langle f_{n}(t), u\right\rangle, \quad \mathcal{F}(t, u)=\frac{1}{2} a(u, u)-\langle f(t), u\rangle .
$$

If $u_{n} \rightarrow u$ in $H_{0}^{1}$ then

$$
\mathcal{F}(t, u) \leq \liminf _{n \rightarrow+\infty} \mathcal{F}_{n}\left(t, u_{n}\right), \quad|\partial \mathcal{F}|_{L_{+}^{2}}(t, u) \leq \liminf _{n \rightarrow+\infty}\left|\partial \mathcal{F}_{n}\right|_{L_{+}^{2}}\left(t, u_{n}\right) .
$$


Proof. The weak lower semicontinuity of the energy is obvious. Let $z \in H_{0}^{1}$ with $z \geq 0$ and $\|z\|_{L^{2}} \leq 1$. If $f_{n}(t) \rightarrow f(t)$ and $u_{n} \rightarrow u$ in $H_{0}^{1}$ then we get

$$
\begin{aligned}
d \mathcal{F}(t, u)[z] & =a(u, z)-\langle f(t), z\rangle=\lim _{n \rightarrow+\infty} a\left(u_{n}, z\right)-\left\langle f_{n}(t), z\right\rangle \\
& =\lim _{n \rightarrow+\infty} d \mathcal{F}_{n}\left(t, u_{n}\right)[z] .
\end{aligned}
$$

By Lemma 3.1 we deduce that

$$
-d \mathcal{F}(t, u)[z]=\liminf _{n \rightarrow+\infty}-d \mathcal{F}_{n}\left(t, u_{n}\right)[z] \leq \liminf _{n \rightarrow+\infty}\left|\partial \mathcal{F}_{n}\right|_{L_{+}^{2}}\left(t, u_{n}\right),
$$

from which (3.3) follows by taking the supremum with respect to $z$.

Remark 3.5. The energy $\mathcal{F}(t, \cdot)$ is T-monotone, i.e. $\left(\xi_{u}-\xi_{v},[u-v]_{+}\right) \geq 0$ for $\xi_{u} \in \partial \mathcal{F}(t, u)$ and $\xi_{v} \in \partial \mathcal{F}(t, v)$, where $\partial \mathcal{F}(t, \cdot) \subset H^{-1}$ denotes the subdifferential in $H_{0}^{1}$. For the details, see [25, Remark 2.4].

To conclude, we provide in Corollary 3.8 an $L^{2}$ "representation" of the slope, which is fundamental to connect the unilateral gradient flow and the parabolic problem (2.8); its proof is a direct consequence of the next abstract lemmas on Radon measures.

Lemma 3.6. Let $\zeta \in H^{-1}$ with

$$
\sup \left\{(\zeta, z): z \in H_{0}^{1}, z \geq 0,\|z\|_{L^{2}} \leq 1\right\}<+\infty,
$$

then $\zeta$ is a (locally finite) Radon measure whose positive part $\zeta_{+}$belongs to $L^{2}$. Moreover

$$
\sup \left\{(\zeta, z): z \in H_{0}^{1}, z \geq 0,\|z\|_{L^{2}} \leq 1\right\}=\left\|\zeta_{+}\right\|_{L^{2}} .
$$

Proof. For the first part see [22, Lemma A.2] or [11]. We give a short proof of the second part. By density we can write

$$
\begin{aligned}
\sup & \left\{(\zeta, z): z \in H_{0}^{1}, z \geq 0,\|z\|_{L^{2}} \leq 1\right\} \\
= & \sup \left\{(\zeta, z): z \in C_{c}^{\infty}, z \geq 0,\|z\|_{L^{2}} \leq 1\right\} \\
= & \sup \left\{\int_{\Omega} z d \zeta: z \in C_{c}, z \geq 0,\|z\|_{L^{2}} \leq 1\right\} \\
= & \sup \left\{\int_{\Omega} z d \zeta_{+}: z \in C_{c}, z \geq 0,\|z\|_{L^{2}} \leq 1\right\} \\
= & \sup \left\{\int_{\Omega} z \zeta_{+} d x: z \in L^{2}, z \geq 0,\|z\|_{L^{2}} \leq 1\right\}=\left\|\zeta_{+}\right\|_{L^{2}},
\end{aligned}
$$

which concludes the proof.

Lemma 3.7. Let $\zeta$ be as in the previous lemma and $z \in L^{2}$ with $z \geq 0$ and $z \geq \zeta$ in $H^{-1}$. Then $z \geq \zeta_{+}$in $L^{2}$.

Proof. Since $(z-\zeta)$ is a positive measure, it follows that $\zeta$ is a measure as well. Write $\zeta=\zeta_{+}-\zeta_{-}$, where $\zeta_{ \pm}$are supported on the disjoint sets $\Omega_{ \pm}$. In particular we have $z \geq \zeta_{+}$as measures and functions in $\Omega_{+}$. As $z \geq 0$ it follows that $z \geq \zeta_{+}$also in $\Omega_{-}$. 
Invoking Lemma 3.6 together with Lemma 3.1 and (3.1) we get this Corollary.

Corollary 3.8. The following conditions are equivalent:

(i) $|\partial \mathcal{F}|_{L_{+}^{2}}(t, u)<+\infty$,

(ii) $-d \mathcal{F}(t, u)=A u+f(t)$ is a (locally finite) Radon measure such that $[A u+f(t)]_{+} \in L^{2}$.

Moreover $|\partial \mathcal{F}|_{L_{+}^{2}}(t, u)=\left\|[A u+f(t)]_{+}\right\|_{L^{2}}$.

\section{Solutions for $f \in L^{2}\left(0, T ; L^{2}\right)$}

In the following subsections we prove existence of unilateral gradient flows, in the sense of Definition 2.2, by means of three discrete schemes, which take into account the monotonicity constraint in different ways. We remark that all these ways of introducing monotonicity are currently employed in applications to phase-field fracture, with suitable adaptations. We provide complete proofs, however, those parts which are quite similar are not repeated.

\subsection{Constrained incremental problem}

Let $\tau_{n}=T / n$ and $t_{n, k}=k \tau_{n}$ for $k=0, \ldots, n$. First of all, for every index $k=0, \ldots, n-1$ define

$$
f_{n, k+1}=f_{t_{n, k}}^{t_{n, k+1}} f(t) d t .
$$

Let $f_{n} \in L^{2}\left(0, T ; L^{2}\right)$ given by $f_{n}(t)=f_{n, k+1}$ for every $t \in\left(t_{n, k}, t_{n, k+1}\right]$. Note that $f_{n} \rightarrow f$ in $L^{2}\left(0, T ; L^{2}\right)$ and that $f_{n}(t) \rightarrow f(t)$ in $L^{2}$ for a.e. $t \in(0, T)$. Denote by $\mathcal{F}_{n}$ the corresponding energy, i.e. $\mathcal{F}_{n}(t, u)=\frac{1}{2} a(u, u)-\left\langle f_{n}(t), u\right\rangle$. Note that $\mathcal{F}_{n}(t, \cdot)=\mathcal{F}_{n}\left(t_{n, k+1}, \cdot\right)$ for every $t \in\left(t_{n, k}, t_{n, k+1}\right]$.

Define $u_{n, 0}=u_{0}$ at time $t_{n, 0}$, and then, given $u_{n, k}$ at time $t_{n, k}$, let the configuration at time $t_{n, k+1}$ be given by

$$
u_{n, k+1} \in \operatorname{argmin}\left\{\mathcal{F}_{n}\left(t_{n, k+1}, u\right)+\frac{1}{2 \tau_{n}}\left|u-u_{n, k}\right|_{L_{+}^{2}}^{2}: u \in H_{0}^{1}\right\} .
$$

Note that a unique minimizer exists by standard arguments and that $u_{n, k+1} \geq$ $u_{n, k}$.

Define $u_{n}:[0, T] \rightarrow L^{2}$ and $u_{n}^{\sharp}:[0, T] \rightarrow L^{2}$ respectively as the piecewise affine interpolation and the piecewise constant backward (left-continuous) interpolation of the values $u_{n, k}$ in the points $t_{n, k}$. In this section we will prove the following proposition. We write for simplicity $u_{n, k+1}=u_{n}\left(t_{n, k+1}\right)$ and $\dot{u}_{n, k+1}=\left(u_{n, k+1}-u_{n, k}\right) / \tau_{n}$ instead of $\dot{u}_{n}(t)$ for $t \in\left(t_{n, k}, t_{n, k+1}\right)$.

Proposition 4.1. Let $u_{n}$ be defined as above. There exists a subsequence (not relabeled) and $u \in H^{1}\left(0, T ; L^{2}\right)$ such that $u_{n} \rightarrow u$ in $H^{1}\left(0, T ; L^{2}\right)$ where $u$ is a unilateral gradient flow in the sense of Definition 2.2 .

Remark 4.2. After Sect. 4.4 we will see that actually the whole sequence $u_{n}$ converges weakly to $u$ in $H^{1}\left(0, T ; L^{2}\right)$ and that both $u_{n}^{\sharp} \rightarrow u$ and $u_{n} \rightarrow u$ (strongly) in $H_{0}^{1}$ pointwise in $[0, T]$. 
Lemma 4.3. For every $t \in\left(t_{n, k}, t_{n, k+1}\right)$ it holds

$$
\left|\dot{u}_{n}(t)\right|_{L_{+}^{2}}^{2}=\left\|\dot{u}_{n}(t)\right\|_{L^{2}}^{2}=-d \mathcal{F}_{n}\left(t_{n, k+1}, u_{n, k+1}\right)\left[\dot{u}_{n}(t)\right]=\left|\partial \mathcal{F}_{n}\right|_{L_{+}^{2}}^{2}\left(t, u_{n}^{\sharp}(t)\right) .
$$

Proof. By definition $u_{n, k+1}$ is the solution (in $H_{0}^{1}$ ) of the variational problem

$$
d \mathcal{F}_{n}\left(t_{n, k+1}, u_{n, k+1}\right)\left[v-u_{n, k+1}\right]+\left\langle\dot{u}_{n, k+1}, v-u_{n, k+1}\right\rangle \geq 0
$$

for every $v \in H_{0}^{1}$ with $v \geq u_{n, k}$. Choosing $v=2 u_{n, k+1}-u_{n, k}$ and $v=u_{n, k}$ yields respectively $\left(v-u_{n, k+1}\right)=\tau_{n} \dot{u}_{n, k+1}$ and $\left(v-u_{n, k+1}\right)=-\tau_{n} \dot{u}_{n, k+1}$; hence

$$
d \mathcal{F}_{n}\left(t_{n, k+1}, u_{n, k+1}\right)\left[\tau_{n} \dot{u}_{n, k+1}\right]+\tau_{n}\left\|\dot{u}_{n, k+1}\right\|_{L^{2}}^{2}=0
$$

which gives the second equality in (4.2). Moreover, the variational inequality implies that

$$
-d \mathcal{F}_{n}\left(t_{n, k+1}, u_{n, k+1}\right)[z] \leq\left\langle\dot{u}_{n, k+1}, z\right\rangle \text { for every } z \in H_{0}^{1} \text { with } z \geq 0 .
$$

Then, by Lemma 3.1

$$
\begin{aligned}
& \left|\partial \mathcal{F}_{n}\right|_{L_{+}^{2}}\left(t_{n, k+1}, u_{n, k+1}\right) \\
& \quad=\sup \left\{-d \mathcal{F}_{n}\left(t_{n, k+1}, u_{n, k+1}\right)[z]: z \in H_{0}^{1}, z \geq 0,\|z\|_{L^{2}} \leq 1\right\} \\
& \quad \leq \sup \left\{\left\langle\dot{u}_{n, k+1}, z\right\rangle_{L^{2}}: z \in H_{0}^{1}, z \geq 0,\|z\|_{L^{2}} \leq 1\right\}=\left\|\dot{u}_{n, k+1}\right\|_{L^{2}} .
\end{aligned}
$$

Assume that $\dot{u}_{n, k+1} \neq 0$, otherwise (4.2) is trivial; equation (4.3) provides

$$
d \mathcal{F}_{n}\left(t_{n, k+1}, u_{n, k+1}\right)\left[\dot{u}_{n, k+1} /\left\|\dot{u}_{n, k+1}\right\|_{L^{2}}\right]+\left\|\dot{u}_{n, k+1}\right\|_{L^{2}}=0 .
$$

Hence, choosing $z=\dot{u}_{n, k+1} /\left\|\dot{u}_{n, k+1}\right\|_{L^{2}}$ in (4.5) gives $\left|\partial \mathcal{F}_{n}\right|_{L_{+}^{2}}\left(t_{n, k}, u_{n, k+1}\right)=$ $\left\|\dot{u}_{n, k+1}\right\|_{L^{2}}$ and the last equality in (4.2) is proved.

Lemma 4.4. For every $0 \leq k \leq n-1$, the following energy estimate holds

$$
\begin{aligned}
\mathcal{E}\left(u_{n}\left(t_{n, k+1}\right)\right)= & \mathcal{E}\left(u_{n}\left(t_{n, k}\right)\right)-\int_{t_{n, k}}^{t_{n, k+1}} \frac{1}{2}\left|\dot{u}_{n}(t)\right|_{L_{+}^{2}}^{2}+\frac{1}{2}\left|\partial \mathcal{F}_{n}\right|_{L_{+}^{2}}^{2}\left(t, u_{n}^{\sharp}(t)\right) d t+ \\
& +\int_{t_{n, k}}^{t_{n, k+1}}\left\langle f_{n}(t), \dot{u}_{n}(t)\right\rangle d t .
\end{aligned}
$$

Proof. Using (4.2) and being $\mathcal{F}_{n}\left(t_{n, k+1}, \cdot\right)$ convex we get, for $t \in\left(t_{n, k}, t_{n, k+1}\right)$,

$$
\begin{aligned}
\mathcal{F}_{n}\left(t_{n, k+1}, u_{n, k}\right) \geq & \mathcal{F}_{n}\left(t_{n, k+1}, u_{n, k+1}\right)+d \mathcal{F}_{n}\left(t_{n, k+1}, u_{n, k+1}\right)\left[u_{n, k}-u_{n, k+1}\right] \\
= & \mathcal{F}_{n}\left(t_{n, k+1}, u_{n, k+1}\right)-\tau_{n} d \mathcal{F}_{n}\left(t_{n, k+1}, u_{n, k+1}\right)\left[\dot{u}_{n}(t)\right] \\
= & \mathcal{F}_{n}\left(t_{n, k+1}, u_{n, k+1}\right)+\tau_{n}\left(\frac{1}{2}\left|\dot{u}_{n}(t)\right|_{L_{+}^{2}}^{2}\right. \\
& \left.+\frac{1}{2}\left|\partial \mathcal{F}_{n}\right|_{L_{+}^{2}}^{2}\left(t_{n, k+1}, u_{n, k+1}\right)\right) .
\end{aligned}
$$

Writing

$$
\begin{aligned}
\mathcal{F}_{n}\left(t_{n, k+1}, u_{n, k}\right) & =\mathcal{E}\left(u_{n, k}\right)-\left\langle f_{n, k}, u_{n, k}\right\rangle, \\
\mathcal{F}_{n}\left(t_{n, k+1}, u_{n, k+1}\right) & =\mathcal{E}\left(u_{n, k+1}\right)-\left\langle f_{n, k}, u_{n, k+1}\right\rangle,
\end{aligned}
$$

we get 


$$
\begin{aligned}
\mathcal{E}\left(u_{n, k}\right)-\left\langle f_{n, k}, u_{n, k}\right\rangle \geq & \mathcal{E}\left(u_{n, k+1}\right)-\left\langle f_{n, k}, u_{n, k+1}\right\rangle \\
& +\tau_{n}\left(\frac{1}{2}\left|\dot{u}_{n}(t)\right|_{L_{+}^{2}}^{2}+\frac{1}{2}\left|\partial \mathcal{F}_{n}\right|_{L_{+}^{2}}^{2}\left(t_{n, k+1}, u_{n, k+1}\right)\right) .
\end{aligned}
$$

Using the interpolation $u_{n}, u_{n}^{\sharp}$, and $f_{n}$ we get (4.6).

Proof of Proposition 4.1. Using (4.6) for $0 \leq k \leq n-1$ and (4.2) we get

$$
\mathcal{E}\left(u_{n}(T)\right) \leq \mathcal{E}\left(u_{0}\right)-\int_{0}^{T}\left\|\dot{u}_{n}(t)\right\|_{L^{2}}^{2}+\int_{0}^{T}\left\langle f_{n}(t), \dot{u}_{n}(t)\right\rangle d t .
$$

Hence $\left\|\dot{u}_{n}\right\|_{L^{2}\left(0, T ; L^{2}\right)}^{2} \leq C+\left\|f_{n}\right\|_{L^{2}\left(0, T ; L^{2}\right)}\left\|\dot{u}_{n}\right\|_{L^{2}\left(0, T ; L^{2}\right)}$. Being $f_{n}$ bounded in $L^{2}\left(0, T ; L^{2}\right)$ the sequence $u_{n}$ turns out to be bounded in $H^{1}\left(0, T ; L^{2}\right)$ and thus, up to subsequences (not relabeled), $u_{n} \rightarrow u$ in $H^{1}\left(0, T ; L^{2}\right)$. By weak convergence $\dot{u}_{n} \geq 0$ implies $\dot{u} \geq 0$. We will identify $u$ with its absolutely continuous representative, i.e.

$$
u(t)=u_{0}+\int_{0}^{t} \dot{u}(s) d s .
$$

Hence $u_{n}(t) \rightarrow u(t)$ in $L^{2}$ for every $t \in(0, T)$. We claim that $u_{n}^{\sharp} \rightarrow u$ in $H^{1}\left(0, T ; L^{2}\right)$ and that $u_{n}^{\sharp}(t) \rightarrow u(t)$ in $H_{0}^{1}$ for every $t \in[0, T]$. For every $0 \leq k \leq n-1$ from (4.6) we get

$$
\mathcal{E}\left(u_{n}\left(t_{n, k+1}\right)\right) \leq \mathcal{E}\left(u_{0}\right)+\int_{0}^{t_{n, k+1}}\left\langle f_{n}(t), \dot{u}_{n}(t)\right\rangle d t .
$$

By coercivity of the stored elastic energy, we deduce that $u_{n}$ is bounded in $L^{\infty}\left(0, T ; H_{0}^{1}\right)$ and thus $u_{n}^{\sharp}$ is bounded in $L^{\infty}\left(0, T ; H_{0}^{1}\right)$ as well. Given $t \in(0, T]$ let $k_{n}$ such that $t_{n, k_{n}}<t \leq t_{n, k_{n}+1}$. Remembering that $u_{n}^{\sharp}(t)=u_{n}\left(t_{n, k_{n}+1}\right)$ we can write

$$
\begin{aligned}
\left\|u_{n}^{\sharp}(t)-u_{n}(t)\right\|_{L^{2}} & =\left\|u_{n}\left(t_{n, k_{n}+1}\right)-u_{n}(t)\right\|_{L^{2}}=\left\|\int_{t}^{t_{n, k_{n}+1}} \dot{u}_{n}(s) d s\right\|_{L^{2}} \\
& \leq \int_{t}^{t_{n, k_{n}+1}}\left\|\dot{u}_{n}(s)\right\|_{L^{2}} d s \leq\left|t_{n, k_{n}+1}-t\right|^{1 / 2}\left\|\dot{u}_{n}\right\|_{L^{2}\left(0, T ; L^{2}\right)} .
\end{aligned}
$$

It follows that $u_{n}^{\sharp}(t) \rightarrow u(t)$ in $L^{2}$ for every $t \in[0, T]$. As $u_{n}^{\sharp}(t)$ is bounded in $H_{0}^{1}$ it follows that $u_{n}^{\sharp}(t) \rightarrow u(t)$ in $H_{0}^{1}$. get

Given $t^{*} \in(0, T]$ let $k_{n}$ such that $t_{n, k_{n}}<t^{*} \leq t_{n, k_{n}+1}$. Using (4.6) we

$$
\begin{aligned}
& \mathcal{E}\left(u_{n}\left(t_{n, k_{n}+1}\right)\right)+\int_{0}^{t_{n, k_{n}+1}} \frac{1}{2}\left|\dot{u}_{n}(t)\right|_{L_{+}^{2}}^{2}+\frac{1}{2}\left|\partial \mathcal{F}_{n}\right|_{L_{+}^{2}}^{2}\left(t, u_{n}^{\sharp}(t)\right) d t \\
& -\int_{0}^{t_{n, k_{n}+1}}\left\langle f_{n}(t), \dot{u}_{n}(t)\right\rangle d t \leq \mathcal{E}\left(u_{0}\right) .
\end{aligned}
$$

Clearly $t_{n, k_{n}+1} \rightarrow t^{*}$ and the sequence $u_{n, k_{n}+1}=u_{n}^{\sharp}\left(t^{*}\right)$ converges weakly to $u\left(t^{*}\right)$ in $H_{0}^{1}$. Hence

$$
\mathcal{E}\left(u\left(t^{*}\right)\right) \leq \liminf _{n \rightarrow+\infty} \mathcal{E}\left(u_{n}\left(t_{n, k_{n}+1}\right)\right)
$$


By weak convergence in $H^{1}\left(0, T ; L^{2}\right)$ we get

$$
\begin{aligned}
\int_{0}^{t^{*}}\|\dot{u}(t)\|_{L^{2}}^{2} d t & \leq \liminf _{n \rightarrow+\infty} \int_{0}^{t^{*}}\left\|\dot{u}_{n}(t)\right\|_{L^{2}}^{2} d t \\
& \leq \liminf _{n \rightarrow+\infty} \int_{0}^{t_{n, k_{n}+1}}\left\|\dot{u}_{n}(t)\right\|_{L^{2}}^{2} d t .
\end{aligned}
$$

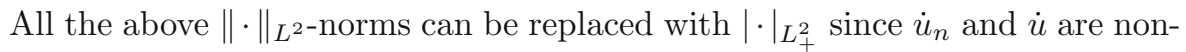
negative. Since $f_{n}(t) \rightarrow f(t)$ in $L^{2}$ and $u_{n}^{\sharp}(t) \rightarrow u(t)$ in $H^{1}$ for a.e. $t \in(0, T)$, by (3.3) we get

$$
|\partial \mathcal{F}|_{L_{+}^{2}}(t, u(t)) \leq \liminf _{n \rightarrow+\infty}\left|\partial \mathcal{F}_{n}\right|_{L_{+}^{2}}\left(t, u_{n}^{\sharp}(t)\right) .
$$

Then, by Fatou's Lemma

$$
\begin{aligned}
\int_{0}^{t^{*}}|\partial \mathcal{F}|_{L_{+}^{2}}^{2}(t, u(t)) d t & \leq \liminf _{n \rightarrow+\infty} \int_{0}^{t^{*}}\left|\partial \mathcal{F}_{n}\right|_{L_{+}^{2}}^{2}\left(t, u_{n}^{\sharp}(t)\right) d t \\
& \leq \liminf _{n \rightarrow+\infty} \int_{0}^{t_{n, k_{n}+1}}\left|\partial \mathcal{F}_{n}\right|_{L_{+}^{2}}^{2}\left(t, u_{n}^{\sharp}(t)\right) d t .
\end{aligned}
$$

For last term in the left hand side of (4.8) we easily have

$$
\lim _{n \rightarrow+\infty} \int_{0}^{t^{*}}\left\langle f_{n}(t), \dot{u}_{n}(t)\right\rangle d t=\int_{0}^{t^{*}}\langle f(t), \dot{u}(t)\rangle d t .
$$

Taking the liminf in (4.8) gives the thesis.

\subsection{Unconstrained incremental problem with a posteriori truncation}

In this section we consider an alternative discrete scheme, numerically more convenient than (4.1), employed in $[3,26]$. Let $\tau_{n}$ and $t_{n, k}$ be as in the previous subsection. Define $u_{n, 0}=u_{0}$ at time $t_{n, 0}$ and then by induction let

$$
\left\{\begin{array}{l}
\tilde{u}_{n, k+1} \in \operatorname{argmin}\left\{\mathcal{F}_{n}\left(t_{n, k+1}, u\right)+\frac{1}{2 \tau_{n}}\left\|u-u_{n, k}\right\|_{L^{2}}^{2}: u \in H_{0}^{1}\right\} \\
u_{n, k+1}=\max \left\{\tilde{u}_{n, k+1}, u_{n, k}\right\} .
\end{array}\right.
$$

Note that the first minimization is unconstrained, the constraint is taken into account a posteriori, simply by truncation. In this way $u_{n, k+1}-u_{n, k}=\left[\tilde{u}_{n, k+1}-\right.$ $\left.u_{n, k}\right]_{+}$.

As in the previous subsection, $u_{n}:[0, T] \rightarrow L^{2}$ denotes the piecewise affine interpolation and $u_{n}^{\sharp}$ denotes the piecewise constant backward (leftcontinuous) interpolation of $u_{n, k}$ in the points $t_{n, k}$. Moreover, we introduce $\tilde{u}_{n}:[0, T] \rightarrow L^{2}$, the piecewise constant backwards (left-continuous) interpolation of $\tilde{u}_{n, k}$.

Proposition 4.5. Upon extracting a subsequence (not relabeled) $u_{n} \rightarrow u$ in $H^{1}\left(0, T ; L^{2}\right)$ where $u$ is a unilateral gradient flow in the sense of Definition 2.2.

Lemma 4.6. For every index $0 \leq k \leq n-1$

$$
\left|\dot{u}_{n}(t)\right|_{L_{+}^{2}}=\left\|\dot{u}_{n}(t)\right\|_{L^{2}}=\left|\partial \mathcal{F}_{n}\right|_{L_{+}^{2}}\left(t, \tilde{u}_{n}(t)\right) \quad \text { for } t \in\left(t_{n, k}, t_{n, k+1}\right) .
$$

Moreover, 


$$
\begin{aligned}
& d \mathcal{F}_{n}\left(t_{n, k+1}, u_{n, k+1}\right)\left[u_{n, k+1}-u_{n, k}\right] \\
& \quad=d \mathcal{F}_{n}\left(t_{n, k+1}, \tilde{u}_{n, k+1}\right)\left[u_{n, k+1}-u_{n, k}\right] \\
& \quad=-\left|\partial \mathcal{F}_{n}\right|_{L_{+}^{2}}\left(t_{n, k+1}, \tilde{u}_{n, k+1}\right)\left\|u_{n, k+1}-u_{n, k}\right\|_{L^{2}}
\end{aligned}
$$

Proof. By minimality

$$
d \mathcal{F}_{n}\left(t_{n, k+1}, \tilde{u}_{n, k+1}\right)[z]+\frac{1}{\tau_{n}}\left\langle\tilde{u}_{n, k+1}-u_{n, k}, z\right\rangle=0 \quad \text { for every } z \in H_{0}^{1} .
$$

Hence, by (3.2)

$$
\begin{aligned}
& \left|\partial \mathcal{F}_{n}\right|_{L_{+}^{2}}\left(t_{n, k+1}, \tilde{u}_{n, k+1}\right) \\
& \quad=\sup \left\{-d \mathcal{F}_{n}\left(t_{n, k+1}, \tilde{u}_{n, k+1}\right)[z]: z \in H_{0}^{1}, z \geq 0,\|z\|_{L^{2}} \leq 1\right\} \\
& \quad=\max \left\{\frac{1}{\tau_{n}}\left\langle\tilde{u}_{n, k+1}-u_{n, k}, z\right\rangle: z \in L^{2}, z \geq 0,\|z\|_{L^{2}} \leq 1\right\} \\
& \quad=\frac{1}{\tau_{n}}\left\langle\tilde{u}_{n, k+1}-u_{n, k}, \frac{\left[\tilde{u}_{n, k+1}-u_{n, k}\right]_{+}}{\left\|\left[\tilde{u}_{n, k+1}-u_{n, k}\right]_{+}\right\|_{L^{2}}}\right\rangle \\
& \quad=\frac{1}{\tau_{n}}\left\|\left[\tilde{u}_{n, k+1}-u_{n, k}\right]_{+}\right\|_{L^{2}}=\frac{1}{\tau}\left|u_{n, k+1}-u_{n, k}\right|_{L_{+}^{2}},
\end{aligned}
$$

which gives (4.13). In particular, remembering that $u_{n, k+1}-u_{n, k}=\left[\tilde{u}_{n, k+1}-\right.$ $\left.u_{n, k}\right]_{+}$we get

$$
\begin{aligned}
& -d \mathcal{F}_{n}\left(t_{n, k+1}, \tilde{u}_{n, k+1}\right)\left[u_{n, k+1}-u_{n, k}\right] \\
& \quad=\left|\partial \mathcal{F}_{n}\right|_{L_{+}^{2}}\left(t_{n, k+1}, \tilde{u}_{n, k+1}\right)\left\|u_{n, k+1}-u_{n, k}\right\|_{L^{2}}
\end{aligned}
$$

which gives (4.15).

It remains to prove (4.14). Define the sets $\Omega_{-}=\left\{\tilde{u}_{n, k+1} \leq u_{n, k}\right\}$ and $\Omega_{+}=\left\{\tilde{u}_{n, k+1}>u_{n, k}\right\}$. We claim that

$$
d \mathcal{F}_{n}\left(t_{n, k+1}, u_{n, k+1}\right)[z]+\frac{1}{\tau_{n}}\left\langle u_{n, k+1}-u_{n, k}, z\right\rangle=0
$$

for every $z \in H_{0}^{1}$ with $z=0$ in $\Omega_{-}$. Since $z=0$ in $\Omega_{-}$

$$
\int_{\Omega_{-}} \nabla u_{n, k+1} \cdot B \nabla z+b u_{n, k+1} z-f_{n, k+1} z+\frac{1}{\tau_{n}}\left(u_{n, k+1}-u_{n, k}\right) z d x=0 .
$$

As $\tilde{u}_{n, k+1}=u_{n, k+1}$ in $\Omega_{+}$and $z=0$ in $\Omega_{-}$

$$
\begin{aligned}
\int_{\Omega_{+}} & \nabla u_{n, k+1} \cdot B \nabla z+b u_{n, k+1} z-f_{n, k+1} z+\frac{1}{\tau_{n}}\left(u_{n, k+1}-u_{n, k}\right) z d x \\
= & \int_{\Omega_{+}} \nabla \tilde{u}_{n, k+1} \cdot B \nabla z+b \tilde{u}_{n, k+1} z-f_{n, k+1} z+\frac{1}{\tau_{n}}\left(\tilde{u}_{n, k+1}-u_{n, k}\right) z d x \\
= & \int_{\Omega} \nabla \tilde{u}_{n, k+1} \cdot B \nabla z+b \tilde{u}_{n, k+1} z-f_{n, k+1} z+\frac{1}{\tau_{n}}\left(\tilde{u}_{n, k+1}-u_{n, k}\right) z d x=0,
\end{aligned}
$$

where the last equality follows by minimality. Joining the integrals on $\Omega^{ \pm}$ proves (4.18). we get

Using (4.16) and (4.18) with $z=u_{n, k+1}-u_{n, k}$ (note that $z=0$ in $\Omega_{-}$) 


$$
\begin{aligned}
& d \mathcal{F}_{n}\left(t_{n, k+1}, \tilde{u}_{n, k+1}\right)\left[u_{n, k+1}-u_{n, k}\right]+\frac{1}{\tau_{n}}\left\langle\tilde{u}_{n, k+1}-u_{n, k}, u_{n, k+1}-u_{n, k}\right\rangle=0 \\
& d \mathcal{F}_{n}\left(t_{n, k+1}, u_{n, k+1}\right)\left[u_{n, k+1}-u_{n, k}\right]+\frac{1}{\tau_{n}}\left\langle u_{n, k+1}-u_{n, k}, u_{n, k+1}-u_{n, k}\right\rangle=0 .
\end{aligned}
$$

Let us see that $\left\langle\tilde{u}_{n, k+1}-u_{n, k}, u_{n, k+1}-u_{n, k}\right\rangle=\left\langle u_{n, k+1}-u_{n, k}, u_{n, k+1}-u_{n, k}\right\rangle$, indeed, their difference reads $\left\langle\tilde{u}_{n, k+1}-u_{n, k+1}, u_{n, k+1}-u_{n, k}\right\rangle$ which vanishes because $\tilde{u}_{n, k+1}-u_{n, k+1}=0$ in $\Omega_{+}$and $u_{n, k+1}-u_{n, k}=0$ in $\Omega_{-}$. Hence $d \mathcal{F}_{n}\left(t_{n, k+1}, \tilde{u}_{n, k+1}\right)\left[u_{n, k+1}-u_{n, k}\right]=d \mathcal{F}_{n}\left(t_{n, k+1}, u_{n, k+1}\right)\left[u_{n, k+1}-u_{n, k}\right]$.

Next lemma follows closely the corresponding one in the previous subsection.

Lemma 4.7. For every $0 \leq k \leq n-1$, the following energy estimate holds

$$
\begin{aligned}
\mathcal{E}\left(u_{n}\left(t_{n, k+1}\right)\right) \leq & \mathcal{E}\left(u_{n}\left(t_{n, k}\right)\right)-\int_{t_{n, k}}^{t_{n, k+1}} \frac{1}{2}\left|\dot{u}_{n}(t)\right|_{L_{+}^{2}}^{2}+\frac{1}{2}\left|\partial \mathcal{F}_{n}\right|_{L_{+}^{2}}^{2}\left(t, \tilde{u}_{n}(t)\right) d t \\
& +\int_{t_{n, k+1}}^{t_{n, k+1}}\left\langle f_{n}(t), \dot{u}_{n}(t)\right\rangle d t
\end{aligned}
$$

Proof. Write, $u_{n, k+1}=u_{n}\left(t_{n, k+1}\right)$ etc. By convexity and by (4.15) and (4.13)

$$
\begin{aligned}
\mathcal{F}_{n}\left(t_{n, k+1}, u_{n, k}\right) \geq & \mathcal{F}_{n}\left(t_{n, k+1}, u_{n, k+1}\right)+d \mathcal{F}_{n}\left(t_{n, k+1}, u_{n, k+1}\right)\left[u_{n, k}-u_{n, k+1}\right] \\
\geq & \mathcal{F}_{n}\left(t_{n, k+1}, u_{n, k+1}\right)+\tau_{n}\left|\partial \mathcal{F}_{n}\right|_{L_{+}^{2}}\left(t_{n, k+1}, \tilde{u}_{n, k+1}\right)\left\|\dot{u}_{n}\right\|_{L^{2}} \\
\geq & \mathcal{F}_{n}\left(t_{n, k+1}, u_{n, k+1}\right) \\
& +\tau_{n}\left(\frac{1}{2}\left\|\dot{u}_{n}\right\|_{L^{2}}^{2}+\frac{1}{2}\left|\partial \mathcal{F}_{n}\right|_{L_{+}^{2}}^{2}\left(t_{n, k+1}, \tilde{u}_{n, k+1}\right)\right) .
\end{aligned}
$$

Following line by line the proof of Lemma 4.4 provides (4.19).

Note that formally (since the sequences do not coincide) the only difference between (4.6) and (4.19) is the slope: in the former it is evaluated in $\left(t, u_{n}^{\sharp}(t)\right)$ while in the latter in $\left(t, \tilde{u}_{n}(t)\right)$.

Proof of Proposition 4.5. Following line by line the first step in the proof of Proposition 4.1 we get that the sequence $u_{n}$ is bounded in $L^{\infty}\left(0, T ; H_{0}^{1}\right)$ and in $H^{1}\left(0, T ; L^{2}\right)$. Hence $u_{n} \rightarrow u$ in $H^{1}\left(0, T ; L^{2}\right)$, upon extracting a (non-relabeled) subsequence.

We claim that $\tilde{u}_{n}(t) \rightarrow u(t)$ in $H_{0}^{1}$ for a.e. $t \in(0, T)$. Fix $t \in(0, T)$ such that $f_{n}(t) \rightarrow f(t)$ in $L^{2}$. For every $n \in \mathbb{N}$ let $k_{n}$ such that $t_{n, k_{n}}<t \leq t_{n, k_{n}+1}$. Note that $f_{n, k+1}=f_{n}(t) \rightarrow f(t)$ in $L^{2}$. By minimality we can write that

$$
\begin{aligned}
& \mathcal{F}_{n}\left(t_{n, k+1}, \tilde{u}_{n, k+1}\right)+\frac{1}{2 \tau_{n}}\left\|\tilde{u}_{n, k+1}-u_{n, k}\right\|_{L^{2}}^{2} \leq \mathcal{F}_{n}\left(t_{n, k+1}, u_{n, k}\right) \\
& \quad \leq c\left\|u_{n, k}\right\|_{H_{0}^{1}}^{2}+\left\|f_{n, k+1}\right\|_{L^{2}}\left\|u_{n, k}\right\|_{L^{2}},
\end{aligned}
$$

where we used the continuity of the bi-linear form. Since $u_{n}$ is bounded in $L^{\infty}\left(0, T ; H_{0}^{1}\right)$ the right hand side is bounded uniformly with respect to $n$ and $k$; thus there exists $C>0$ s.t.

$$
\begin{aligned}
& \left\|\tilde{u}_{n, k+1}-u_{n, k}\right\|_{L^{2}}^{2} \leq C \tau_{n}, \\
& c^{\prime}\left\|\tilde{u}_{n, k+1}\right\|_{H_{0}^{1}}^{2}-\left\|f_{n, k+1}\right\|_{L^{2}}\left\|\tilde{u}_{n, k+1}\right\|_{H_{0}^{1}} \leq \mathcal{F}_{n}\left(t_{n, k+1}, \tilde{u}_{n, k+1}\right) \leq C,
\end{aligned}
$$


where in the second line we used coercivity. As $f_{n, k+1} \rightarrow f(t)$ in $L^{2}$, a simple algebraic estimate yields $\left\|\tilde{u}_{n, k+1}\right\|_{H_{0}^{1}} \leq C^{\prime}$.

Since $u_{n, k}=u_{n}\left(t_{n, k}\right) \rightarrow u(t)$ in $L^{2}$ it follows that $\tilde{u}_{n}(t)=\tilde{u}_{n, k+1} \rightarrow u(t)$ in $L^{2}$. Being $\tilde{u}_{n, k+1}$ bounded uniformly in $H_{0}^{1}$ we get $\tilde{u}_{n}(t) \rightarrow u(t)$ in $H_{0}^{1}$.

To conclude the proof it is enough to argue as in the proof of Proposition 4.1

\subsection{Unconstrained incremental problem with penalty}

Let $\alpha>1$. For $v \in L^{2}$ let us denote

$$
\psi_{\alpha}(v)=\left\{\begin{array}{ll}
v^{2} & \text { if } v \geq 0 \\
\alpha v^{2} & \text { if } v<0,
\end{array} \quad \Psi_{\alpha}(v)=\int_{\Omega} \psi_{\alpha}(v) d x, \quad|v|_{L_{\alpha}^{2}}=\Psi_{\alpha}^{1 / 2}(v) .\right.
$$

Clearly, when $\alpha$ is large $|v|_{L_{\alpha}^{2}}$ penalizes $v_{-}$. Note that $\Psi_{\alpha}$ can be equivalently seen as a Yosida regularization of the indicator function of the set $\left\{v \in L^{2}\right.$ : $v \geq 0\}$.

Moreover, we have $|v|_{L_{\alpha}^{2}} \geq\|v\|_{L^{2}} \geq|v|_{L_{\alpha}^{2}} / \alpha$, hence $\left|v_{n}-v\right|_{L_{\alpha}^{2}} \rightarrow 0$ is equivalent to $v_{n} \rightarrow v$ in $L^{2}$. Clearly

$$
d \Psi_{\alpha}(v)[z]=\int_{\Omega} \psi_{\alpha}^{\prime}(v) z d x=2 \int_{\{v>0\}} v z d x+2 \alpha \int_{\{v<0\}} v z d x .
$$

Before proceeding, let us prove this lemma.

Lemma 4.8. For $v \in L^{2}$ it holds

$$
\begin{aligned}
\sup \left\{d \Psi_{\alpha}(v)[z]: z \in H_{0}^{1},|z|_{L_{\alpha}^{2}} \leq 1\right\} & =\max \left\{d \Psi_{\alpha}(v)[z]: z \in L^{2},|z|_{L_{\alpha}^{2}} \leq 1\right\} \\
& =2|v|_{L_{\alpha}^{2}} .
\end{aligned}
$$

Proof. We introduce the set $\hat{V}=\left\{z \in L^{2}: z=0\right.$ if $v=0, z \geq 0$ if $v>0, z \leq$ 0 if $v<0\}$ and we will prove that

$$
\begin{aligned}
\sup \left\{d \Psi_{\alpha}(v)[z]: z \in H_{0}^{1},|z|_{L_{\alpha}^{2}} \leq 1\right\} & =\sup \left\{d \Psi_{\alpha}(v)[z]: z \in L^{2},|z|_{L_{\alpha}^{2}} \leq 1\right\} \\
& =\sup \left\{d \Psi_{\alpha}(v)[z]: z \in \hat{V},|z|_{L_{\alpha}^{2}} \leq 1\right\} \\
& =2|v|_{L_{\alpha}^{2}} .
\end{aligned}
$$

It is enough to consider $|v|_{L_{\alpha}^{2}} \neq 0$, otherwise there is nothing to prove. The first identity follows by density of $H_{0}^{1}$ in $L^{2}$ and by continuity of $|\cdot|_{L_{\alpha}^{2}}$ and $d \Psi_{\alpha}(v)[\cdot]$. Let us check the second identity. Clearly

$$
\sup \left\{d \Psi_{\alpha}(v)[z]: z \in L^{2},|z|_{L_{\alpha}^{2}} \leq 1\right\} \geq \sup \left\{d \Psi_{\alpha}(v)[z]: z \in \hat{V},|z|_{L_{\alpha}^{2}} \leq 1\right\} .
$$

In order to prove the opposite inequality, given $z \in L^{2}$ let $\hat{z} \in L^{2}$ be defined by

$$
\hat{z}= \begin{cases}z & \text { if } v>0 \text { and } z>0 \\ 0 & \text { if } v>0 \text { and } z \leq 0 \\ 0 & \text { if } v=0 \\ z & \text { if } v<0 \text { and } z<0 \\ 0 & \text { if } v<0 \text { and } z \geq 0\end{cases}
$$


Then, $\hat{z} \in \hat{V},|\hat{z}|_{L_{\alpha}^{2}} \leq|z|_{L_{\alpha}^{2}} \leq 1$ and $d \Psi_{\alpha}(v)[z] \leq d \Psi_{\alpha}(v)[\hat{z}]$ because

$$
\int_{\{v>0\}} v z d x \leq \int_{\{v>0\}} v \hat{z} d x \text { and } \int_{\{v<0\}} v z d x \leq \int_{\{v<0\}} v \hat{z} d x .
$$

To prove the last equality in (4.21), given $\tau$ and $v$, let us introduce the Hilbert space $L_{\lambda}^{2}$ where $\lambda$ is the measure $\lambda=\mathcal{L}_{\mid\{v \geq 0\}}^{d}+\alpha \mathcal{L}_{\mid\{v<0\}}^{d}$ (being $\mathcal{L}^{d}$ the $d$-dimensional Lebesgue measure). Then we write

$$
\begin{aligned}
d \Psi_{\alpha}(v)[z] & =2 \int_{\{v \geq 0\}} v z d x+2 \alpha \int_{\{v<0\}} v z d x \\
& =2 \int_{\Omega} v z d \lambda=2\langle v, z\rangle_{L_{\lambda}^{2}} \leq 2\|v\|_{L_{\lambda}^{2}}\|z\|_{L_{\lambda}^{2}} .
\end{aligned}
$$

Since $v$ and $z$ belong to $\hat{V}$ we have $\|v\|_{L_{\lambda}^{2}}=|v|_{L_{\alpha}^{2}}$ and $\|z\|_{L_{\lambda}^{2}}=|z|_{L_{\alpha}^{2}}$; thus the previous inequality yields

$$
\sup \left\{d \Psi_{\alpha}(v)[z]: z \in \hat{V},|z|_{L_{\alpha}^{2}} \leq 1\right\} \leq 2|v|_{L_{\alpha}^{2}} .
$$

Clearly, $z=v /|v|_{L_{\alpha}^{2}}$ is the maximizer, which gives (4.20).

Finally, let us introduce the slope

$$
\begin{aligned}
|\partial \mathcal{F}|_{L_{\alpha}^{2}}(t, u) & =\limsup _{v \rightarrow u} \frac{[\mathcal{F}(t, v)-\mathcal{F}(t, u)]_{-}}{|v-u|_{L_{\alpha}^{2}}} \\
& =\sup \left\{-\partial \mathcal{F}(t, u)[\phi]: \phi \in H_{0}^{1},|\phi|_{L_{\alpha}^{2}} \leq 1\right\},
\end{aligned}
$$

where $v \rightarrow u$ in $L_{\alpha}^{2}$, i.e., $|v-u|_{L_{\alpha}^{2}} \rightarrow 0$, and the second identity follows by the same arguments of Lemma 3.1. We remark that, if $|\phi|_{L_{+}^{2}} \leq 1$, then $\phi \geq 0$ and $\|\phi\|_{L^{2}} \leq 1$, thus $|\phi|_{L_{\alpha}^{2}}=\|\phi\|_{L^{2}} \leq 1$, hence $|\partial \mathcal{F}|_{L_{\alpha}^{2}}(t, u) \geq|\partial \mathcal{F}|_{L_{+}^{2}}(t, u)$.

Let $\alpha_{n}$ be a monotone increasing sequence with $\alpha_{0} \geq 1$ and with $\lim _{n \rightarrow+\infty} \alpha_{n}=+\infty$. Let $t_{n, k}$ and $\mathcal{F}_{n}$ be as in the previous sections. Define $u_{n, 0}=u_{0}$ and by induction

$$
u_{n, k+1} \in \operatorname{argmin}\left\{\mathcal{F}_{n}\left(t_{n, k+1}, u\right)+\frac{1}{2 \tau_{n}}\left|u-u_{n, k}\right|_{L_{\alpha_{n}}^{2}}^{2}: u \in H_{0}^{1}\right\} .
$$

Let $u_{n}:[0, T] \rightarrow L^{2}(\Omega)$ and $u_{n}^{\sharp}:[0, T] \rightarrow L^{2}(\Omega)$ denote respectively the piecewise affine and the piecewise constant interpolation of $u_{n, k}$ in $t_{n, k}$, as in the previous sections.

Proposition 4.9. Upon extracting a subsequence (non relabeled) $u_{n} \rightarrow u$ in $H^{1}\left(0, T ; L^{2}\right)$ where $u$ is a unilateral gradient flow, in the sense of Definition 2.2.

Lemma 4.10. For every $t \in\left(t_{n, k}, t_{n, k+1}\right)$

$$
\left.\left|\dot{u}_{n}(t)\right|_{L_{\alpha_{n}}^{2}}^{2}=-d \mathcal{F}_{n}\left(t_{n, k+1}, u_{n, k+1}\right)\right)\left[\dot{u}_{n}(t)\right]=\left|\partial \mathcal{F}_{n}\right|_{L_{\alpha_{n}}^{2}}^{2}\left(t, u_{n}^{\sharp}(t)\right) .
$$

Proof. By minimality $u_{n, k+1}$ satisfies the Euler-Lagrange equation

$$
d \mathcal{F}_{n}\left(t_{n, k+1}, u_{n, k+1}\right)[z]+\frac{1}{2} d \Psi_{\alpha_{n}}\left(\dot{u}_{n}\right)[z]=0 \quad \text { for every } z \in H_{0}^{1} .
$$

Hence, by Lemma 4.8 


$$
\begin{aligned}
& \left|\partial \mathcal{F}_{n}\right|_{L_{\alpha_{n}}^{2}}\left(t_{n, k+1}, u_{n, k+1}\right) \\
& \quad=\sup \left\{-d \mathcal{F}_{n}\left(t_{n, k+1}, u_{n, k+1}\right)[z]: z \in H_{0}^{1},|z|_{L_{\alpha_{n}}^{2}} \leq 1\right\} \\
& \quad=\sup \left\{\frac{1}{2} d \Psi_{\alpha_{n}}\left(\dot{u}_{n}\right)[z]: z \in H_{0}^{1},|z|_{L_{\alpha_{n}}^{2}} \leq 1\right\}=\left|\dot{u}_{n}\right|_{L_{\alpha_{n}}^{2}} .
\end{aligned}
$$

By Lemma 4.10, choosing $z=\dot{u}_{n}$ we obtain

$$
\begin{aligned}
-d \mathcal{F}_{n}\left(t_{n, k+1}, u_{n, k+1}\right)\left[\dot{u}_{n}\right] & =\frac{1}{2} d \Psi_{\alpha_{n}}\left(\dot{u}_{n}\right)\left[\dot{u}_{n}\right] \\
& =\left|\dot{u}_{n}\right|_{L_{\alpha_{n}}^{2}}^{2}=\left|\partial \mathcal{F}_{n}\right|_{L_{\alpha_{n}}^{2}}^{2}\left(t_{n, k+1}, u_{n, k+1}\right),
\end{aligned}
$$

which concludes the proof.

Lemma 4.11. For every $0 \leq k \leq n-1$, the following energy estimate holds

$$
\begin{aligned}
\mathcal{E}\left(u_{n}\left(t_{n, k+1}\right)\right) \leq \mathcal{E} & \left(u_{n}\left(t_{n, k}\right)\right)-\int_{t_{n, k}}^{t_{n, k+1}} \frac{1}{2}\left|\dot{u}_{n}(t)\right|_{L_{\alpha_{n}}^{2}}^{2}+\frac{1}{2}\left|\partial \mathcal{F}_{n}\right|_{L_{\alpha_{n}}^{2}}^{2}\left(t, u_{n}^{\sharp}(t)\right) d t \\
& +\int_{t_{n, k}}^{t_{n, k+1}}\left\langle f_{n}(t), \dot{u}_{n}(t)\right\rangle d t .
\end{aligned}
$$

Proof. It is enough to combine the proof of Lemma 4.4 with Lemma 4.10.

Proof of Proposition 4.9. As $\left|\dot{u}_{n}\right|_{L_{\alpha_{n}}^{2}} \geq\left\|\dot{u}_{n}\right\|_{L^{2}}$, arguing as in the proof of Proposition 4.1, it follows that the sequence $u_{n}$ is bounded in $H^{1}\left(0, T ; L^{2}\right)$ and in $L^{\infty}\left(0, T ; H_{0}^{1}\right)$. Thus, upon extracting a subsequence (non relabeled) $u_{n} \rightarrow u$ in $H^{1}\left(0, T ; L^{2}\right)$ and $u_{n} \stackrel{*}{\rightarrow} u$ in $L^{\infty}\left(0, T ; H_{0}^{1}\right)$.

Given $t^{*} \in(0, T]$ let $k_{n}$ such that $t_{n, k_{n}}<t^{*} \leq t_{n, k_{n}+1}$; by the previous lemma we have

$$
\begin{aligned}
& \mathcal{E}\left(u_{n}\left(t_{n, k_{n}+1}\right)\right)+\int_{0}^{t_{n, k_{n}+1}} \frac{1}{2}\left|\dot{u}_{n}(t)\right|_{L_{\alpha_{n}}^{2}}^{2}+\frac{1}{2}\left|\partial \mathcal{F}_{n}\right|_{L_{\alpha_{n}}^{2}}^{2}\left(t, u_{n}^{\sharp}(t)\right) d t \\
& \quad+\int_{0}^{t_{n, k_{n}+1}}\left\langle f_{n}(t), \dot{u}_{n}(t)\right\rangle d t \leq \mathcal{E}\left(u_{0}\right) .
\end{aligned}
$$

As in the proof of Proposition 4.1 we get that $u_{n}^{\sharp}(t) \rightarrow u(t)$ in $H_{0}^{1}$ and thus by convexity of the energy $\mathcal{E}\left(u\left(t^{*}\right)\right) \leq \liminf _{n \rightarrow+\infty} \mathcal{E}\left(u_{n}\left(t_{n, k_{n}+1}\right)\right)$.

Note that $\left|\dot{u}_{n}\right|_{L_{\alpha_{n}}^{2}} \geq\left|\dot{u}_{n}\right|_{L_{\alpha}^{2}}$ for every $\alpha_{n} \geq \alpha$ and that $|\cdot|_{L_{\alpha}^{2}}^{2}$ is positive and convex. Then, by weak convergence in $H^{1}\left(0, T ; L^{2}\right)$ for every $\tau>0$ we get

$$
\begin{aligned}
\int_{0}^{t^{*}}|\dot{u}(t)|_{L_{\alpha}^{2}}^{2} d t & \leq \liminf _{n \rightarrow+\infty} \int_{0}^{t^{*}}\left|\dot{u}_{n}(t)\right|_{L_{\alpha}^{2}}^{2} d t \\
& \leq \liminf _{n \rightarrow+\infty} \int_{0}^{t_{n, k_{n}+1}}\left|\dot{u}_{n}(t)\right|_{L_{\alpha_{n}}^{2}}^{2} d t .
\end{aligned}
$$

Note that

$$
\sup _{\alpha \in[1,+\infty)}|z|_{L_{\alpha}^{2}}=|z|_{L_{+}^{2}}= \begin{cases}\|z\|_{L^{2}} & \text { if } z \geq 0 \\ +\infty & \text { otherwise. }\end{cases}
$$

Then, taking the supremum with respect to $\alpha$ in (4.24) by monotone convergence we get

$$
\int_{0}^{t^{*}}|\dot{u}(t)|_{L_{+}^{2}}^{2} d t \leq \liminf _{n \rightarrow+\infty} \int_{0}^{t_{n, k_{n}+1}}\left|\dot{u}_{n}(t)\right|_{L_{\alpha_{n}}^{2}}^{2} d t .
$$


Since $u_{n}^{\sharp}(t) \rightarrow u(t)$ in $H_{0}^{1}$, by Corollary 3.4 together with $\left|\partial \mathcal{F}_{n}\right|_{L_{+}^{2}}(t, \cdot) \leq$ $\left|\partial \mathcal{F}_{n}\right|_{L_{\alpha}^{2}}(t, \cdot)$ we obtain

$$
|\partial \mathcal{F}|_{L_{+}^{2}}(t, u(t)) \leq \liminf _{n \rightarrow+\infty}\left|\partial \mathcal{F}_{n}\right|_{L_{+}^{2}}\left(t, u_{n}^{\sharp}(t)\right) \leq \liminf _{n \rightarrow+\infty}\left|\partial \mathcal{F}_{n}\right|_{L_{\alpha_{n}}^{2}}\left(t, u_{n}^{\sharp}(t)\right) .
$$

Passing to the limit in the discrete energy estimate, as in the proof of Proposition 4.1, we conclude the proof.

\subsection{Energy identity, uniqueness and strong convergence}

This section contains the proof of Theorem 2.3. We give first a short proof of the following Lemma.

Lemma 4.12. Let $w \in H^{1}\left(0, T ; L^{2}\right)$. Consider a sequence of finite subdivisions $t_{j, i}$ of $[0, T]$ with $0=t_{j, 0}<\ldots<t_{j, i}<t_{j, i+1}<\ldots<t_{j, I_{j}}=T$ and let $\tau_{j}=\max _{i}\left(t_{j, i+1}-t_{j, i}\right)$. Let $w_{j}$ be the piecewise affine interpolation of (the continuous representative) of $w$ in the points $t_{j, i}$. Then

$$
\begin{aligned}
& \int_{0}^{T}\left\|\dot{w}_{j}\right\|_{L^{2}}^{2} d t \leq \int_{0}^{T}\|\dot{w}\|_{L^{2}}^{2} d t \\
& \int_{0}^{T}\left\|w_{j}-w\right\|_{L^{2}}^{2} d t \leq 4 \tau_{j}^{2} \int_{0}^{T}\|\dot{w}\|_{L^{2}}^{2} d t .
\end{aligned}
$$

If $\tau_{j} \rightarrow 0$ then $w_{j} \rightarrow w$ in $H^{1}\left(0, T ; L^{2}\right)$ and $\left\|\dot{w}_{j}\right\|_{L^{2}} \rightarrow\|\dot{w}\|_{L^{2}}$ in $L^{2}(0, T)$.

Proof. For $t \in\left(t_{j, i}, t_{j, i+1}\right)$ we have $\dot{w}_{j}=f_{t_{j, i}}^{t_{j, i+1}} \dot{w}(t) d t$. Hence, by Jensen inequality

$$
\begin{aligned}
\int_{t_{j, i}}^{t_{j, i+1}}\left\|\dot{w}_{j}(t)\right\|_{L^{2}}^{2} d t & =\left(t_{j, i+1}-t_{j, i}\right)\left\|\dot{w}_{j}\right\|_{L^{2}}^{2}=\left(t_{j, i+1}-t_{j, i}\right)\left\|f_{t_{j, i}}^{t_{j, i+1}} \dot{w}(t) d t\right\|_{L^{2}}^{2} \\
& \leq \int_{t_{j, i}}^{t_{j, i+1}}\|\dot{w}(t)\|_{L^{2}}^{2} d t .
\end{aligned}
$$

Taking the sum for $i=0, \ldots, I_{j}-1$ yields the estimate $(4.25)$. For $t \in\left(t_{j, i}, t_{j, i+1}\right)$ we can write

$$
\begin{aligned}
\left\|w_{j}(t)-w(t)\right\|_{L^{2}}^{2} & =\left\|\int_{t_{j, i}}^{t} \dot{w}_{j}(s)-\dot{w}(s) d s\right\|_{L^{2}}^{2} \\
& \leq\left(\int_{t_{j, i}}^{t_{j, i+1}}\left\|\dot{w}_{j}(s)\right\|_{L^{2}}+\|\dot{w}(s)\|_{L^{2}} d s\right)^{2} \\
& \leq 2\left(t_{j, i+1}-t_{j, i}\right) \int_{t_{j, i}}^{t_{j, i+1}}\left\|\dot{w}_{j}(s)\right\|_{L^{2}}^{2}+\|\dot{w}(s)\|_{L^{2}}^{2} d s \\
& \leq 4 \tau_{j} \int_{t_{j, i}}^{t_{j, i+1}}\|\dot{w}(s)\|_{L^{2}}^{2} d s,
\end{aligned}
$$

where in the last inequality we used (4.27). Hence

$$
\int_{t_{j, i}}^{t_{j, i+1}}\left\|w_{j}(t)-w(t)\right\|_{L^{2}}^{2} d t \leq 4 \tau_{j}^{2} \int_{t_{j, i}}^{t_{j, i+1}}\|\dot{w}(t)\|_{L^{2}}^{2} d t .
$$

Taking the sum for $i=0, \ldots, I_{j}-1$ yields the estimate (4.26). 
From $(4.25),(4.26)$ it is clear that $w_{j} \rightarrow w$ in $H^{1}\left(0, T ; L^{2}\right)$ for $\tau_{j} \rightarrow 0$. Let us see that $\left\|\dot{w}_{j}\right\|_{L^{2}} \rightarrow\|\dot{w}\|_{L^{2}}$ in $L^{2}(0, T)$. First, we show that $\left\|\dot{w}_{j}\right\|_{L^{2}} \rightarrow\|\dot{w}\|_{L^{2}}$ a.e. in $(0, T)$. For $t \in\left(t_{j, i}, t_{j, i+1}\right)$

$$
\begin{aligned}
\left|\|\dot{w}(t)\|_{L^{2}}-\left\|\dot{w}_{j}(t)\right\|_{L^{2}}\right| & \leq\left\|\dot{w}(t)-\dot{w}_{j}(t)\right\|_{L^{2}} \leq\left\|\dot{w}(t)-f_{t_{j, i}}^{t_{j, i+1}} \dot{w}(s) d s\right\|_{L^{2}} \\
& \leq\left\|\int_{t_{j, i}}^{t_{j, i+1}} \dot{w}(t)-\dot{w}(s) d s\right\|_{L^{2}} \\
& \leq \int_{t_{j, i}}^{t_{j, i+1}}\|\dot{w}(t)-\dot{w}(s)\|_{L^{2}} d s \\
& \leq 2 \int_{t-\left|t_{j, i+1}-t_{j, i}\right|}^{t+\left|t_{j, i+1}-t_{j, i}\right|}\|\dot{w}(t)-\dot{w}(s)\|_{L^{2}} d s .
\end{aligned}
$$

It is well known (see e.g. [16, Proposition 2.1.22]) that as $\left|t_{j, i+1}-t_{j, i}\right| \rightarrow 0$ the last term is infinitesimal for a.e. $t \in(0, T)$.

Since $\left\|\dot{w}_{j}\right\|_{L^{2}} \rightarrow\|\dot{w}\|_{L^{2}}$ a.e. in $(0, T)$ and $\left\|\dot{w}_{j}\right\|_{L^{2}}$ is bounded in $L^{2}(0, T)$ we know, by classical results, that $\left\|\dot{w}_{j}\right\|_{L^{2}} \rightarrow\|\dot{w}\|_{L^{2}}$ strongly in $L^{1}(0, T)$ and weakly in $L^{2}(0, T)$.

Lemma 4.13. Let $u \in H^{1}\left(0, T ; L^{2}\right)$ be a monotone evolution and assume that $|\partial \mathcal{F}|_{L_{+}^{2}}(\cdot, u(\cdot)) \in L^{2}(0, T)$. Then for every $0 \leq t^{*} \leq T$

$$
\begin{aligned}
\mathcal{E}\left(u\left(t^{*}\right)\right) & \geq \mathcal{E}\left(u_{0}\right)-\int_{0}^{t^{*}}|\partial \mathcal{F}|_{L_{+}^{2}}(t, u(t))\|\dot{u}(t)\|_{L^{2}} d t+\int_{0}^{t^{*}}\langle f(t), \dot{u}(t)\rangle d t \\
& \geq \mathcal{E}\left(u_{0}\right)-\frac{1}{2} \int_{0}^{t^{*}}|\partial \mathcal{F}|_{L_{+}^{2}}^{2}(t, u(t))+\|\dot{u}(t)\|_{L^{2}}^{2} d t+\int_{0}^{t^{*}}\langle f(t), \dot{u}(t)\rangle d t
\end{aligned}
$$

Proof. The lack of time regularity in $H_{0}^{1}$ prevents from employing the chain rule, we will use instead a Riemann sum argument, adapted from [12, Lemma 4.12], see also [22, Proposition 3.8]. Note that $|\partial \mathcal{F}|_{L_{+}^{2}}(\cdot, u(\cdot))<+\infty$ a.e. in $(0, T)$.

Given $t^{*}$ let $0<t_{*}<t^{*}$ with $|\partial \mathcal{F}|_{L_{+}^{2}}\left(t_{*}, u\left(t_{*}\right)\right)<+\infty$. We can find a sequence of finite subdivisions $\mathcal{T}_{j}=\left\{t_{j, i}\right\}$ of $\left[t_{*}, t^{*}\right]$ with $t_{*}=t_{j, 0}<\ldots<t_{j, i}<$ $t_{j, i+1}<\ldots<t_{j, I_{j}}=t^{*}$, such that $\lim _{j \rightarrow+\infty} \max _{i}\left\{t_{j, i+1}-t_{j, i}\right\}=0$ and

$$
S_{j}(\cdot)=\sum_{i=0}^{I_{j}-1}|\partial \mathcal{F}|_{L_{+}^{2}}\left(t_{j, i}, u\left(t_{j, i}\right)\right) \chi_{\left(t_{j, i}, t_{j, i+1}\right)}(\cdot) \rightarrow|\partial \mathcal{F}|_{L_{+}^{2}}(\cdot, u(\cdot))
$$

strongly in $L^{2}\left(t_{*}, t^{*}\right)$, and

$$
F_{j}(\cdot)=\sum_{i=0}^{I_{j}-1} f\left(t_{j, i}\right) \chi_{\left(t_{j, i}, t_{j, i+1}\right)}(\cdot) \rightarrow f \quad \text { strongly in } L^{2}\left(t_{*}, t^{*} ; L^{2}\right) .
$$

(It is enough to apply the Riemann sum argument to the couple $\left(|\partial \mathcal{F}|_{L_{+}^{2}}, f\right) \in$ $L^{2}\left(0, T ; \mathbb{R} \otimes L^{2}\right)$; for sake of clarity, we also remark that, in general, the points 
$\left\{t_{j, i}\right\}$ do not coincide with the points $t_{n, k}=k \tau_{n}$ appearing in the discrete scheme). By convexity of $\mathcal{F}\left(t_{j, i+1}, \cdot\right)$ we write

$$
\begin{aligned}
\mathcal{F}\left(t_{j, i}, u\left(t_{j, i+1}\right)\right) & \geq \mathcal{F}\left(t_{j, i}, u\left(t_{j, i}\right)\right)+d \mathcal{F}\left(t_{j, i}, u\left(t_{j, i}\right)\right)\left[u\left(t_{j, i+1}\right)-u\left(t_{j, i}\right)\right] \\
& \geq \mathcal{F}\left(t_{j, i}, u\left(t_{j, i}\right)\right)-|\partial \mathcal{F}|_{L_{+}^{2}}\left(t_{j, i}, u\left(t_{j, i}\right)\right)\left\|u\left(t_{j, i+1}\right)-u\left(t_{j, i}\right)\right\|_{L^{2}} .
\end{aligned}
$$

Denote by $u_{j}$ the piecewise affine interpolation of $u\left(t_{j, i}\right)$. Writing explicitly the energies $\mathcal{F}\left(t_{j, i}, u\left(t_{j, i+1}\right)\right)$ and $\mathcal{F}\left(t_{j, i}, u\left(t_{j, i}\right)\right)$ we get

$$
\begin{aligned}
\mathcal{E}\left(u\left(t_{j, i+1}\right)\right) \geq & \mathcal{E}\left(u\left(t_{j, i}\right)\right)-\int_{t_{j, i}}^{t_{j, i+1}}|\partial \mathcal{F}|_{L_{+}^{2}}\left(t_{j, i}, u\left(t_{j, i}\right)\right)\left\|\dot{u}_{j}(t)\right\|_{L^{2}} d t \\
& +\int_{t_{j, i}}^{t_{j, i+1}}\left\langle f\left(t_{j, i}\right), \dot{u}_{j}(t)\right\rangle d t .
\end{aligned}
$$

Using the above estimate for $i=1, \ldots, I_{j}$ we get, in terms of the functions $S_{j}$ and $F_{j}$ appearing in (4.28) and (4.29),

$$
\mathcal{E}\left(u\left(t^{*}\right)\right) \geq \mathcal{E}\left(u\left(t_{*}\right)\right)-\int_{t_{*}}^{t^{*}} S_{j}(t)\left\|\dot{u}_{j}(t)\right\|_{L^{2}} d t+\int_{t_{*}}^{t^{*}}\left\langle F_{j}(t), \dot{u}_{j}(t)\right\rangle d t
$$

By (4.28) we known that $S_{j}(\cdot) \rightarrow|\partial \mathcal{F}|_{L_{+}^{2}}(\cdot, u(\cdot))$ strongly in $L^{2}\left(t_{*}, t^{*}\right)$ and by (4.29) that $F_{j} \rightarrow f$ strongly in $L^{2}\left(t_{*}, t^{*} ; L^{2}\right)$. By Lemma 4.12 we get $\dot{u}_{j} \rightarrow \dot{u}$ in $L^{2}\left(t_{*}, t^{*} ; L^{2}\right)$ and $\left\|\dot{u}_{j}\right\|_{L^{2}} \rightarrow\|\dot{u}\|_{L^{2}}$ in $L^{2}\left(t_{*}, t^{*}\right)$. In summary, we can pass to the limit in (4.30) and get, by Young's inequality,

$$
\begin{aligned}
\mathcal{E}\left(u\left(t^{*}\right)\right) \geq & \mathcal{E}\left(u\left(t_{*}\right)\right)-\int_{t_{*}}^{t^{*}}|\partial \mathcal{F}|_{L_{+}^{2}}(t, u(t))\|\dot{u}(t)\|_{L^{2}} d t+\int_{t_{*}}^{t^{*}}\langle f(t), \dot{u}(t)\rangle d t \\
\geq & \mathcal{E}\left(u\left(t_{*}\right)\right)-\frac{1}{2} \int_{t_{*}}^{t^{*}}|\partial \mathcal{F}|_{L_{+}^{2}}^{2}(t, u(t))+\|\dot{u}(t)\|_{L^{2}}^{2} d t \\
& +\int_{t_{*}}^{t^{*}}\langle f(t), \dot{u}(t)\rangle d t .
\end{aligned}
$$

Taking the liminf of the right hand for $t_{*} \rightarrow 0^{+}$we get

$$
\mathcal{E}\left(u\left(t^{*}\right)\right) \geq \mathcal{E}\left(u_{0}\right)-\frac{1}{2} \int_{0}^{t^{*}}|\partial \mathcal{F}|_{L_{+}^{2}}^{2}(t, u(t))+\|\dot{u}(t)\|_{L^{2}}^{2} d t+\int_{0}^{t^{*}}\langle f(t), \dot{u}(t)\rangle d t,
$$

which concludes the proof.

Proof of Theorem 2.3. Clearly, using (2.5) and Lemma 4.13 it follows that for every $0 \leq t^{*} \leq T$ we get the energy identity

$$
\begin{aligned}
\mathcal{E}\left(u\left(t^{*}\right)\right)= & \mathcal{E}\left(u_{0}\right)-\frac{1}{2} \int_{0}^{t^{*}}|\dot{u}(t)|_{L_{+}^{2}}^{2}+|\partial \mathcal{F}|_{L_{+}^{2}}^{2}(t, u(t)) d t \\
& +\int_{0}^{t^{*}}\langle f(t), \dot{u}(t)\rangle d t .
\end{aligned}
$$

Let us prove uniqueness. Remember that in our weak setting solutions belong only to $H^{1}\left(0, T ; L^{2}\right) \cap L^{\infty}\left(0, T ; H_{0}^{1}\right)$, therefore we are not in a position to employ any argument based on the chain rule for $\mathcal{F}$. Instead, we follow the contradiction argument of [19, Theorem 15]. Assume that $u_{\mathrm{I}}$ and $u_{\mathrm{II}}$ are 
different unilateral gradient flows with the same initial value $u_{0}$. Let $t^{*}$ such that $u_{\mathrm{I}}\left(t^{*}\right) \neq u_{\mathrm{II}}\left(t^{*}\right)$. Define $u_{\natural}=\frac{1}{2}\left(u_{\mathrm{I}}+u_{\mathrm{II}}\right)$. Writing the energy identity (4.31) for both $u_{\mathrm{I}}$ and $u_{\mathrm{II}}$ we get (for $i=\mathrm{I}, \mathrm{II}$ )

$$
\begin{aligned}
\frac{1}{2} \mathcal{E}\left(u_{0}\right)= & \frac{1}{2} \mathcal{E}\left(u_{i}\left(t^{*}\right)\right)+\frac{1}{2} \int_{0}^{t^{*}} \frac{1}{2}|\partial \mathcal{F}|_{L_{+}^{2}}^{2}\left(t, u_{i}(t)\right)+\frac{1}{2}\left\|\dot{u}_{i}(t)\right\|_{L^{2}}^{2} d t \\
& -\frac{1}{2} \int_{0}^{t^{*}}\left\langle f(t), \dot{u}_{i}(t)\right\rangle d t .
\end{aligned}
$$

Taking the sum for $i=\mathrm{I}$, II and using the strict convexity of the energy $\mathcal{E}$, the convexity of $\frac{1}{2}\|\cdot\|_{L^{2}}^{2}$, the convexity of $\frac{1}{2}|\partial \mathcal{F}|_{L_{+}^{2}}^{2}(t, \cdot)$ (see Corollary 3.2 ) and the linearity of $\langle f(t), \cdot\rangle$ we get

$\mathcal{E}\left(u_{0}\right)>\mathcal{E}\left(u_{\natural}\left(t^{*}\right)\right)+\frac{1}{2} \int_{0}^{t^{*}}|\partial \mathcal{F}|_{L_{+}^{2}}^{2}\left(t, u_{\natural}(t)\right)+\left\|\dot{u}_{\natural}(t)\right\|_{L^{2}}^{2} d t-\int_{0}^{t^{*}}\left\langle f(t), \dot{u}_{\natural}(t)\right\rangle d t$.

Hence $|\partial \mathcal{F}|_{L_{+}^{2}}\left(\cdot, u_{\natural}(\cdot)\right)$ belongs to $L^{2}\left(0, t^{*}\right)$ and clearly $u_{\natural} \in H^{1}\left(0, T ; L^{2}\right)$. The previous inequality is a contradiction with Lemma 4.13 .

Since the limit evolution is unique it is not necessary to extract any subsequence in Propositions 4.1, 4.5 and 4.9.

Corollary 4.14. Let $u_{n}$ be one the sequences provided by the discrete schemes of Sects. 4.1, 4.2, or 4.3. Then $u_{n} \rightarrow u$ in $H_{0}^{1}$ pointwise in $[0, T]$.

Proof. Given $t^{*} \in[0, T]$ let us first prove that $u_{n}^{\sharp}\left(t^{*}\right) \rightarrow u\left(t^{*}\right)$ in $H_{0}^{1}$. Since $u_{n}^{\sharp}\left(t^{*}\right) \rightarrow u\left(t^{*}\right)$ in $H_{0}^{1}$ it is enough to show that $\mathcal{E}\left(u_{n}^{\sharp}\left(t^{*}\right)\right) \rightarrow \mathcal{E}\left(u\left(t^{*}\right)\right)$, which implies that $u_{n}^{\sharp}\left(t^{*}\right) \rightarrow u\left(t^{*}\right)$ in $H_{0}^{1}$. Let $k_{n}$ such that $t_{n, k_{n}}<t^{*} \leq t_{n, k_{n}+1}$ and recall (4.8), i.e.,

$$
\begin{aligned}
\mathcal{E}\left(u_{n}^{\sharp}\left(t^{*}\right)\right)=\mathcal{E}\left(u_{n}\left(t_{n, k_{n}+1}\right)\right) \leq & \mathcal{E}\left(u_{0}\right)-\int_{0}^{t_{n, k_{n}+1}} \frac{1}{2}\left|\dot{u}_{n}(t)\right|_{L_{+}^{2}}^{2} \\
& +\frac{1}{2}\left|\partial \mathcal{F}_{n}\right|_{L_{+}^{2}}^{2}\left(t, u_{n}^{\sharp}(t)\right)+\left\langle f_{n}(t), \dot{u}_{n}(t)\right\rangle d t .
\end{aligned}
$$

Then,

$$
\begin{aligned}
\mathcal{E}\left(u\left(t^{*}\right)\right) \leq & \liminf _{n \rightarrow+\infty} \mathcal{E}\left(u_{n}^{\sharp}\left(t^{*}\right)\right) \leq \limsup _{n \rightarrow+\infty} \mathcal{E}\left(u_{n}^{\sharp}\left(t^{*}\right)\right) \\
\leq & \mathcal{E}\left(u_{0}\right)-\liminf _{n \rightarrow+\infty} \frac{1}{2} \int_{0}^{t_{n, k_{n}+1}}\left|\dot{u}_{n}(t)\right|_{L_{+}^{2}}^{2}+\left|\partial \mathcal{F}_{n}\right|_{L_{+}^{2}}^{2}\left(t, u_{n}^{\sharp}(t)\right) d t \\
& +\lim _{n \rightarrow+\infty} \int_{0}^{t_{n, k_{n}+1}}\left\langle f_{n}(t), \dot{u}_{n}(t)\right\rangle d t \\
\leq & \mathcal{E}\left(u_{0}\right)-\frac{1}{2} \int_{0}^{t^{*}}|\dot{u}(t)|_{L_{+}^{2}}^{2}+|\partial \mathcal{F}|_{L_{+}^{2}}^{2}(t, u(t)) d t-\int_{0}^{t^{*}}\langle f(t), \dot{u}(t)\rangle d t \\
= & \mathcal{E}\left(u\left(t^{*}\right)\right),
\end{aligned}
$$

where, in the last line, we used (4.9) and (4.11) from Proposition 4.1 together with the energy identity (4.31). As a consequence, all inequalities above turn into equalities and $u_{n}^{\sharp}\left(t^{*}\right) \rightarrow u\left(t^{*}\right)$ in $H_{0}^{1}$; hence $u_{n}\left(t_{n, k_{n}+1}\right)=u_{n}^{\sharp}\left(t^{*}\right) \rightarrow u\left(t^{*}\right)$ in $H_{0}^{1}$. A similar argument shows that $u_{n}\left(t_{n, k_{n}}\right) \rightarrow u\left(t^{*}\right)$ in $H_{0}^{1}$. Being $u_{n}\left(t^{*}\right)$ a 
convex combination of $u_{n}\left(t_{n, k_{n}}\right)$ and $u_{n}\left(t_{n, k_{n}+1}\right)$ it converges strongly to $u\left(t^{*}\right)$ as well.

\section{Further properties of solutions}

\subsection{Parabolic equation}

Proof of Theorem 2.4. When $A u(t)+f(t) \in L^{2}$ it is defined the $L^{2}$-gradient of the energy $\mathcal{E}$ in $u(t)$, i.e.,

$$
d \mathcal{E}(u(t))[v]=-(A u(t), v)=\langle\nabla \mathcal{E}(u(t)), v\rangle .
$$

In a similar way, $\nabla \mathcal{F}(t, u(t))=-A u(t)-f(t)$, in particular

$$
|\partial \mathcal{F}|_{L_{+}^{2}}(t, u(t))=\left\|[A u(t)+f(t)]_{+}\right\|_{L^{2}}=\left\|[-\nabla \mathcal{F}(t, u(t))]_{+}\right\|_{L^{2}} .
$$

Under the regularity assumptions of Theorem 2.4 we know, e.g. by [10, Lemma 3.3], that the energy $t \mapsto \mathcal{E}(u(t))$ is absolutely continuous and that a.e. in $(0, T)$ it holds

$$
\dot{\mathcal{E}}(u(t))=\langle\nabla \mathcal{E}(u(t)), \dot{u}(t)\rangle=\langle\nabla \mathcal{F}(t, u(t)), \dot{u}(t)\rangle+\langle f(t), \dot{u}(t)\rangle
$$

Assume that $u$ is a solution of $(2.7)$, i.e. $\dot{u}(t)=[-\nabla \mathcal{F}(t, u(t))]_{+}$, then

$$
\begin{aligned}
\langle\nabla \mathcal{E}(u(t)), \dot{u}(t)\rangle= & -\langle-\nabla \mathcal{F}(t, u(t)), \dot{u}(t)\rangle+\langle f(t), \dot{u}(t)\rangle \\
= & -\left\langle[-\nabla \mathcal{F}(t, u(t))]_{+}-[-\nabla \mathcal{F}(t, u(t))]_{-},[-\nabla \mathcal{F}(t, u(t))]_{+}\right\rangle \\
& +\langle f(t), \dot{u}(t)\rangle \\
= & -\left\|[-\nabla \mathcal{F}(t, u(t))]_{+}\right\|_{L^{2}}^{2}+\langle f(t), \dot{u}(t)\rangle \\
= & -\frac{1}{2}|\dot{u}(t)|_{L_{+}^{2}}^{2}-\frac{1}{2}|\partial \mathcal{F}|_{L_{+}^{2}}^{2}(t, u(t))+\langle f(t), \dot{u}(t)\rangle
\end{aligned}
$$

Taking the integral in time gives Definition 2.2.

Conversely, assume that $u$ is the unilateral gradient flow given by Theorem 2.3. Being $\dot{u} \geq 0$ we get

$$
\begin{aligned}
\dot{\mathcal{E}}(u(t)) & =\langle\nabla \mathcal{F}(t, u(t)), \dot{u}(t)\rangle+\langle f(t), \dot{u}(t)\rangle \\
& =-\left\langle[-\nabla \mathcal{F}(t, u(t))]_{+}-[-\nabla \mathcal{F}(t, u(t))]_{-}, \dot{u}(t)\right\rangle+\langle f(t), \dot{u}(t)\rangle \\
& \geq-\left\langle[-\nabla \mathcal{F}(t, u(t))]_{+}, \dot{u}(t)\right\rangle+\langle f(t), \dot{u}(t)\rangle \\
& \geq-\left\|[-\nabla \mathcal{F}(t, u(t))]_{+}\right\|_{L^{2}}\|\dot{u}(t)\|_{L^{2}}+\langle f(t), \dot{u}(t)\rangle \\
& \geq-\frac{1}{2}\|\dot{u}(t)\|_{L^{2}}^{2}-\frac{1}{2}\left\|[-\nabla \mathcal{F}(t, u(t))]_{+}\right\|_{L^{2}}^{2}+\langle f(t), \dot{u}(t)\rangle
\end{aligned}
$$

Since $\dot{\mathcal{E}}(u(t))=-\frac{1}{2}\|\dot{u}(t)\|_{L^{2}}^{2}-\frac{1}{2}\|\nabla \mathcal{F}(t, u(t))\|_{L^{2}}^{2}+\langle f(t), \dot{u}(t)\rangle$ all the above inequalities turn into equalities. In particular,

$$
\begin{aligned}
\left\langle[-\nabla \mathcal{F}(t, u(t))]_{+}, \dot{u}(t)\right\rangle & =\left\|[-\nabla \mathcal{F}(t, u(t))]_{+}\right\|_{L^{2}}\|\dot{u}(t)\|_{L^{2}} \\
& =\frac{1}{2}\|\dot{u}(t)\|_{L^{2}}^{2}+\frac{1}{2}\left\|[-\nabla \mathcal{F}(t, u(t))]_{+}\right\|_{L^{2}}^{2}
\end{aligned}
$$

then Cauchy inequality implies $\dot{u}(t)=[-\nabla \mathcal{F}(t, u(t))]_{+}=[A u(t)+f(t)]_{+}$. 
Proof of Theorem 2.5. Let $u$ be the unilateral gradient flow for $\mathcal{F}$ with initial datum $u_{0}$, in the sense of Definition 2.2. By the energy identity we know that $\|\dot{u}(t)\|_{L^{2}}=|\partial \mathcal{F}|_{L_{+}^{2}}(t, u(t))$ is a.e. finite in $[0, T]$. Hence, by Corollary 3.8, for a.e. $t \in[0, T]$ we have

$$
\|\dot{u}(t)\|_{L^{2}}=|\partial \mathcal{F}|_{L_{+}^{2}}(t, u(t))=\left\|[A u(t)+f(t)]_{+}\right\|_{L^{2}} .
$$

Now, let us show that $-d \mathcal{F}(t, u(t)) \leq \dot{u}(t)$ in $H^{-1}$ for a.e. $t$ in $[0, T]$. By uniqueness, we can rely on the discrete scheme of Sect.4.1. By (4.4) for every $t \in\left(t_{n, k}, t_{n, k+1}\right)$ we have

$$
-d \mathcal{F}_{n}\left(t, u_{n}^{\sharp}(t)\right)[\phi] \leq\left\langle\dot{u}_{n}(t), \phi\right\rangle \quad \text { for every } \phi \in C_{0}^{\infty} \text { with } \phi \geq 0,
$$

which reads, by symmetry of $a(\cdot, \cdot)$,

$$
\left\langle u_{n}^{\sharp}(t), A \phi\right\rangle+\left\langle f_{n}(t), \phi\right\rangle \leq\left\langle\dot{u}_{n}(t), \phi\right\rangle .
$$

Thus, for every $0 \leq t_{1}<t_{2} \leq T$ we can write

$$
\int_{t_{1}}^{t_{2}}\left\langle u_{n}^{\sharp}(t), A \phi\right\rangle+\left\langle f_{n}(t), \phi\right\rangle d t \leq \int_{t_{1}}^{t_{2}}\left\langle\dot{u}_{n}(t), \phi\right\rangle d t .
$$

Passing to the limit, by the strong convergence of $u_{n}^{\sharp}(t)$ in $L^{2}\left(0, T ; L^{2}\right)$ together with the strong convergence of $f_{n}$ in $L^{2}\left(0, T ; L^{2}\right)$ and the weak convergence of $u_{n}$ in $H^{1}\left(0, T ; L^{2}\right)$, we get

$$
\int_{t_{1}}^{t_{2}}\langle u(t), A \phi\rangle+\langle f(t), \phi\rangle d t \leq \int_{t_{1}}^{t_{2}}\langle\dot{u}(t), \phi\rangle d t .
$$

Since the above inequality holds for any choice of $t_{1}<t_{2}$, for a.e. $t \in(0, T)$ we have

$$
-d \mathcal{F}(t, u(t))[\phi]=\langle u(t), A \phi\rangle+\langle f(t), \phi\rangle \leq\langle\dot{u}(t), \phi\rangle,
$$

which reads $(A u(t)+f(t), \phi) \leq\langle\dot{u}(t), \phi\rangle$. Therefore, applying Lemma 3.7 we get $[A u(t)+f(t)]_{+} \leq \dot{u}(t)$. By $(5.2)$, we have $\|\dot{u}(t)\|_{L^{2}}=\left\|[A u(t)+f(t)]_{+}\right\|_{L^{2}}$, it follows that $\dot{u}(t)=[A u(t)+f(t)]_{+}$.

\subsection{Non-uniqueness for the parabolic PDE in $L^{2}$}

We provide an example in which the parabolic problem (2.8) has many solutions, and thus it is not equivalent to Definition 2.2. Let $u_{0} \in H_{0}^{1}(-1,1)$ be defined by $u_{0}(x)=1-|x|$. We will denote by $u^{\prime}$ and $u^{\prime \prime}$ the first and second space derivatives, respectively. Clearly $u_{0}^{\prime \prime}=-2 \delta_{0}$, where $\delta_{0}$ denotes the Dirac delta in the origin. Note that $u_{0}>0$ in $(-1,1)$ and $\left[u_{0}^{\prime \prime}\right]_{+}=0$.

Let $f=u_{0}$ and consider the energy $\mathcal{F}: H_{0}^{1}(-1,1) \rightarrow \mathbb{R}$ given by

$$
\mathcal{F}(u)=\frac{1}{2} \int_{(-1,1)}\left|u^{\prime}\right|^{2} d x-\int_{(-1,1)} f u d x=\mathcal{E}(u)-\langle f, u\rangle .
$$

Clearly $A u+f=u^{\prime \prime}+f$. Let us define $u(t)=(1+t) u_{0}$. Then, $\dot{u}(t)=u_{0}$ and $u^{\prime \prime}(t)=(1+t) u_{0}^{\prime \prime}=-2(1+t) \delta_{0}$. Since $\left[u^{\prime \prime}(t)+f\right]_{+}=\left[-2(1+t) \delta_{0}+u_{0}\right]_{+}=u_{0}$, it turns out that $u$ solves

$$
\left\{\begin{array}{l}
\dot{u}(t)=\left[u^{\prime \prime}(t)+f\right]_{+} \quad \text { in }(0, T) \\
u(0)=u_{0}
\end{array}\right.
$$


On the other hand $u$ does not satisfy the energy identity

$$
\mathcal{E}(u(t))=\mathcal{E}\left(u_{0}\right)-\int_{0}^{t}\|\dot{u}(t)\|_{L^{2}}^{2} d t+\int_{0}^{t}\langle f, \dot{u}(t)\rangle d t .
$$

which is equivalent to (2.5). Indeed, $\mathcal{E}(u(t))=\frac{1}{2}(1+t)^{2} \int_{(-1,1)}\left|u_{0}^{\prime}\right|^{2} d x=$ $(1+t)^{2}, \mathcal{E}\left(u_{0}\right)=1,\|\dot{u}(t)\|_{L^{2}}^{2}=\left\|u_{0}\right\|_{L^{2}}^{2}$ while $\langle f, \dot{u}(t)\rangle=\left\|u_{0}\right\|_{L^{2}}^{2}$.

Solution of the unilateral gradient flow. The solution of the unilateral gradient flow, in the sense of Definition 2.2, is computed hereafter. Let us start considering the sub-interval $(0,1)$ and the following parabolic problem

$$
\begin{cases}\dot{u}(t, x)=u^{\prime \prime}(t, x)+f(x) & \text { in }(0, T) \times(0,1) \\ u(t, 0)=1, u(t, 1)=0 & \text { for } t \in(0, T) \\ u(0, x)=u_{0} & \text { for } x \in(0,1) .\end{cases}
$$

By classical results, see e.g. [15, Theorem 5 (ii) $\S 7.1]$ there exists a unique solution $u_{r}$ which belongs to $L^{\infty}\left(0, T ; H^{2}(0,1)\right) \cap H^{1}\left(0, T ; H^{1}(0,1)\right)$.

Lemma 5.1. Let $u_{r}$ be the solution of the above parabolic problem. Then $\dot{u}_{r}(t) \geq$ 0 , and thus

$$
\begin{cases}\dot{u}_{r}(t, x)=\left[u_{r}^{\prime \prime}(t, x)+f(x)\right]_{+} & \text {in }(0, T) \times(0,1) \\ u_{r}(t, 0)=1, u_{r}(t, 1)=0 & \text { for } t \in(0, T) \\ u_{r}(0, x)=u_{0} & \text { for } x \in(0,1) .\end{cases}
$$

Moreover $u_{r}^{\prime}(t, 0)<0$ for a.e. $t \in(0, T)$.

Proof. First, let us prove that $\dot{u}_{r} \geq 0$. For convenience, we introduce the set $\mathcal{U}_{r}=\left\{u \in H^{1}(0,1): u(0)=1, u(1)=0\right\}$ and the energy $\mathcal{F}_{r}: \mathcal{U}_{r} \rightarrow \mathbb{R}$ given by

$$
\mathcal{F}_{r}(u)=\frac{1}{2} \int_{(0,1)}\left|u^{\prime}\right|^{2} d x-\int_{(0,1)} f u d x .
$$

Consider again an implicit (unconstrained) Euler scheme. Let $\tau>0$ such that $T / \tau$ is integer and let $t_{k}=k \tau$ for $k=0, \ldots, T / \tau$. Given $u_{0}$ we define by induction

$$
u_{k+1} \in \operatorname{argmin}\left\{\mathcal{F}_{r}(u)+\frac{1}{2 \tau}\left\|u-u_{k}\right\|_{L^{2}}^{2}: u \in \mathcal{U}_{r}\right\} .
$$

We will prove by induction that $u_{k+1} \geq u_{k}$ for every index $k$. Let us see that $u_{1} \geq u_{0}$. Clearly, being $u_{0}$ affine,

$$
\int_{(0,1)} u_{0}^{\prime} \phi^{\prime} d x=0, \quad \text { for every } \phi \in H_{0}^{1}(0,1) .
$$

Consider the auxiliary function $u_{*}=u_{0}+\left|u_{1}-u_{0}\right|$ and note that

$$
u_{*} \geq \max \left\{u_{0}, u_{1}\right\}, \quad\left|u_{*}-u_{0}\right|=\left|u_{1}-u_{0}\right|, \quad|| u_{1}-\left.u_{0}\right|^{\prime}|=|\left(u_{1}-u_{0}\right)^{\prime} \mid .
$$

We claim that $\mathcal{F}_{r}\left(u_{*}\right) \leq \mathcal{F}_{r}\left(u_{1}\right)$. Indeed, since $\left(u_{1}-u_{0}\right)$ and $\left|u_{1}-u_{0}\right|$ belong to $H_{0}^{1}(0,1)$ we can write 


$$
\begin{aligned}
\mathcal{F}_{r}\left(u_{1}\right) & =\mathcal{F}_{r}\left(u_{0}+\left(u_{1}-u_{0}\right)\right) \\
& =\int_{(0, L)} \frac{1}{2}\left|u_{0}^{\prime}\right|^{2}+\frac{1}{2}\left|\left(u_{1}-u_{0}\right)^{\prime}\right|^{2}+u_{0}^{\prime}\left(u_{1}-u_{0}\right)^{\prime}-f u_{1} d x \\
& =\int_{(0, L)} \frac{1}{2}\left|u_{0}^{\prime}\right|^{2}+\frac{1}{2}\left|\left(u_{1}-u_{0}\right)^{\prime}\right|^{2}-f u_{1} d x
\end{aligned}
$$

and

$$
\begin{aligned}
\mathcal{F}_{r}\left(u_{*}\right) & =\mathcal{F}_{r}\left(u_{0}+\left|u_{1}-u_{0}\right|\right) \\
& =\int_{(0, L)} \frac{1}{2}\left|u_{0}^{\prime}\right|^{2}+\frac{1}{2}|| u_{1}-\left.\left.u_{0}\right|^{\prime}\right|^{2}+u_{0}^{\prime}\left|u_{1}-u_{0}\right|^{\prime}-f u_{*} d x \\
& =\int_{(0, L)} \frac{1}{2}\left|u_{0}^{\prime}\right|^{2}+\frac{1}{2}\left|\left(u_{1}-u_{0}\right)^{\prime}\right|^{2}-f u_{*} d x .
\end{aligned}
$$

Since $u_{*} \geq u_{1}$ and $f=u_{0}>0$ we have $\mathcal{F}_{r}\left(u_{*}\right) \leq \mathcal{F}_{r}\left(u_{1}\right)$. As $\left|u_{*}-u_{0}\right|=\left|u_{1}-u_{0}\right|$ from the latter inequality it follows that

$$
\mathcal{F}_{r}\left(u_{*}\right)+\frac{1}{2 \tau}\left\|u_{*}-u_{0}\right\|_{L^{2}}^{2} \leq \mathcal{F}_{r}\left(u_{1}\right)+\frac{1}{2 \tau}\left\|u_{1}-u_{0}\right\|_{L^{2}}^{2}
$$

and then, by uniqueness of the minimizer in (5.5), that $u_{1}=u_{*} \geq \max \left\{u_{0}, u_{1}\right\}$, hence $u_{1} \geq u_{0}$.

Next, let us see that $u_{k+1} \geq u_{k}$ for $k \geq 1$. In this case, we employ the Euler-Lagrange equation for $u_{k}$, i.e.,

$$
\int_{(0,1)} u_{k}^{\prime} \phi^{\prime}-f \phi+\frac{1}{\tau}\left(u_{k}-u_{k-1}\right) \phi d x=0 \quad \text { for every } \phi \in H_{0}^{1}(0,1) .
$$

As in the case $k=0$, we check that $\mathcal{F}_{r}\left(u_{*}\right) \leq \mathcal{F}\left(u_{k+1}\right)$ for $u_{*}=u_{k}+\left|u_{k+1}-u_{k}\right|$. Choosing $\phi=u_{k+1}-u_{k}$ and $\phi=\left|u_{k+1}-u_{k}\right|$ in the Euler-Lagrange equation yields, respectively,

$$
\begin{aligned}
\mathcal{F}_{r}\left(u_{k+1}\right)= & \mathcal{F}_{r}\left(u_{k}+\left(u_{k+1}-u_{k}\right)\right) \\
= & \int_{(0,1)} \frac{1}{2}\left|u_{k}^{\prime}\right|^{2}+\frac{1}{2}\left|\left(u_{k+1}-u_{k}\right)^{\prime}\right|^{2}+u_{k}^{\prime}\left(u_{k+1}-u_{k}\right)^{\prime} d x \\
& -\int_{(0,1)} f u_{k}+f\left(u_{k+1}-u_{k}\right) d x \\
= & \int_{(0,1)} \frac{1}{2}\left|u_{k}^{\prime}\right|^{2}+\frac{1}{2}\left|\left(u_{k+1}-u_{k}\right)^{\prime}\right|^{2}-f u_{k} d x \\
& -\frac{1}{\tau} \int_{(0,1)}\left(u_{k}-u_{k-1}\right)\left(u_{k+1}-u_{k}\right) d x
\end{aligned}
$$

and

$$
\begin{aligned}
\mathcal{F}_{r}\left(u_{*}\right)= & \mathcal{F}_{r}\left(u_{k}+\left|u_{k+1}-u_{k}\right|\right) \\
= & \int_{(0,1)} \frac{1}{2}\left|u_{k}^{\prime}\right|^{2}+\frac{1}{2}|| u_{k+1}-\left.\left.u_{k}\right|^{\prime}\right|^{2}+u_{k}^{\prime}\left|u_{k+1}-u_{k}\right|^{\prime} d x \\
& -\int_{(0,1)} f u_{k}+f\left|u_{k+1}-u_{k}\right| d x
\end{aligned}
$$




$$
\begin{aligned}
= & \int_{(0,1)} \frac{1}{2}\left|u_{k}^{\prime}\right|^{2}+\frac{1}{2}\left|\left(u_{k+1}-u_{k}\right)^{\prime}\right|^{2}-f u_{k} d x \\
& -\frac{1}{\tau} \int_{(0,1)}\left(u_{k}-u_{k-1}\right)\left|u_{k+1}-u_{k}\right| d x .
\end{aligned}
$$

By induction $u_{k} \geq u_{k-1}$ and $\left(u_{k}-u_{k-1}\right)\left(u_{k+1}-u_{k}\right) \leq\left(u_{k}-u_{k-1}\right)\left|u_{k+1}-u_{k}\right|$. Hence $\mathcal{F}_{r}\left(u_{*}\right) \leq \mathcal{F}\left(u_{k+1}\right)$.

It is well known that up to subsequences the piecewise affine interpolation $u_{\tau}$ converges weakly in $H^{1}\left(0, T ; L^{2}(0,1)\right)$ to the unique solution $u_{r}$ of $(5.4)$. Therefore, $u_{r}$ is monotone non-decreasing in time.

It is simple to check that the minimizer of the energy $\mathcal{F}_{r}$ in $\mathcal{U}_{r}$ is the function

$$
u_{\min }(x)=-\frac{1}{6}(1-x)^{3}+\frac{7}{6}(1-x) .
$$

Since $u_{0}<u_{\text {min }}$, the comparison principle for (5.4) implies $u_{0} \leq u(t) \leq u_{\text {min }}$ in $(0, T)$. Moreover, $u_{0}(t, 0)=u_{r}(t, 0)=u_{m i n}(t, 0)=1$, hence $u_{r}^{\prime}(t, 0) \leq$ $u_{\min }^{\prime}(t, 0)=-2 / 3$.

Proposition 5.2. The function $u$ defined by

$$
u(t, x)= \begin{cases}u_{r}(t, x) & x \in(0,1) \\ u_{r}(t,-x) & x \in(-1,0)\end{cases}
$$

is the unilateral gradient flow for the functional $\mathcal{F}$ with initial conditions $u_{0}(x)=1-|x|$.

Proof. For convenience, denote $u_{l}(t, x)=u_{r}(t,-x)$ and note that, by previous lemma, it holds $\dot{u}_{l}(t)=\left[u_{l}^{\prime \prime}(t)+f\right]_{+}$.

Moreover, in terms of $u_{l}$ and $u_{r}$ the derivatives of $u$ read

$$
\begin{aligned}
\dot{u}(t) & =\dot{u}_{l}(t) \mathcal{L}_{\mid(-1,0)}+\dot{u}_{r}(t) \mathcal{L}_{\mid(0,1)}, \\
u^{\prime \prime}(t) & =u_{l}^{\prime \prime}(t) \mathcal{L}_{\mid(-1,0)}+u_{r}^{\prime \prime}(t) \mathcal{L}_{\mid(0,1)}+\left[u_{r}^{\prime}(t, 0)-u_{l}^{\prime}(t, 0)\right] \delta_{0},
\end{aligned}
$$

where $\mathcal{L}$ and $\delta_{0}$ denote respectively the Lebesgue measure and the Dirac delta. We remark that $\dot{u}(t, 0)=0$. Note that $\left[u_{r}^{\prime}(t, 0)-u_{l}^{\prime}(t, 0)\right]=2 u_{r}^{\prime}(t, 0)<0$, hence

$$
\left[u^{\prime \prime}(t)+f\right]_{+}=\left[u_{l}^{\prime \prime}(t)+f\right]_{+} \mathcal{L}_{\mid(-1,0)}+\left[u_{r}^{\prime \prime}(t)+f\right]_{+} \mathcal{L}_{\mid(0,1)} .
$$

In particular $\dot{u}(t)=\left[u^{\prime \prime}(t)+f\right]_{+}$.

By the regularity of $f$ we can characterize the unilateral gradient flow for $\mathcal{F}$ as in Proposition 2.12, i.e.

$$
\dot{\mathcal{F}}(u(t)) \leq-\frac{1}{2}|\dot{u}(t)|_{L_{+}^{2}}^{2}-\frac{1}{2}|\partial \mathcal{F}|_{L_{+}^{2}}^{2}(u(t))=-\frac{1}{2}\|\dot{u}(t)\|_{L^{2}}^{2}-\frac{1}{2}\left\|\left[u^{\prime \prime}(t)+f\right]_{+}\right\|_{L^{2}}^{2} .
$$

Being $u \in H^{1}\left(0, T ; H_{0}^{1}(-1,1)\right)$ the chain rule and the fact that $\dot{u}(t, 0)=0$ yield

$$
\begin{aligned}
\dot{\mathcal{F}}(u(t))= & d \mathcal{F}(u(t))[\dot{u}(t)]=-\left(u^{\prime \prime}(t)+f, \dot{u}(t)\right) \\
= & -\left\langle u_{l}^{\prime \prime}(t)+f, \dot{u}_{l}(t)\right\rangle-\left\langle u_{r}^{\prime \prime}(t)+f, \dot{u}_{r}(t)\right\rangle \\
& -\left(\left[u_{r}^{\prime}(t, 0)-u_{l}^{\prime}(t, 0)\right] \delta_{0}, \dot{u}(t, 0)\right) \\
= & -\left\|\dot{u}_{l}(t)\right\|_{L^{2}}^{2}-\left\|\dot{u}_{r}(t)\right\|_{L^{2}}^{2}=-\|\dot{u}(t)\|_{L^{2}}^{2}=-\left\|\left[u^{\prime \prime}(t)+f\right]_{+}\right\|_{L^{2}}^{2},
\end{aligned}
$$


which concludes the proof.

\subsection{Comparison principle}

Proof of Proposition 2.10. Since the unilateral gradient flow is unique it is enough to prove the maximum principle for the discrete solutions provided in Sect. 4.2; indeed, if $u_{n, k} \leq v_{n, k}$ for every $k \geq 0$ then $u_{n} \leq v_{n}$ in $[0, T]$ and passing to the limit weakly in $H^{1}\left(0, T ; L^{2}\right)$ we get $u \leq v$ in $[0, T]$. To this end, fix $\tau_{n}>0$ and assume that $u_{n, 0}=u_{0} \leq v_{0}=v_{n, 0}$. We will show by induction that $u_{n, k} \leq v_{n, k}$ for every index $k \geq 1$. We recall that $u_{n, k+1}=$ $\max \left\{\tilde{u}_{n, k+1}, u_{n, k}\right\}$ and that

$$
\tilde{u}_{n, k+1} \in \operatorname{argmin}\left\{\mathcal{F}\left(t_{n, k+1}, u\right)+\frac{1}{2 \tau_{n}}\left\|u-u_{n, k}\right\|_{L^{2}}^{2}: u \in H_{0}^{1}\right\} .
$$

In a similar way, $v_{n, k+1}=\max \left\{\tilde{v}_{n, k+1}, v_{n, k}\right\}$ and

$$
\tilde{v}_{n, k+1} \in \operatorname{argmin}\left\{\mathcal{F}\left(t_{n, k+1}, v\right)+\langle f-g, v\rangle+\frac{1}{2 \tau_{n}}\left\|v-v_{n, k}\right\|_{L^{2}}^{2}: u \in H_{0}^{1}\right\} .
$$

By minimality there exists $\xi_{n, k+1} \in \partial \mathcal{F}\left(t_{n, k+1}, \tilde{u}_{n, k+1}\right) \subset H^{-1}$ such that $\xi_{n, k+1}+\frac{1}{\tau_{n}}\left(\tilde{u}_{n, k+1}-u_{n, k}\right)=0$ in $H^{-1}$. In a similar way, there exists $\zeta_{n, k+1} \in$ $\partial \mathcal{F}\left(t_{n, k+1}, \tilde{v}_{n, k+1}\right)$ such that $\zeta_{n, k+1}+(f-g)+\frac{1}{\tau_{n}}\left(\tilde{v}_{n, k+1}-v_{n, k}\right)=0$ in $H^{-1}$. Assume by induction that $u_{n, k} \leq v_{n, k}$, we claim that $\tilde{u}_{n, k+1} \leq \tilde{v}_{n, k+1}$ from which we get $u_{n, k+1} \leq v_{n, k+1}$. Using $\left[\tilde{u}_{n, k+1}-\tilde{v}_{n, k+1}\right]_{+} \in H_{0}^{1}$ as a test function we get

$$
\begin{aligned}
& \left(\xi_{n, k+1}-\zeta_{n, k+1},\left[\tilde{u}_{n, k+1}-\tilde{v}_{n, k+1}\right]_{+}\right)+\left\langle g-f,\left[\tilde{u}_{n, k+1}-\tilde{v}_{n, k+1}\right]_{+}\right\rangle \\
& \quad+\frac{1}{\tau_{n}}\left\langle\left(\tilde{u}_{n, k+1}-\tilde{v}_{n, k+1}\right)-\left(u_{n, k}-v_{n, k}\right),\left[\tilde{u}_{n, k+1}-\tilde{v}_{n, k+1}\right]_{+}\right\rangle=0 .
\end{aligned}
$$

The first term is non-negative by $T$-monotonicity, see Remark 3.5, the second is non-negative because $f \leq g$, hence for the last term we can write

$$
\int_{\Omega}\left(u_{n, k}-v_{n, k}\right)\left[\tilde{u}_{n, k+1}-\tilde{v}_{n, k+1}\right]_{+} d x \geq \int_{\Omega}\left[\tilde{u}_{n, k+1}-\tilde{v}_{n, k+1}\right]_{+}^{2} d x \geq 0 .
$$

Since $u_{n, k} \leq v_{n, k}$ the integrand in the left hand side is non-positive; it follows that $\left[\tilde{u}_{n, k+1}-\tilde{v}_{n, k+1}\right]_{+}=0$ and thus $\tilde{u}_{n, k+1} \leq \tilde{v}_{n, k+1}$.

\subsection{Continuous dependence}

Proof of Proposition 2.11. We adopt the scheme of [23]. Denote $\mathcal{F}^{m}(t, u)=$ $\mathcal{E}(u)-\left\langle u, f^{m}(t)\right\rangle$. By definition we know that for every $0 \leq t^{*} \leq T$ it holds

$$
\begin{aligned}
\mathcal{E}\left(u^{m}\left(t^{*}\right)\right) \leq & \mathcal{E}\left(u_{0}^{m}\right)-\frac{1}{2} \int_{0}^{t^{*}}\left\|\dot{u}^{m}(t)\right\|_{L^{2}}^{2}+\left|\partial \mathcal{F}^{m}\right|_{L_{+}^{2}}^{2}\left(t, u^{m}(t)\right) d t \\
& +\int_{0}^{t^{*}}\left\langle f^{m}(t), \dot{u}^{m}(t)\right\rangle d t .
\end{aligned}
$$

We recall that by Theorem 2.5 and Corollary 3.8 we have $\left\|\dot{u}^{m}(t)\right\|_{L^{2}}=\|\left[A u^{m}(t)+\right.$ $\left.f^{m}(t)\right]_{+} \|_{L^{2}}=\left|\partial \mathcal{F}^{m}\right|_{L_{+}^{2}}\left(t, u^{m}(t)\right)$. Hence, choosing $t^{*}=T$ above we obtain

$$
\begin{aligned}
\mathcal{E}\left(u_{0}^{m}\right) & \geq \int_{0}^{T}\left\|\dot{u}^{m}(t)\right\|_{L^{2}}^{2}-\int_{0}^{T}\left\langle f^{m}(t), \dot{u}^{m}(t)\right\rangle d t \\
& \geq\left\|\dot{u}^{m}\right\|_{L^{2}\left(0, T ; L^{2}\right)}^{2}-\left\|f^{m}\right\|_{L^{2}\left(0, T ; L^{2}\right)}\left\|\dot{u}^{m}\right\|_{L^{2}\left(0, T ; L^{2}\right)} .
\end{aligned}
$$


Since $u_{0}^{m} \rightarrow u_{0}$ in $H_{0}^{1}$ we have $\mathcal{E}\left(u_{0}^{m}\right) \rightarrow \mathcal{E}\left(u_{0}\right)$; since $f^{m} \rightarrow f$ in $L^{2}\left(0, T ; L^{2}\right)$ the above estimate implies that the sequence $\dot{u}^{m}$ is bounded in $L^{2}\left(0, T ; L^{2}\right)$. It follows that $u_{m}$ is bounded in $H^{1}\left(0, T ; L^{2}\right)$. Moreover, by $(5.6)$ and by coercivity of the stored energy, for every $0 \leq t^{*} \leq T$ we have

$$
c\left\|u^{m}\left(t^{*}\right)\right\|_{H_{0}^{1}}^{2} \leq \mathcal{E}\left(u^{m}\left(t^{*}\right)\right) \leq \mathcal{E}\left(u_{0}^{m}\right)+\left\|f^{m}\right\|_{L^{2}\left(0, T ; L^{2}\right)}\left\|\dot{u}^{m}\right\|_{L^{2}\left(0, T ; L^{2}\right)} \leq C .
$$

Hence, the sequence $u_{m}$ is bounded also in $L^{\infty}\left(0, T ; H_{0}^{1}\right)$. In conclusion, there exists a subsequence (non relabeled) such that $u^{m} \rightarrow u$ in $H^{1}\left(0, T ; L^{2}\right)$; clearly $u_{m}$ is monotone non-decreasing. Moreover, arguing as in the proof of Proposition 4.1, we get that $u_{m}(t) \rightarrow u(t)$ in $H_{0}^{1}$ for every $t \in[0, T]$.

It remains to show that $u$ is the unilateral gradient flow for $\mathcal{F}$ with initial condition $u_{0}$. By $(5.6)$ for every $t^{*} \in(0, T]$ we can write

$$
\begin{aligned}
\mathcal{E}\left(u\left(t^{*}\right)\right) \leq & \liminf _{m \rightarrow+\infty} \mathcal{E}\left(u^{m}\left(t^{*}\right)\right) \\
\leq & \limsup _{m \rightarrow+\infty} \mathcal{E}\left(u_{0}^{m}\right) \\
& -\frac{1}{2} \liminf _{m \rightarrow+\infty}\left(\int_{0}^{t^{*}}\left\|\dot{u}^{m}(t)\right\|_{L^{2}}^{2}+\left|\partial \mathcal{F}^{m}\right|_{L_{+}^{2}}^{2}\left(t, u^{m}(t)\right) d t\right) \\
& +\limsup _{m \rightarrow+\infty} \int_{0}^{t^{*}}\left\langle f^{m}(t), \dot{u}^{m}(t)\right\rangle d t .
\end{aligned}
$$

We know that $\mathcal{E}\left(u_{0}^{m}\right) \rightarrow \mathcal{E}\left(u_{0}\right)$ because $u_{0}^{m} \rightarrow u_{0}$ (strongly) in $H_{0}^{1}$. Since $\dot{u}^{m} \rightarrow \dot{u}$ in $L^{2}\left(0, T ; L^{2}\right)$ we have $\|\dot{u}\|_{L^{2}\left(0, t^{*} ; L^{2}\right)}^{2} \leq \liminf _{m \rightarrow+\infty}\left\|\dot{u}^{m}\right\|_{L^{2}\left(0, t^{*} ; L^{2}\right)}^{2}$. As $u^{m} \rightarrow u$ in $H_{0}^{1}$ a.e. in $(0, T)$ we can apply Corollary 3.4 and then by Fatou's lemma we get

$$
\int_{0}^{t^{*}}|\partial \mathcal{F}|_{L_{+}^{2}}^{2}(t, u(t)) d t \leq \liminf _{m \rightarrow+\infty} \int_{0}^{t^{*}}\left|\partial \mathcal{F}^{m}\right|_{L_{+}^{2}}^{2}\left(t, u^{m}(t)\right) d t
$$

Finally, $\int_{0}^{t^{*}}\left\langle f^{m}(t), \dot{u}^{m}(t)\right\rangle d t \rightarrow \int_{0}^{t^{*}}\langle f(t), \dot{u}(t)\rangle d t$ by strong-weak convergence in $L^{2}\left(0, t^{*} ; L^{2}\right)$. In conclusion, we get

$\mathcal{E}\left(u\left(t^{*}\right)\right) \leq \mathcal{E}(u(0))-\frac{1}{2} \int_{0}^{t^{*}}|\dot{u}(t)|_{L_{+}^{2}}^{2}+|\partial \mathcal{F}|_{L_{+}^{2}}^{2}(t, u(t)) d t+\int_{0}^{t^{*}}\langle f(t), \dot{u}(t)\rangle d t$,

which is $(2.5)$.

In order to prove that $u_{m}(t) \rightarrow u(t)$ strongly in $H_{0}^{1}$ for every $t \in[0, T]$ it is enough to follow the proof of the strong convergence in Sect. 4.4.

\section{Solutions for $f \in A C\left(0, T ; L^{2}\right)$}

In this section we consider the case in which $f \in A C\left(0, T ; L^{2}\right)$ and we will prove the assertions contained in Proposition 2.12. 


\subsection{Characterization by power balance}

First we prove (2.12). If $f \in A C\left(0, T ; L^{2}\right)$ then the map $t \mapsto\langle f(t), u(t)\rangle$ is absolutely continuous in $(0, T)$ and for a.e. $t \in(0, T)$ it holds

$$
\frac{d}{d t}\langle f(t), u(t)\rangle=\langle\dot{f}(t), u(t)\rangle+\langle f(t), \dot{u}(t)\rangle .
$$

We already know, by Theorem 2.3, that the stored energy $t \mapsto \mathcal{E}(u(t))$ is absolutely continuous and that for a.e. $t \in(0, T)$ we have

$$
\dot{\mathcal{E}}(u(t))=-\frac{1}{2}|\dot{u}(t)|_{L_{+}^{2}}^{2}-\frac{1}{2}|\partial \mathcal{F}|_{L_{+}^{2}}^{2}(t, u(t)) d t+\langle f(t), \dot{u}(t)\rangle .
$$

Therefore $t \mapsto \mathcal{F}(t, u(t))=\mathcal{E}(u(t))-\langle f(t), u(t)\rangle$ is absolutely continuous and for a.e. $t \in(0, T)$

$$
\dot{\mathcal{F}}(t, u(t))=-\frac{1}{2}|\dot{u}(t)|_{L_{+}^{2}}^{2}-\frac{1}{2}|\partial \mathcal{F}|_{L_{+}^{2}}^{2}(t, u(t)) d t-\langle\dot{f}(t), u(t)\rangle,
$$

which gives (2.12).

\subsection{Characterization by differential inclusions in $\boldsymbol{H}_{0}^{\mathbf{1}}$}

The results of this section are essentially an adaption of those contained in [18]; we provide some short alternative proofs, for sake of completeness.

Proposition 6.1. Let $u_{0} \in H_{0}^{1}$ and $f \in A C\left(0, T ; L^{2}\right)$ then the unilateral gradient flow $u$ belongs to $W_{l o c}^{1, \infty}\left(0, T ; L^{2}\right) \cap W_{l o c}^{1,2}\left(0, T ; H_{0}^{1}\right)$.

Proof. We will employ the discrete evolutions $u_{n}$ obtained by the implicit Euler scheme of Sect. 4.1. We will show that given $0<T^{\prime}<T$ the sequence $u_{n}$ is bounded in $W^{1, \infty}\left(T^{\prime}, T ; L^{2}\right) \cap W^{1,2}\left(T^{\prime}, T ; H_{0}^{1}\right)$.

Let us denote $\dot{u}_{n, k}=\left(u_{n, k}-u_{n, k-1}\right) / \tau_{n}$. For $k \geq 0$ by (4.3) we have

$$
\left\|\dot{u}_{n, k+1}\right\|_{L^{2}}^{2}+d \mathcal{F}_{n}\left(t_{n, k+1}, u_{n, k+1}\right)\left[\dot{u}_{n, k+1}\right]=0 .
$$

For $k \geq 1$ by (4.4) we get

$$
d \mathcal{F}_{n}\left(t_{n, k}, u_{n, k}\right)\left[\dot{u}_{n, k+1}\right]+\left\langle\dot{u}_{n, k}, \dot{u}_{n, k+1}\right\rangle \geq 0 .
$$

Hence, for $k \geq 1$ we obtain

$$
\begin{aligned}
& d \mathcal{F}_{n}\left(t_{n, k}, u_{n, k}\right)\left[\dot{u}_{n, k+1}\right]-d \mathcal{F}_{n}\left(t_{n, k+1}, u_{n, k+1}\right)\left[\dot{u}_{n, k+1}\right] \\
& \quad \geq-\left\langle\dot{u}_{n, k}, \dot{u}_{n, k+1}\right\rangle+\left\langle\dot{u}_{n, k+1}, \dot{u}_{n, k+1}\right\rangle .
\end{aligned}
$$

Let us write explicitly the left hand side as

$$
\begin{aligned}
& a\left(u_{n, k}, \dot{u}_{n, k+1}\right)-\left\langle f_{n, k}, \dot{u}_{n, k+1}\right\rangle-a\left(u_{n, k+1}, \dot{u}_{n, k+1}\right)+\left\langle f_{n, k+1}, \dot{u}_{n, k+1}\right\rangle \\
& \quad=-\tau_{n} a\left(\dot{u}_{n, k+1}, \dot{u}_{n, k+1}\right)+\left\langle f_{n, k+1}-f_{n, k}, \dot{u}_{n, k+1}\right\rangle .
\end{aligned}
$$

A simple algebraic calculation gives $-\left\langle\dot{u}_{n, k}, \dot{u}_{n, k+1}\right\rangle+\left\langle\dot{u}_{n, k+1}, \dot{u}_{n, k+1}\right\rangle$ $\geq \frac{1}{2}\left\|\dot{u}_{n, k+1}\right\|_{L^{2}}^{2}-\frac{1}{2}\left\|\dot{u}_{n, k}\right\|_{L^{2}}^{2}$. Hence by coercivity (6.2) reads, for $k \geq 1$,

$$
\begin{aligned}
& -c \tau_{n}\left\|\dot{u}_{n, k+1}\right\|_{H_{0}^{1}}^{2}+\left\|f_{n, k+1}-f_{n, k}\right\|_{L^{2}}\left\|\dot{u}_{n, k+1}\right\|_{L^{2}} \\
& \quad \geq \frac{1}{2}\left\|\dot{u}_{n, k+1}\right\|_{L^{2}}^{2}-\frac{1}{2}\left\|\dot{u}_{n, k}\right\|_{L^{2}}^{2} .
\end{aligned}
$$

Neglecting the $H_{0}^{1}$-norm and denoting $a_{k}=\left\|\dot{u}_{n, k}\right\|_{L^{2}}$ we obtain the discrete inequality $a_{k+1}^{2}-a_{k}^{2} \leq b_{k} a_{k+1}$ where $b_{k}=2\left\|f_{n, k+1}-f_{n, k}\right\|_{L^{2}}$. By an elementary 
algebraic calculation we get $a_{k+1} \leq a_{k}+b_{k}$. Then for every $k_{0}<k$ we can write

$$
a_{k} \leq a_{k_{0}}+\sum_{j=k_{0}}^{k-1} b_{j} \leq a_{k_{0}}+2\|f\|_{A C\left(0, T ; L^{2}\right)} \leq a_{k_{0}}+C .
$$

Let $k_{n}^{\prime}$ such that $\tau_{n}\left(k_{n}^{\prime}-1\right)<T^{\prime} \leq \tau_{n} k_{n}^{\prime}$. Given $k>k_{n}^{\prime}$ the estimate $a_{k} \leq a_{k_{0}}+$ $C$ holds for every index $k_{0}$ such that $1 \leq k_{0} \leq k_{n}^{\prime}$ with $C=2\|f\|_{A C\left(0, T ; L^{2}\right)}$. Taking the sum of $a_{k} \leq a_{k_{0}}+C$ for $k_{0}=1, \ldots, k_{n}^{\prime}$ (and $k$ fixed) and dividing by $k_{n}^{\prime}$ yields

$$
a_{k} \leq \frac{1}{k_{n}^{\prime}} \sum_{k_{0}=1}^{k_{n}^{\prime}}\left(a_{k_{0}}+C\right) \leq \frac{1}{T^{\prime}}\left(\sum_{k_{0}=1}^{k_{n}^{\prime}} \tau_{n} a_{k_{0}}\right)+C .
$$

Hence, for every $k>k_{n}^{\prime}$ we have

$$
\left\|\dot{u}_{n, k}\right\|_{L^{2}} \leq \frac{1}{T^{\prime}} \int_{0}^{T}\left\|\dot{u}_{n}(t)\right\|_{L^{2}} d t+C \leq C\left(T^{\prime}\right),
$$

where $C\left(T^{\prime}\right)>0$ is independent of $n$ because the sequence $u_{n}$ is bounded in $H^{1}\left(0, T ; L^{2}\right)$. (Note that $C\left(T^{\prime}\right)$ diverges as $\left.T^{\prime} \rightarrow 0^{+}\right)$. Taking the supremum with respect to $k>k_{n}^{\prime}$ it follows that the sequence $\dot{u}_{n}$ is bounded in $L^{\infty}\left(T^{\prime \prime}, T ; L^{2}\right)$ for every $T^{\prime \prime}>T^{\prime}>0$.

Let us go back to (6.3). For every $k>k_{n}^{\prime}$, now we can write

$$
\frac{1}{2}\left\|\dot{u}_{n, k}\right\|_{L^{2}}^{2}-\frac{1}{2}\left\|\dot{u}_{n, k+1}\right\|_{L^{2}}^{2}+C\left(T^{\prime}\right)\left\|f_{n, k}-f_{n, k-1}\right\|_{L^{2}} \geq c \tau_{n}\left\|\dot{u}_{n, k+1}\right\|_{H_{0}^{1}}^{2} .
$$

Taking the sum for $k>k_{n}^{\prime}$ we get

$$
\begin{aligned}
c \int_{T^{\prime \prime}}^{T}\left\|\dot{u}_{n}\right\|_{H_{0}^{1}}^{2} d t & \leq c \int_{\tau_{n} k_{n}^{\prime}}^{T}\left\|\dot{u}_{n}\right\|_{H_{0}^{1}}^{2} d t \\
& \leq C\left(T^{\prime}\right)\|f\|_{A C\left(0, T ; L^{2}\right)}+\frac{1}{2}\left\|\dot{u}_{n, k_{n}^{\prime}+1}\right\|_{L^{2}}^{2} \leq C^{\prime}\left(T^{\prime}\right),
\end{aligned}
$$

for every $T^{\prime \prime}>T^{\prime}>0$, which concludes the proof.

Let $\Phi: H_{0}^{1} \rightarrow[0,+\infty]$ be defined by $\Phi(u)=\frac{1}{2}\|u\|_{L^{2}}^{2}+I_{+}(u)$ (where $I_{+}(u)$ denotes the indicator function of $\left.\{u>0\}\right)$ and let $\partial \Phi(u) \subset H^{-1}$ be its subdifferential. Let $\Phi^{*}$ be its Legendre transform, i.e. $\Phi^{*}(\xi)=\sup \{(\xi, v)-$ $\left.\Phi(v): v \in H_{0}^{1}\right\}$ for $\xi \in H^{-1}$. By Lemma 3.6 it is easy to check that the domain of $\Phi^{*}$ is $D \Phi^{*}=\left\{\xi \in H^{-1}: \xi \in \mathcal{M}_{l o c}, \xi_{+} \in L^{2}\right\}$ and then that $\Phi^{*}(\xi)=\frac{1}{2}\left\|\xi_{+}\right\|_{L^{2}}^{2}$ for $\xi \in D \Phi^{*}$.

Proposition 6.2. Let $f \in A C\left(0, T ; L^{2}\right)$ and $u \in W_{l o c}^{1,2}\left(0, T ; H_{0}^{1}\right)$. Then $u$ is a unilateral gradient flow for $\mathcal{F}$ with initial condition $u_{0}$ if and only if it solves the differential inclusion

$$
\left\{\begin{array}{l}
\partial \Phi(\dot{u}(t)) \ni A u(t)+f(t) \quad \text { in } H^{-1} \text { for a.e. } t \in(0, T) \\
u(0)=u_{0} .
\end{array}\right.
$$


Proof. Since $\dot{u}(t) \in H_{0}^{1}$, for a.e. $t \in(0, T)$, by classical results in convex analysis we have

$$
\begin{gathered}
A u(t)+f(t) \in \partial \Phi(\dot{u}(t)) \\
\mathbb{1} \\
\Phi(\dot{u}(t))+\Phi^{*}(A u(t)+f(t))=(A u(t)+f(t), \dot{u}(t)) \\
\mathbb{\Downarrow} \\
\frac{1}{2}\|\dot{u}(t)\|_{L^{2}}^{2}+\frac{1}{2}\left\|[A u(t)+f(t)]_{+}\right\|_{L^{2}}^{2}=-d \mathcal{F}(t, u(t))[\dot{u}(t)] \\
\mathbb{\mathbb { }} \\
\frac{1}{2}|\dot{u}(t)|_{L_{+}^{2}}^{2}+\frac{1}{2}|\partial \mathcal{F}|_{L_{+}^{2}}^{2}(t, u(t))=-d \mathcal{F}(t, u(t))[\dot{u}(t)] .
\end{gathered}
$$

The fact that $\partial \Phi(\dot{u}(t)) \neq \emptyset$ implicitly says that $\dot{u}(t) \in D \Phi$ and hence $\dot{u}(t) \geq 0$. Moreover, if $u \in W_{l o c}^{1,2}\left(0, T ; H_{0}^{1}\right)$ with $\dot{u} \geq 0$ then by (6.4) for a.e. $t \in(0, T)$ we can write

$$
\begin{aligned}
\dot{\mathcal{F}}(t, u(t)) & =d \mathcal{F}(t, u(t))[\dot{u}(t)]+\partial_{t} \mathcal{F}(t, u(t)) \\
& \leq-\frac{1}{2}|\dot{u}(t)|_{L_{+}^{2}}^{2}-\frac{1}{2}|\partial \mathcal{F}|_{L_{+}^{2}}^{2}(t, u(t))-\langle\dot{f}(t), u(t)\rangle,
\end{aligned}
$$

which is indeed the characterization (2.12) of unilateral gradient flows. Conversely, if the energy identity (2.14) holds then for a.e. $t \in(0, T)$ we have

$$
\begin{aligned}
\dot{\mathcal{F}}(t, u(t)) & =d \mathcal{F}(t, u(t))[\dot{u}(t)]+\partial_{t} \mathcal{F}(t, u(t)) \\
& =-\frac{1}{2}|\dot{u}(t)|_{L_{+}^{2}}^{2}-\frac{1}{2}|\partial \mathcal{F}|_{L_{+}^{2}}^{2}(t, u(t))-\langle\dot{f}(t), u(t)\rangle
\end{aligned}
$$

and then (6.4) holds.

\section{A characterization for $f$ independent of time}

In this section we will prove Proposition 2.13.

Step I. We employ the discrete scheme of Sect.4.1. We claim that for every $v \in H_{0}^{1}$ with $u_{0} \leq v \leq u_{n, k+1}$ we have

$$
\mathcal{F}\left(u_{n, k+1}\right)+\frac{1}{2 \tau_{n}}\left\|u_{n, k+1}-u_{n, k}\right\|_{L^{2}}^{2} \leq \mathcal{F}(v)+\frac{1}{2 \tau_{n}}\left\|v-u_{n, k}\right\|_{L^{2}}^{2} .
$$

Given $v \in H_{0}^{1}$ with $u_{0} \leq v \leq u_{n, k+1}$ and $0 \leq m \leq k+1$ let $\Omega_{n, m}=\left\{v \geq u_{n, m}\right\}$. The monotonicity of $u_{n, m}$ with respect to $m$ implies that the sets $\Omega_{n, m}$ are monotone non-increasing, moreover $\Omega_{n, 0}=\Omega$ and $\Omega_{n, k+1}=\left\{v=u_{n, k+1}\right\}$. Note that $\left\{\Omega_{n, m} \backslash \Omega_{n, m+1}\right.$ : for $\left.m=0, \ldots, k\right\}$ is a disjoint partition of $\Omega \backslash \Omega_{n, k+1}$. For any measurable subset $\Omega^{\prime}$ of $\Omega$ we employ the notation

$$
\mathcal{F}_{\Omega^{\prime}}(w)=\int_{\Omega^{\prime}} \nabla w \cdot B \nabla w+b w^{2}-f w d x .
$$

For each index $0 \leq m \leq k$ let us define $v_{n, m}=\min \left\{\max \left\{v, u_{n, m}\right\}, u_{n, m+1}\right\}$, i.e.,

$$
v_{n, m}= \begin{cases}u_{n, m+1} & \text { in } \Omega_{n, m+1} \\ v & \text { in } \Omega_{n, m} \backslash \Omega_{n, m+1} \\ u_{n, m} & \text { in } \Omega_{n, m}^{c}\end{cases}
$$


Since $u_{n, m} \leq v_{n, m} \leq u_{n, m+1}$ by minimality we have

$$
\mathcal{F}\left(u_{n, m+1}\right)+\frac{1}{2 \tau_{n}}\left\|u_{n, m+1}-u_{n, m}\right\|_{L^{2}}^{2} \leq \mathcal{F}\left(v_{n, m}\right)+\frac{1}{2 \tau_{n}}\left\|v_{n, m}-u_{n, m}\right\|_{L^{2}}^{2} .
$$

Being $\left\|u_{n, m+1}-u_{n, m}\right\|_{L^{2}}^{2} \geq\left\|v_{n, m}-u_{n, m}\right\|_{L^{2}}^{2}$ we have $\mathcal{F}\left(u_{n, m+1}\right) \leq \mathcal{F}\left(v_{n, m}\right)$; the latter inequality can be written as

$$
\begin{aligned}
& \mathcal{F}_{\Omega_{n, m+1}}\left(u_{n, m+1}\right)+\mathcal{F}_{\Omega_{n, m+1}^{c}}\left(u_{n, m+1}\right) \\
& \quad \leq \mathcal{F}_{\Omega_{n, m+1}}\left(v_{n, m}\right)+\mathcal{F}_{\Omega_{n, m} \backslash \Omega_{n, m+1}}\left(v_{n, m}\right)+\mathcal{F}_{\Omega_{n, m}^{c}}\left(v_{n, m}\right)
\end{aligned}
$$

which, by definition of $v_{n, m}$, yields

$$
\mathcal{F}_{\Omega_{n, m+1}^{c}}\left(u_{n, m+1}\right) \leq \mathcal{F}_{\Omega_{n, m} \backslash \Omega_{n, m+1}}(v)+\mathcal{F}_{\Omega_{n, m}^{c}}\left(u_{n, m}\right) .
$$

Let $m=k+1$. Being $\Omega_{n, k+1}=\left\{v=u_{n, k+1}\right\}$ we have $v_{n, k}=v$ in $\Omega_{n, k}$ (while $v_{n, k}=u_{n, k}$ in $\left.\Omega_{n, k}^{c}\right)$, hence by minimality we can write

$$
\begin{aligned}
\mathcal{F}\left(u_{n, k+1}\right) & +\frac{1}{2 \tau_{n}}\left\|u_{n, k+1}-u_{n, k}\right\|_{L^{2}}^{2} \leq \mathcal{F}\left(v_{n, k}\right)+\frac{1}{2 \tau_{n}}\left\|v_{n, k}-u_{n, k}\right\|_{L^{2}}^{2} \\
& \leq \mathcal{F}_{\Omega_{n, k}}(v)+\mathcal{F}_{\Omega_{n, k}^{c}}\left(u_{n, k}\right)+\frac{1}{2 \tau_{n}} \int_{\Omega_{n, k}}\left|v-u_{n, k}\right|^{2} d x .
\end{aligned}
$$

Using iteratively (7.2) we get

$\mathcal{F}_{\Omega_{n, k}^{c}}\left(u_{n, k}\right) \leq \sum_{m=0}^{k-1} \mathcal{F}_{\Omega_{n, m} \backslash \Omega_{n, m+1}}(v)=\mathcal{F}_{\Omega_{n, 0}}(v)-\mathcal{F}_{\Omega_{n, k}}(v)=\mathcal{F}(v)-\mathcal{F}_{\Omega_{n, k}}(v)$.

Hence

$$
\mathcal{F}\left(u_{n, k+1}\right)+\frac{1}{2 \tau_{n}}\left\|u_{n, k+1}-u_{n, k}\right\|_{L^{2}}^{2} \leq \mathcal{F}(v)+\frac{1}{2 \tau_{n}} \int_{\Omega_{n, k}}\left|v-u_{n, k}\right|^{2} d x,
$$

which proves the claim (7.1).

Step II. Thanks to (7.1), the incremental problem (4.1) can be replaced by the fixed obstacle problem

$$
u_{n, k+1} \in \operatorname{argmin}\left\{\mathcal{F}(u)+I_{+}\left(u-u_{0}\right)+\frac{1}{2 \tau_{n}}\left\|u-u_{n, k}\right\|_{L^{2}}^{2}: u \in H_{0}^{1}\right\} .
$$

Indeed, let $v \geq u_{0}$. Denote $v^{+}=\max \left\{v, u_{n, k+1}\right\}$ and $v^{-}=\min \left\{v, u_{n, k+1}\right\}$, accordingly let $\Omega^{+}=\left\{v>u_{n, k+1}\right\}$ and $\Omega^{-}=\Omega \backslash \Omega^{+}$. By minimality of $u_{n, k+1}$ we can write

$$
\mathcal{F}\left(u_{n, k+1}\right)+\frac{1}{2 \tau_{n}}\left\|u_{n, k+1}-u_{n, k}\right\|_{L^{2}}^{2} \leq \mathcal{F}\left(v^{+}\right)+\frac{1}{2 \tau_{n}}\left\|v^{+}-u_{n, k}\right\|_{L^{2}}^{2},
$$

then, being $v^{+}=u_{n, k+1}$ in $\Omega^{-}$, we have

$$
\mathcal{F}_{\Omega^{+}}\left(u_{n, k+1}\right)+\frac{1}{2 \tau_{n}}\left\|u_{n, k+1}-u_{n, k}\right\|_{L^{2}\left(\Omega^{+}\right)}^{2} \leq \mathcal{F}_{\Omega^{+}}(v)+\frac{1}{2 \tau_{n}}\left\|v-u_{n, k}\right\|_{L^{2}\left(\Omega^{+}\right)}^{2} .
$$

Since $u_{0} \leq v^{-} \leq u_{n, k+1}$ we can invoke (7.1) which gives

$$
\mathcal{F}\left(u_{n, k+1}\right)+\frac{1}{2 \tau_{n}}\left\|u_{n, k+1}-u_{n, k}\right\|_{L^{2}}^{2} \leq \mathcal{F}\left(v^{-}\right)+\frac{1}{2 \tau_{n}}\left\|v^{-}-u_{n, k}\right\|_{L^{2}}^{2} .
$$

In $\Omega^{+}$we have $v^{-}=u_{n, k+1}$, and then

$$
\mathcal{F}_{\Omega^{-}}\left(u_{n, k+1}\right)+\frac{1}{2 \tau_{n}}\left\|u_{n, k+1}-u_{n, k}\right\|_{L^{2}\left(\Omega^{-}\right)}^{2} \leq \mathcal{F}_{\Omega^{-}}(v)+\frac{1}{2 \tau_{n}}\left\|v-u_{n, k}\right\|_{L^{2}\left(\Omega^{-}\right)}^{2} .
$$

Taking the sum of estimates in $\Omega^{ \pm}$shows that (7.3) holds. As a consequence, the limit evolution $u$ turns out to be the $L^{2}$-gradient flow for the functional $\tilde{\mathcal{F}}(u)=\mathcal{F}(u)+I_{+}\left(u-u_{0}\right)$. 
Step III. Now, let us show that the unilateral gradient flow is a solution of the parabolic obstacle problem (2.15), i.e.,

$$
\begin{cases}\dot{u}(t)-A u(t)-f \geq 0 & \text { in } H^{-1} \text { for a.e. } t \in(0, T) \\ \left(u(t)-u_{0}, \dot{u}(t)-A u(t)-f\right)=0 & \text { for a.e. } t \in(0, T) \\ u(0)=u_{0}, \quad u(t) \geq u_{0} & \text { for a.e. } t \in(0, T)\end{cases}
$$

By Theorem 2.5 we know that $\dot{u}(t)=[A u(t)+f]_{+}$hence

$$
\dot{u}(t)-(A u(t)+f)=\dot{u}(t)-[A u(t)+f]_{+}+[A u(t)+f]_{-}=[A u(t)+f]_{-} \geq 0 \text {. }
$$

In particular $\left(u(t)-u_{0}, \dot{u}(t)-A u(t)-f\right) \geq 0$. Therefore, re-writing the second line in (7.4), it remains to show that for a.e. $t \in(0, T)$ we have

$$
\left\langle u(t)-u_{0}, \dot{u}(t)\right\rangle+d \mathcal{F}(u(t))\left[u(t)-u_{0}\right] \leq 0 .
$$

The functional $\mathcal{J}(w)=\mathcal{F}(w)+\frac{1}{2 \tau_{n}}\left\|w-u_{n, k}\right\|_{L^{2}}^{2}$ is convex, thus (7.1) implies that $d \mathcal{J}\left(u_{n, k+1}\right)\left[u_{0}-u_{n, k+1}\right] \geq 0$, i.e.

$$
d \mathcal{F}\left(u_{n, k+1}\right)\left[u_{0}-u_{n, k+1}\right]+\frac{1}{\tau_{n}}\left\langle u_{n, k+1}-u_{n, k}, u_{0}-u_{n, k+1}\right\rangle \geq 0
$$

In terms of the piece-wise affine interpolation $u_{n}$ and the piece-wise constant interpolation $u_{n}^{\sharp}$ (see Sect. 4.1), for every $t \in\left(t_{n, k}, t_{n, k+1}\right)$ the previous inequality reads

$$
d \mathcal{F}\left(u_{n}^{\sharp}(t)\right)\left[u_{0}-u_{n}^{\sharp}(t)\right]+\left\langle\dot{u}_{n}(t), u_{0}-u_{n}^{\sharp}(t)\right\rangle \geq 0 .
$$

Given $0<t^{-}<t^{+}<T$, we obtain

$$
\int_{t^{-}}^{t^{+}} d \mathcal{F}\left(u_{n}^{\sharp}(t)\right)\left[u_{0}-u_{n}^{\sharp}(t)\right]+\left\langle\dot{u}_{n}(t), u_{0}-u_{n}^{\sharp}(t)\right\rangle d t \geq 0 .
$$

By Remark 4.2 we know that $u_{n} \rightarrow u$ in $H^{1}\left(0, T ; L^{2}\right)$, that $u_{n}^{\sharp}$ is bounded in $L^{\infty}\left(0, T ; H_{0}^{1}\right)$ and that $u_{n}^{\sharp}(t) \rightarrow u(t)$ strongly in $H_{0}^{1}$ for a.e. $t$ in $(0, T)$. Therefore, we can pass to the limit in the previous inequality and get

$$
\int_{t^{-}}^{t^{+}} d \mathcal{F}(u(t))\left[u_{0}-u(t)\right]+\left\langle\dot{u}(t), u_{0}-u(t)\right\rangle d t \geq 0 .
$$

By arbitrariness of $t^{-}$and $t^{+}$we conclude that (7.5) holds a.e. in $[0, T]$.

Step IV. We prove that there exists a unique solution of (7.4). Assume, by contradiction, that $u_{\mathrm{I}}$ and $u_{\mathrm{II}}$ are solutions of (7.4) with $u_{\mathrm{I}}\left(t^{*}\right) \neq u_{\mathrm{II}}\left(t^{*}\right)$. We define $u_{\natural}=\frac{1}{2}\left(u_{\mathrm{I}}+u_{\mathrm{II}}\right)$. By linearity, for a.e. $t \in(0, T)$ we have $\dot{u}_{\natural}(t)-A u_{\natural}(t)-$ $f \geq 0$ and $u_{\natural}(t) \geq u_{0}$, thus

$$
\int_{0}^{t^{*}}\left(u_{\natural}(t)-u_{0}, \dot{u}_{\natural}(t)-A u_{\natural}(t)-f\right) d t \geq 0 .
$$

On the other hand, by the strict convexity of the $L^{2}$-norm we can write

$$
\begin{aligned}
\int_{0}^{t^{*}}\left(u_{\natural}(t)-u_{0}, \dot{u}_{\natural}(t)\right) d t & =\frac{1}{2}\left\|u_{\natural}\left(t^{*}\right)-u_{0}\right\|_{L^{2}}^{2} \\
& <\frac{1}{2}\left(\frac{1}{2}\left\|u_{\mathrm{I}}\left(t^{*}\right)-u_{0}\right\|_{L^{2}}^{2}+\frac{1}{2}\left\|u_{\mathrm{II}}\left(t^{*}\right)-u_{0}\right\|_{L^{2}}^{2}\right) \\
& =\frac{1}{2} \int_{0}^{t^{*}}\left(u_{\mathrm{I}}(t)-u_{0}, \dot{u}_{\mathrm{I}}(t)\right)+\left(u_{\mathrm{II}}(t)-u_{0}, \dot{u}_{\mathrm{II}}(t)\right) d t .
\end{aligned}
$$


Moreover, by convexity of $u \mapsto a(u, u)$ we have

$$
\begin{aligned}
\left(u_{\natural}(t)-u_{0},-A u_{\natural}(t)-f\right)= & \left(u_{\natural}(t),-A u_{\natural}(t)\right)-\left(u_{\natural}(t), f\right)+\left(u_{0}, A u_{\natural}(t)+f\right) \\
= & a\left(u_{\text {七}}(t), u_{\natural}(t)\right)-\left(u_{\natural}(t), f\right)+\left(u_{0}, A u_{\natural}(t)+f\right) \\
\leq & \frac{1}{2} a\left(u_{\mathrm{I}}(t), u_{\mathrm{I}}(t)\right)+\frac{1}{2} a\left(u_{\mathrm{II}}(t), u_{\mathrm{II}}(t)\right) \\
& -\frac{1}{2}\left(u_{\mathrm{I}}(t), f\right)-\frac{1}{2}\left(u_{\mathrm{II}}(t), f\right) \\
& +\frac{1}{2}\left(u_{0}, A u_{\mathrm{I}}(t)+f\right)+\frac{1}{2}\left(u_{0}, A u_{\mathrm{II}}(t)+f\right) \\
= & \frac{1}{2}\left(u_{\mathrm{I}}(t)-u_{0},-A u_{\mathrm{I}}(t)-f\right) \\
& +\frac{1}{2}\left(u_{\mathrm{II}}(t)-u_{0},-A u_{\mathrm{II}}(t)-f\right)
\end{aligned}
$$

Taking the integral in $\left(0, t^{*}\right)$ we obtain the contradiction

$$
\begin{aligned}
& \int_{0}^{t^{*}}\left(u_{\natural}(t)-u_{0}, \dot{u}_{\natural}(t)-A u_{\natural}(t)-f\right) d t \\
& <\frac{1}{2} \int_{0}^{t^{*}}\left(u_{\mathrm{I}}(t)-u_{0}, \dot{u}_{\mathrm{I}}(t)-A u_{\mathrm{I}}(t)-f\right) d t \\
& +\int_{0}^{t^{*}}\left(u_{\mathrm{II}}(t)-u_{0}, \dot{u}_{\mathrm{II}}(t)-A u_{\mathrm{II}}(t)-f\right) d t=0,
\end{aligned}
$$

which concludes the proof.

Remark 7.1. If $f$ depends on time then, in general, unilateral gradient flows do not enjoy $(2.15)$, as the following example shows. Let $\Omega=(0,1)$ and $u_{0}(x)=$ $x(x-1)$. Define

$$
u(t)=\left\{\begin{array}{ll}
(1-t) u_{0} & \text { if } t \in[0,1] \\
0 & \text { if } t>1,
\end{array} \quad f(t)= \begin{cases}-u_{0}-2 & \text { if } t \in[0,1] \\
u_{0} & \text { if } t>1\end{cases}\right.
$$

It is easy to check that $u$ is monotone non-decreasing and that $\dot{u}(t)=u^{\prime \prime}(t)+$ $f(t)$ for $t \in(0,1)$. In particular we have $\|\dot{u}(t)\|_{L^{2}}=|\dot{u}(t)|_{L_{+}^{2}}$ and $|\partial \mathcal{F}|_{L_{+}^{2}}(t, u(t))=$ $\left\|u^{\prime \prime}(t)+f(t)\right\|_{L^{2}}$. Thus, by the chain rule

$\dot{\mathcal{F}}(t, u(t))=\partial_{u} \mathcal{F}(t, u(t))[\dot{u}(t)]+\partial_{t} \mathcal{F}(t, u(t))=d \mathcal{F}(u(t))[\dot{u}(t)]-\langle\dot{f}(t), u(t)\rangle$,

and

$$
\begin{aligned}
d \mathcal{F}(t, u(t))[\dot{u}(t)] & =-\int_{\Omega}\left(u^{\prime \prime}(t)+f(t)\right) \dot{u}(t) d x \\
& =-\frac{1}{2}\|\dot{u}(t)\|_{L^{2}}^{2}-\frac{1}{2}\left\|u^{\prime \prime}(t)+f(t)\right\|_{L^{2}}^{2} .
\end{aligned}
$$

Hence $u$ is the unilateral gradient flow for $t \in[0,1]$. For $t>1$ we have $\dot{\mathcal{F}}(t, u(t))=0$ and $u(t)=0$, hence $\|\dot{u}(t)\|_{L^{2}}=0$. In this case we have $|\partial \mathcal{F}|_{L_{+}^{2}}(t, u(t))=\left\|\left[u^{\prime \prime}(t)+f(t)\right]_{+}\right\|_{L^{2}}=0$. Hence $u$ is the unilateral gradient flow also for $t>1$. On the other hand for $t>1$ we have

$$
\left(u(t)-u_{0}, \dot{u}(t)-u^{\prime \prime}(t)-f\right)=\int_{(0,1)} u_{0}^{2} d x \neq 0,
$$

thus (2.15) does not hold.

Funding Open access funding provided by Università degli Studi di Pavia within the CRUI-CARE Agreement. 
Open Access. This article is licensed under a Creative Commons Attribution 4.0 International License, which permits use, sharing, adaptation, distribution and reproduction in any medium or format, as long as you give appropriate credit to the original author(s) and the source, provide a link to the Creative Commons licence, and indicate if changes were made. The images or other third party material in this article are included in the article's Creative Commons licence, unless indicated otherwise in a credit line to the material. If material is not included in the article's Creative Commons licence and your intended use is not permitted by statutory regulation or exceeds the permitted use, you will need to obtain permission directly from the copyright holder. To view a copy of this licence, visit http://creativecommons. org/licenses/by/4.0/.

Publisher's Note Springer Nature remains neutral with regard to jurisdictional claims in published maps and institutional affiliations.

\section{Appendix A. Metric settings}

Having in mind [5] it is interesting to see if and how our framework fits into some sort of metric setting. For sake of simplicity, we assume that $f$ is constant. A singular metric for $\boldsymbol{L}_{+}^{\mathbf{2}}$. Consider the "singular" metric

$$
d_{+}(v, u)= \begin{cases}\|v-u\|_{L^{2}} & \text { if } v \geq u \\ +\infty & \text { otherwise }\end{cases}
$$

Accordingly, we will say that $u_{m} \rightarrow u$ when $d_{+}\left(u_{m}, u\right) \rightarrow 0$, i.e. when $u_{m} \geq u$ and $u_{m} \rightarrow u$ in $L^{2}$.

It is clear that if $u \in A C_{l o c}\left(0, T ; L_{+}^{2}\right)$ then $u$ is monotone non-decreasing, moreover the metric derivative (cf. [5, Theorem 1.1.2]) exists a.e. in $(0, T)$ and reads

$$
|\dot{u}|_{L_{+}^{2}}(t)=\lim _{h \rightarrow 0^{+}} \frac{d_{+}(u(t+h), u(t))}{h}=\|\dot{u}(t)\|_{L^{2}}=|\dot{u}(t)|_{L_{+}^{2}} .
$$

Finally, the unilateral slope reads (cf. [5, Definition 1.2.4])

$$
|\partial \mathcal{F}|_{L_{+}^{2}}(u)=\limsup _{v \rightarrow u} \frac{[\mathcal{F}(v)-\mathcal{F}(u)]_{-}}{d_{+}(v, u)} .
$$

A key point in [5] is the fact that slopes are upper gradients (cf. [5, Definition 1.2.1 and 1.2.2]), however in our case we have the following.

Remark A.1. The unilateral slope $|\partial \mathcal{F}|_{L_{+}^{2}}$ is neither a strong nor a weak upper gradient for $\mathcal{F}$.

The counter-example of Sect. 5.2 applies also here. Let $u_{0}(x)=1-|x|$, $u(t)=(1+t) u_{0}$ and $f=u_{0}$. Clearly $\dot{u}=u_{0}$ and $u \in A C\left(0, T ; L^{2}(-1,1)\right)$. Moreover,

$$
\begin{aligned}
\mathcal{F}(u(t)) & =\frac{1}{2}\|u(t)\|_{H_{0}^{1}}^{2}-\langle f, u(t)\rangle=\frac{1}{2}(1+t)^{2}\left\|u_{0}\right\|_{H_{0}^{1}}^{2}-(1+t)\left\|u_{0}\right\|_{L^{2}}^{2} \\
& =\mathcal{F}\left(u_{0}\right)+\left(\frac{1}{2} t^{2}+t\right)\left\|u_{0}\right\|_{H_{0}^{1}}^{2}-t\left\|u_{0}\right\|_{L^{2}}^{2} .
\end{aligned}
$$

Hence,

$$
\dot{\mathcal{F}}(u(t))=(t+1)\left\|u_{0}\right\|_{H_{0}^{1}}^{2}-\left\|u_{0}\right\|_{L^{2}}^{2} .
$$


Now, let us check that the inequality $|\dot{\mathcal{F}}(u(t))| \leq|\partial \mathcal{F}|_{L_{+}^{2}}(u(t))|\dot{u}(t)|_{L_{+}^{2}}$ fails for $t$ sufficiently large. For every $z \in H_{0}^{1}(-1,1)$ with $z \geq 0$ and $\|z\|_{L^{2}} \leq 1$ we have

$$
-d \mathcal{F}(u(t))[z]=\left(u^{\prime \prime}(t)+f, z\right)=\left(-2(1+t) \delta_{0}+u_{0}, z\right) \leq\left\langle u_{0}, z\right\rangle \leq\left\|u_{0}\right\|_{L^{2}},
$$

then $|\partial \mathcal{F}|_{L_{+}^{2}}(u(t))=\sup \left\{-d \mathcal{F}(u(t))[z]: z \in H_{0}^{1},|z|_{L_{+}^{2}} \leq 1\right\} \leq\left\|u_{0}\right\|_{L^{2}}$ and thus

$$
|\partial \mathcal{F}|_{L_{+}^{2}}(u(t))|\dot{u}(t)|_{L_{+}^{2}} \leq\left\|u_{0}\right\|_{L^{2}}^{2}
$$

A quasi-metric for $\boldsymbol{L}_{\boldsymbol{\alpha}}^{\mathbf{2}}$. In the setting of Sect. 4.3 it is natural to introduce

$$
d_{\alpha}(u, v)=|u-v|_{L_{\alpha}^{2}}=\left(\int_{\Omega} \psi_{\alpha}(u-v) d x\right)^{1 / 2}
$$

where

$$
\psi_{\alpha}(u-v)= \begin{cases}(u-v)^{2} & \text { if } u \geq v \\ \alpha(u-v)^{2} & \text { if } u<v\end{cases}
$$

It is not difficult to check that $d_{\alpha}$ is a quasi-metric in $L^{2}$, i.e., that

$$
d_{\alpha}(u, v) \geq 0, \quad d_{\alpha}(u, v)=0 \Leftrightarrow u=v, \quad d_{\alpha}(u, v) \leq d_{\alpha}(u, z)+d_{\alpha}(z, v) ;
$$

however $d_{\alpha}$ is not a metric since it is not symmetric. First, note that the metric derivative coincides with $|\dot{v}|_{L_{\alpha}^{2}}$; indeed, if $v \in A C\left(0, T ; L^{2}\right)$ then, in every differentiability point $t$, we can write

$$
\begin{aligned}
\lim _{h \rightarrow 0} \frac{d_{\alpha}(v(t+h), v(t))}{h} & =\lim _{h \rightarrow 0}\left(\int_{\Omega} \frac{\psi_{\alpha}(v(t+h)-v(t))}{h^{2}} d x\right)^{1 / 2} \\
& =\lim _{h \rightarrow 0}\left(\int_{\Omega} \psi_{\alpha}\left(\frac{v(t+h)-v(t)}{h}\right) d x\right)^{1 / 2} \\
& =\left(\int_{\Omega} \psi_{\alpha}(\dot{v}(t)) d x\right)^{1 / 2}=|\dot{v}|_{L_{\alpha}^{2}}
\end{aligned}
$$

Therefore the slope of $\mathcal{F}(t, \cdot)$ with respect to the quasi-metric $d_{\alpha}$ actually coincides with the slope $|\partial \mathcal{F}|_{L_{\alpha}^{2}}(t, \cdot)$ defined in Sect. 4.3. In this case, following the arguments of $\left[5\right.$, Theorem 1.2.5], the slope $|\partial \mathcal{F}|_{L_{\alpha}^{2}}(t, \cdot)$ turns out to be a strong upper gradient for $\mathcal{F}(t, \cdot)$.

\section{Appendix B. A unilateral $L^{2}$-subdifferential}

In this section we propose a notion of unilateral subdifferential in $L^{2}$ and show its connection with the unilateral slope (2.4). For sake of simplicity we consider again an autonomous functional $\mathcal{F}$.

Definition B.1. For $u \in H_{0}^{1}$ the unilateral subdifferential is

$$
\partial_{+} \mathcal{F}(u):=\left\{\xi \in L^{2}: \mathcal{F}(v) \geq \mathcal{F}(u)+\langle\xi, v-u\rangle \text { for } v \in L^{2}, v \geq u\right\} .
$$


As usual, $\partial_{+} \mathcal{F}(u)=\emptyset$ if $\mathcal{F}(u)=+\infty$ (i.e. if $u \notin H_{0}^{1}$ ). It is easy to check that $\partial_{+} \mathcal{F}(u)$ is convex and closed. Note also that $\partial_{+} \mathcal{F}(u)$ is much larger than $\partial \mathcal{F}(u)$, indeed if $\zeta \in \partial \mathcal{F}(u)$ then every $\xi \geq \zeta$ belongs to $\partial_{+} \mathcal{F}(u)$. However, in analogy with the unconstrained setting, we can define the "minimal selection"

$$
\partial_{+}^{\circ} \mathcal{F}(u)=\operatorname{argmin}\left\{\|\xi\|_{L^{2}}: \xi \in \partial_{+} \mathcal{F}(u)\right\} .
$$

Note that, being $\partial_{+} \mathcal{F}(u)$ closed in $L^{2}$, the minimum is attained. Next lemma shows the natural relationship between unilateral slope and unilateral subdifferential.

Lemma B.2. If $u \in H_{0}^{1}$ then $|\partial \mathcal{F}|_{L_{+}^{2}}(u)=\min \left\{\|\xi\|_{L^{2}}: \xi \in \partial_{+} \mathcal{F}(u)\right\}$. Moreover, if $\partial_{+} \mathcal{F}(u) \neq \emptyset$ then $\partial_{+}^{\circ} \mathcal{F}(u)=\left\{-[A u+f]_{+}\right\}$.

Proof. In the definition of slope (2.4) it is not restrictive to consider $v \geq u$ such that $\mathcal{F}(v) \leq \mathcal{F}(u)$. In this case, for $\xi \in \partial_{+} \mathcal{F}(u)$ we have

$$
[\mathcal{F}(v)-\mathcal{F}(u)]_{-}=\mathcal{F}(u)-\mathcal{F}(v) \leq-\langle\xi, v-u\rangle \leq\|\xi\|_{L^{2}}\|v-u\|_{L^{2}} .
$$

Hence $|\partial \mathcal{F}|_{L_{+}^{2}}(u) \leq\|\xi\|_{L^{2}}$ for every $\xi \in \partial_{+} \mathcal{F}(u)$ from which

$$
|\partial \mathcal{F}|_{L_{+}^{2}}(u) \leq \min \left\{\|\xi\|_{L^{2}}: \xi \in \partial_{+} \mathcal{F}(u)\right\} .
$$

The previous inequality holds also when $\partial_{+} \mathcal{F}(u)=\emptyset$, in which case the righthand side is infinite.

Let us prove that $|\partial \mathcal{F}|_{L_{+}^{2}}(u) \geq \min \left\{\|\xi\|_{L^{2}}: \xi \in \partial_{+} \mathcal{F}(u)\right\}$. If $|\partial \mathcal{F}|_{L_{+}^{2}}(u)$ is infinite there is nothing to prove. Otherwise, $u \in H_{0}^{1}$ and by Lemma 3.1

$$
|\partial \mathcal{F}|_{L_{+}^{2}}(u)=\sup \left\{-d \mathcal{F}(u)[z]: z \in H_{0}^{1}, z \geq 0,\|z\|_{L^{2}} \leq 1\right\}<+\infty .
$$

By Lemma 3.6 it follows that $-d \mathcal{F}(u)=A u+f$ is a Radon measure $\mu$ with positive part $\mu_{+}$in $L^{2}$ and $|\partial \mathcal{F}|_{L_{+}^{2}}(u)=\left\|\mu_{+}\right\|_{L^{2}}$. To conclude, it is enough to see that $-[A u+f]_{+}=-\mu_{+} \in \partial_{+} \mathcal{F}(u)$; indeed, by convexity for every $v \in H_{0}^{1}$ with $v \geq u$ we have

$$
\mathcal{F}(u)-\mathcal{F}(v) \leq-d \mathcal{F}(u)[v-u]=(\mu, v-u) \leq\left\langle\mu_{+}, v-u\right\rangle,
$$

which concludes the proof.

Within this setting, the parabolic problem (2.8) would read

$$
\left\{\begin{array}{l}
\dot{u}(t)=-\partial_{+}^{\circ} \mathcal{F}(u(t)) \quad \text { in } L^{2} \text { for a.e. } t \in(0, T) \\
u(0)=u_{0} .
\end{array}\right.
$$

Clearly, the non-uniqueness issue for the parabolic problem (2.8), applies also here. Moreover, we have the following.

Remark B.3. The unilateral subdifferentials $\partial_{+}^{\circ} \mathcal{F}(u)$ and $\partial_{+} \mathcal{F}(u)$ are not monotone in $L^{2}$, as the following example shows.

For $f=-1$ let $\mathcal{F}(u)=\frac{1}{2} \int_{(-1,1)}\left|u^{\prime}\right|^{2}+u d x$ and $u_{0}=0$. Clearly $\mathcal{F}(u) \geq$ $\mathcal{F}\left(u_{0}\right)$ for every $u \geq u_{0}$, hence $0=\xi_{0} \in \partial_{+} \mathcal{F}\left(u_{0}\right)$. Clearly $\xi_{0} \in \partial_{+}^{\circ} \mathcal{F}\left(u_{0}\right)$. On other hand, let $u \in H_{0}^{1}$ with $u>0$ in $(-1,1)$ and $\left[u^{\prime \prime}-1\right]_{+} \not \equiv 0$. Then, by 
Lemma B.2 we have $\partial_{+}^{\circ} \mathcal{F}(u)=\left\{-\left[u^{\prime \prime}-1\right]_{+}\right\}$. Denoting $\xi_{u}=-\left[u^{\prime \prime}-1\right]_{+}$we have

$$
\left\langle\xi_{u}-\xi_{0}, u-u_{0}\right\rangle=-\left\langle\left[u^{\prime \prime}-1\right]_{+}, u\right\rangle<0
$$

and thus $\partial_{+}^{\circ} \mathcal{F}(u)$ is not monotone. As a consequence, $\partial_{+} \mathcal{F}(u)$ is not monotone, since it is larger than $\partial_{+}^{\circ} \mathcal{F}(u)$.

\section{Appendix C. Unconstrained gradient flow}

The aim of this last section is to provide, to the unfamiliar reader, the equivalence between gradient flows and curves of maximal slope (for our family of functionals) in the case of unconstrained evolutions. As we have already observed, for unilateral evolutions these notions are not always equivalent.

If $A u \in L^{2}$ then the $L^{2}$-gradient of the energy $\mathcal{E}$ reads

$$
\langle\nabla \mathcal{E}(u), v\rangle=d \mathcal{E}(u)[v]=-(A u, v) .
$$

In a similar way, $\nabla \mathcal{F}(t, u)=-A u-f(t)$.

Proposition C.1. If $u \in H^{1}\left(0, T ; L^{2}\right)$ and $A u \in L^{2}\left(0, T ; L^{2}\right)$ then $\dot{u}(t)=$ $-\nabla \mathcal{F}(t, u(t))$ if and only if

$$
\dot{\mathcal{E}}(u(t)) \leq-\frac{1}{2}\|\dot{u}(t)\|_{L^{2}}^{2}-\frac{1}{2}\|\nabla \mathcal{F}(t, u(t))\|_{L^{2}}^{2}+\langle f(t), \dot{u}(t)\rangle
$$

a.e. in $(0, T)$.

Proof. Under the regularity assumptions on $u$, we know by [10, Lemma 3.3] that the energy $t \mapsto \mathcal{E}(u(t))$ is absolutely continuous and that

$$
\dot{\mathcal{E}}(u(t))=\langle\nabla \mathcal{E}(u(t)), \dot{u}(t)\rangle=\langle\nabla \mathcal{F}(t, u(t)), \dot{u}(t)\rangle+\langle f(t), \dot{u}(t)\rangle
$$

a.e. in $(0, T)$. If $\dot{u}(t)=-\nabla \mathcal{F}(t, u(t))$ then

$$
\begin{aligned}
\langle\nabla \mathcal{F}(t, u(t)), \dot{u}(t)\rangle & =-\|\nabla \mathcal{F}(t, u(t))\|_{L^{2}}^{2}=-\|\dot{u}(t)\|_{L^{2}}^{2} \\
& =-\frac{1}{2}\|\dot{u}(t)\|_{L^{2}}^{2}-\frac{1}{2}\|\nabla \mathcal{F}(t, u(t))\|_{L^{2}}^{2}
\end{aligned}
$$

and (C.2) reads

$$
\dot{\mathcal{E}}(u(t))=-\frac{1}{2}\|\dot{u}(t)\|_{L^{2}}^{2}-\frac{1}{2}\|\nabla \mathcal{F}(t, u(t))\|_{L^{2}}^{2}+\langle f(t), \dot{u}(t)\rangle .
$$

Now, assume $\dot{\mathcal{E}}(u(t)) \leq-\frac{1}{2}\|\dot{u}(t)\|_{L^{2}}^{2}-\frac{1}{2}\|\nabla \mathcal{F}(t, u(t))\|_{L^{2}}^{2}+\langle f(t), \dot{u}(t)\rangle$. By Cauchy inequality

$$
\begin{aligned}
\langle\nabla \mathcal{F}(t, u(t)), \dot{u}(t)\rangle & \geq-\|\nabla \mathcal{F}(t, u(t))\|_{L^{2}}\|\dot{u}(t)\|_{L^{2}} \\
& \geq-\frac{1}{2}\|\dot{u}(t)\|_{L^{2}}^{2}-\frac{1}{2}\|\nabla \mathcal{F}(t, u(t))\|_{L^{2}}^{2} .
\end{aligned}
$$

Hence (C.2) yields

$$
\dot{\mathcal{E}}(u(t)) \geq-\frac{1}{2}\|\dot{u}(t)\|_{L^{2}}^{2}-\frac{1}{2}\|\nabla \mathcal{F}(t, u(t))\|_{L^{2}}^{2}+\langle f(t), \dot{u}(t)\rangle
$$

and thus

$$
\dot{\mathcal{E}}(u(t))=-\frac{1}{2}\|\dot{u}(t)\|_{L^{2}}^{2}-\frac{1}{2}\|\nabla \mathcal{F}(t, u(t))\|_{L^{2}}^{2}+\langle f(t), \dot{u}(t)\rangle .
$$


As a consequence, by (C.2) the inequalities in (C.3) turn into equalities and give

$$
\langle\nabla \mathcal{F}(t, u(t)), \dot{u}(t)\rangle=-\|\nabla \mathcal{F}(t, u(t))\|_{L^{2}}\|\dot{u}(t)\|_{L^{2}} .
$$

Cauchy inequality implies $\dot{u}(t)=-\nabla \mathcal{F}(t, u(t))$.

\section{References}

[1] Akagi, G., Efendiev, M.: Allen-Cahn equation with strong irreversibility. Eur. J. Appl. Math. 30(4), 707-755 (2019)

[2] Akagi, G., Kimura, M.: Unidirectional evolution equations of diffusion type. J. Differ. Equ. 266(1), 1-43 (2019)

[3] Almi, S., Belz, S., Negri, M.: Convergence of discrete and continuous unilateral flows for Ambrosio-Tortorelli energies and application to mechanics. ESAIM Math. Model. Numer. Anal. 53(2), 659-699 (2019)

[4] Almi, S., Negri, M.: Analysis of staggered evolutions for nonlinear energies in phase field fracture. Arch. Ration. Mech. Anal. 236, 189-252 (2020)

[5] Ambrosio, L., Gigli, N., Savaré, G.: Gradient flows in metric spaces and in the space of probability measures. Lectures in Mathematics ETH Zürich. Birkhäuser, Basel (2005)

[6] Ambrosio, L., Tortorelli, V.M.: Approximation of functionals depending on jumps by elliptic functionals via $\Gamma$-convergence. Commun. Pure Appl. Math. 43(8), 999-1036 (1990)

[7] Arai, T.: On the existence of the solution for $\partial \varphi\left(u^{\prime}(t)\right)+\partial \psi(u(t)) \ni f(t)$. J. Fac. Sci. Univ. Tokyo Sect. IA Math. 26(1), 75-96 (1979)

[8] Babadjian, J.-F., Millot, V.: Unilateral gradient flow of the Ambrosio-Tortorelli functional by minimizing movements. Ann. Inst. H. Poincaré Anal. Non Linéaire 31(4), 779-822 (2014)

[9] Barbu, V.: Nonlinear Semigroups and Differential Equations in Banach Spaces. Noorhoff (1976)

[10] Brézis, H.: Opérateurs maximaux monotones et semi-groupes de contractions dans les espaces de Hilbert. North-Holland Publishing Co., Amsterdam (1973)

[11] Crismale, V., Lazzaroni, G.: Viscous approximation of quasistatic evolutions for a coupled elastoplastic-damage model. Calc. Var. Partial Differ. Equ. 55(1), 54 (2016). (Art. 17)

[12] Dal Maso, G., Francfort, G.A., Toader, R.: Quasistatic crack growth in nonlinear elasticity. Arch. Ration. Mech. Anal. 176(2), 165-225 (2005)

[13] De Giorgi, E., Marino, A., Tosques, M.: Problemi di evoluzione in spazi metrici e curve di massima pendenza. Atti Accad. Naz. Lincei Rend. Cl. Sci. Fis. Mat. Natur. 68(3), 180-187 (1980) 
[14] De Giorgi, E.: New problems on minimizing movements. In: Boundary Value Problems for Partial Differential Equations and Applications. Baiocchi, C., Lions, J.L. (eds.), Masson, Paris, 81-98 (1993)

[15] Evans, L.C.: Partial differential equations. American Mathematical Society, Providence, RI (1998)

[16] Gasiński, L., Papageorgiou, N.S.: Nonlinear analysis. Series in Mathematical Analysis and Applications, vol. 9. Chapman \& Hall/CRC, Boca Raton, FL (2006)

[17] Gerasimov, T., De Lorenzis, L.: On penalization in variational phase-field models of brittle fracture. Comput. Methods Appl. Mech. Eng. 354, 990-1026 (2019)

[18] Gianazza, U., Savaré, G.: Some results on minimizing movements. Rend. Accad. Naz. Sci. XL Mem. Mat. 18, 57-80 (1994)

[19] Gigli, N.: On the heat flow on metric measure spaces: existence, uniqueness and stability. Calc. Var. Partial Differ. Equ. 39(1-2), 101-120 (2010)

[20] Knees, D.: Convergence analysis of time-discretisation schemes for rateindependent systems. ESAIM Control Optim. Calc. Var. 25, 38 (2019). (Paper No. 65)

[21] Knees, D., Rossi, R., Zanini, C.: A vanishing viscosity approach to a rateindependent damage model. Math. Models Methods Appl. Sci. 23(4), 565-616 (2013)

[22] Negri, M.: A unilateral $L^{2}$-gradient flow and its quasi-static limit in phase-field fracture by alternate minimization. Adv. Calc. Var. 12(1), 1-29 (2019)

[23] Sandier, E., Serfaty, S.: Gamma-convergence of gradient flows with applications to Ginzburg-Landau. Commun. Pure Appl. Math. 57(12), 1627-1672 (2004)

[24] Santambrogio, F.: Euclidean, metric, and Wasserstein gradient flows: an overview. Bull. Math. Sci. 7(1), 87-154 (2017)

[25] Shirakawa, K., Kimura, M.: Stability analysis for Allen-Cahn type equation associated with the total variation energy. Nonlinear Anal. 60(2), 257-282 (2005)

[26] Takaishi, T., Kimura, M.: Phase field model for mode III crack growth in two dimensional elasticity. Kybernetika 45(4), 605-614 (2009)

Masato Kimura

Faculty of Mathematics and Physics

Kanazawa University

Kanazawa 920-1192

Japan

e-mail: mkimura@se.kanazawa-u.ac.jp 
Matteo Negri

Department of Mathematics

University of Pavia

Via A. Ferrata 1

27100 Pavia

Italy

e-mail: matteo.negri@unipv.it

Received: 22 March 2021.

Accepted: 6 August 2021. 